\title{
Exercise as a Treatment for Conduit Artery Dysfunction in a Comorbid State of Metabolic Syndrome and Depressive-like Symptoms
}

Chris Skinner

Follow this and additional works at: https://researchrepository.wvu.edu/etd

\section{Recommended Citation}

Skinner, Chris, "Exercise as a Treatment for Conduit Artery Dysfunction in a Comorbid State of Metabolic Syndrome and Depressive-like Symptoms" (2016). Graduate Theses, Dissertations, and Problem Reports. 6655.

https://researchrepository.wvu.edu/etd/6655

This Thesis is protected by copyright and/or related rights. It has been brought to you by the The Research Repository @WVU with permission from the rights-holder(s). You are free to use this Thesis in any way that is permitted by the copyright and related rights legislation that applies to your use. For other uses you must obtain permission from the rights-holder(s) directly, unless additional rights are indicated by a Creative Commons license in the record and/ or on the work itself. This Thesis has been accepted for inclusion in WVU Graduate Theses, Dissertations, and Problem Reports collection by an authorized administrator of The Research Repository @ WVU. For more information, please contact researchrepository@mail.wvu.edu. 


\title{
Exercise as a Treatment for Conduit Artery Dysfunction in a Comorbid State of Metabolic Syndrome and Depressive-like Symptoms
}

\section{Chris Skinner}

Thesis submitted to the School of Medicine at West Virginia University in partial fulfillment of the requirements for the degree of Masters of Science in the Department of Exercise Physiology.

\author{
Paul Chantler, Ph.D., Chair \\ Randall Bryner, Ed.D. \\ I. Mark Olfert, Ph.D. \\ Department of Exercise Physiology \\ Morgantown, West Virginia \\ 2016
}

Keywords: Cardiovascular Diseases (CVD), Metabolic Syndrome (MetS), Unpredictable Chronic Stress Model (UCMS), Inflammation, Functional Remodeling

Copyright 2016 Roy Christopher Skinner 


\section{Abstract \\ Exercise as a Treatment for Conduit Artery Dysfunction in a Comorbid State of Metabolic Syndrome and Depressive-like Symptoms}

\section{R. Christopher Skinner}

Metabolic Syndrome (Mets) is a clustering of cardiovascular risk factors that results in a three-fold increase in cardiovascular disease. MetS is known to be associated with adverse arterial remodeling, endothelial dysfunction, decreased nitric oxide (NO) bioavailability, increased inflammation, and conduit artery dysfunction. Depression/chronic stress is emerging as a potent contributor to MetS, with MetS also aiding in the progression of depression, and thus increased vascular dysfunction. These bi-directional comorbidities are continually increasing in prevalence, justifying a need for research on their interplay and potential treatments. Aerobic exercise is a widely accepted therapy for the risk factors associated with MetS, mental stress, and vascular dysfunction. However, no study has evaluated the ability of aerobic exercise to combat the vascular dysfunction caused by MetS and depressive-like symptoms. Our objective is to evaluate the effects of MetS, chronic unpredictable stress, and exercise on the conduit arteries. The central hypothesis is aerobic exercise will attenuate the harmful arterial remodeling, endothelial dysfunction, and decreased NO bioavailability caused by MetS and chronic stress. 
Table of Contents

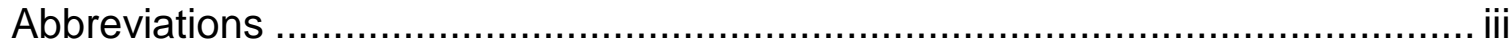

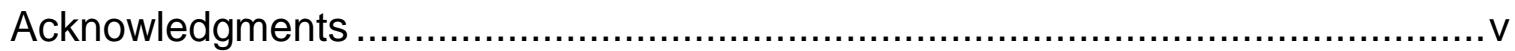

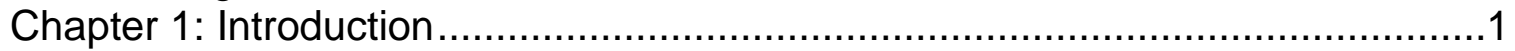

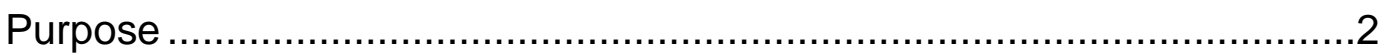

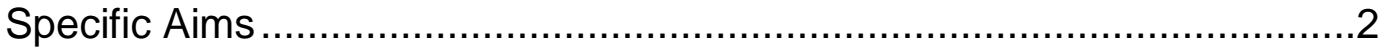

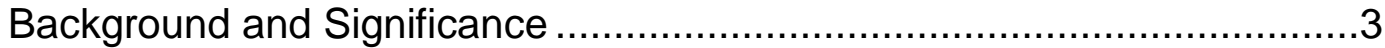

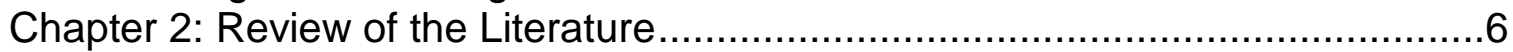

Background Information ............................................................

Defining Metabolic Syndrome ................................................

Inflammation in MetS .......................................................

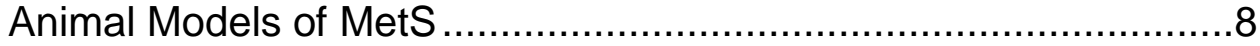

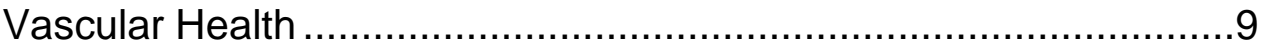

Normal Cardiovascular Function ..................................................

Diseased Cardiovascular Function..........................................10

Endothelial Function Measurements .....................................11

Diagnosing and Modeling Depression ....................................11

The Unpredictable Chronic Mild Stress Model ...........................12

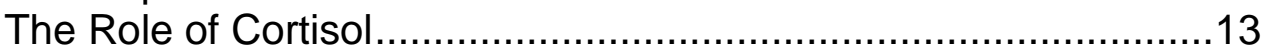

Metabolic Syndrome and Vascular Dysfunction ...................................15

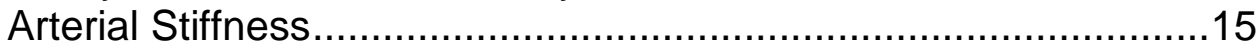

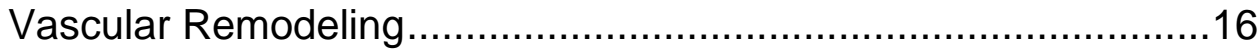

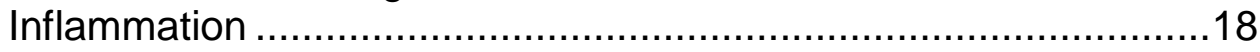

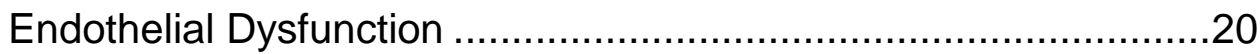

Depression Linked with MetS and Vascular Dysfunction........................21

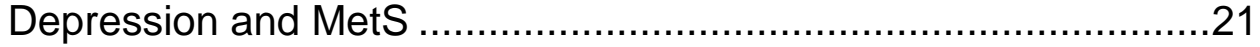

Depression and Endothelial Dysfunction ..................................21

Depression and Arterial Stiffness.........................................22

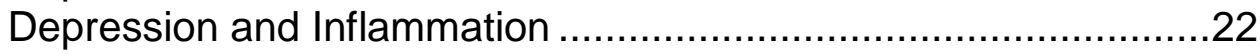

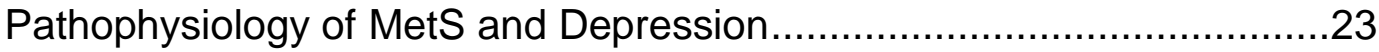

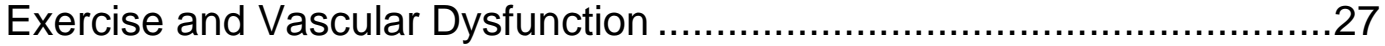

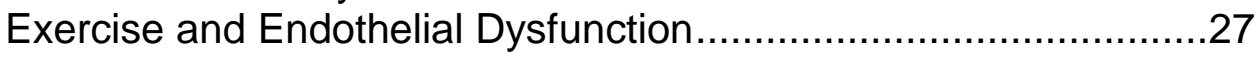

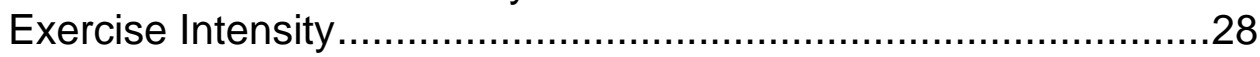

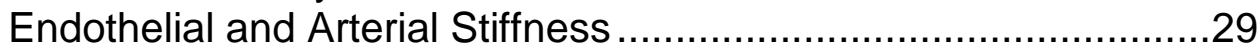

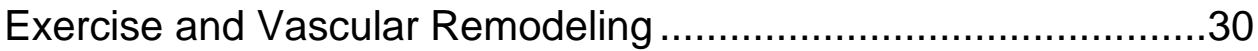

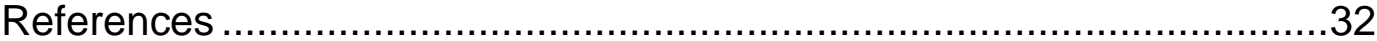

Chapter 3: Aerobic Exercise Intervention Improves Structural Carotid Artery Remodeling in a Rodent Model of Metabolic Syndrome and Chronic Stress

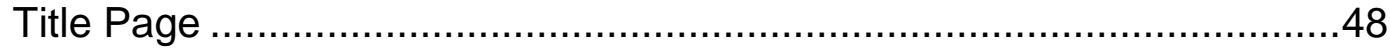

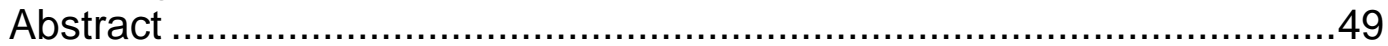

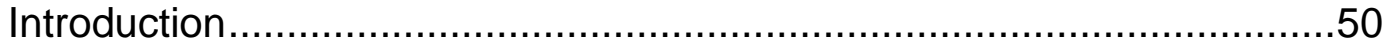

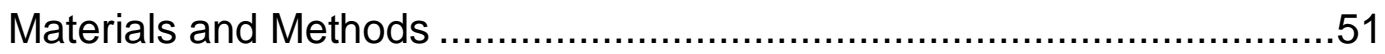

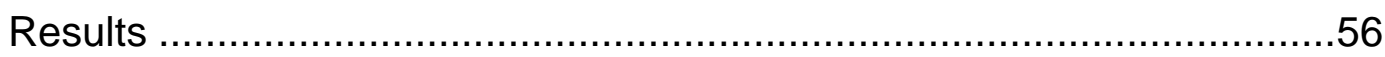

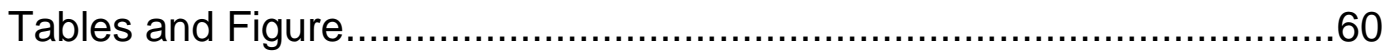

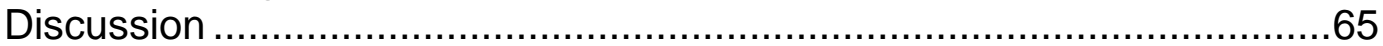


Chapter 4: Aerobic Exercise Intervention Improves Aortic Nitric Oxide-Dependent Processes in a Rodent Model of Metabolic Syndrome and Chronic Stress

Title Page ................................................................................. 71

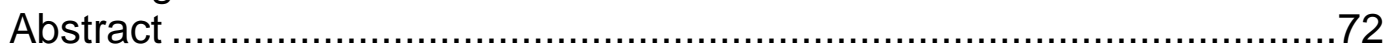

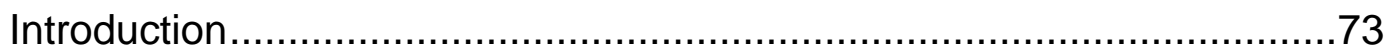

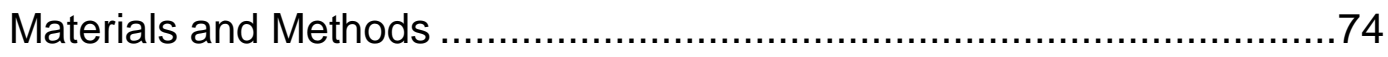

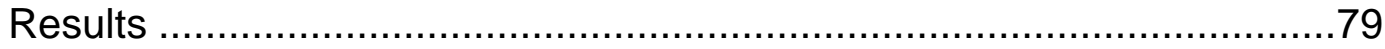

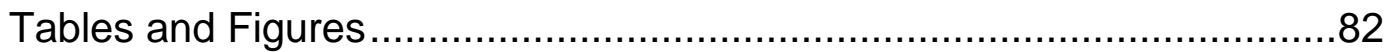

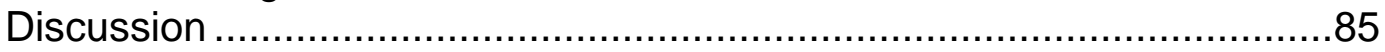

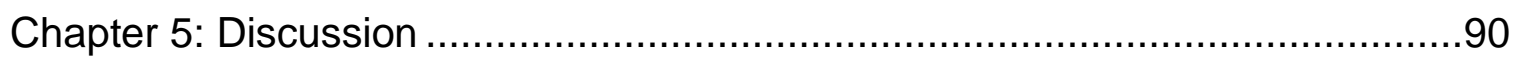

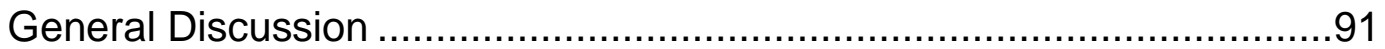

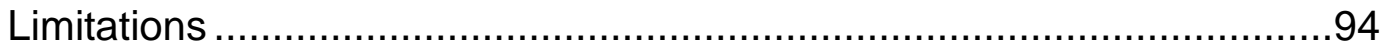

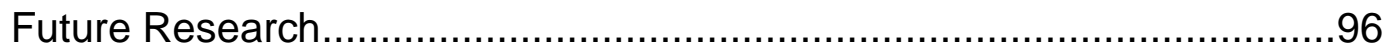

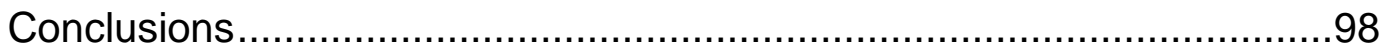

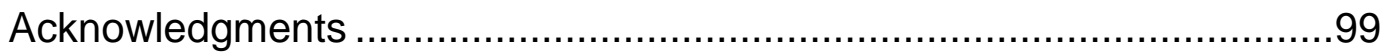

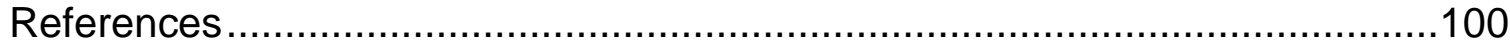




\section{Abbreviations}

Advanced Glycation End Products (AGEs)

American Association of Clinical Endocrinologists (AACE)

Angiotensin-II (ANG-II)

Aortic Pulse Pressure (APP)

Arterial Stiffness (AS)

C-Reactive Protein (CRP)

Cardiovascular (CV)

Cardiovascular Disease (CVD)

Diagnostic and Statistical Manual of Health Disorders (DSM)

Extracellular Matric (ECM)

Endothelial Nitric Oxide Synthase (eNOS)

Hypothalamus-Pituitary-Adrenal (HPA)

Inducible Nitric Oxide synthase (iNOS)

Interferon- $\mathrm{Y}$ (IFN- $\mathrm{\gamma})$

Interleukin-6 (IL-6)

Lean Zucker Rat (LZR)

Metabolic Syndrome (MetS)

National Cholesterol Treatment Panel III (NCEP:ATP III)

Matrix Metalloproteinases (MMP)

Nitric Oxide (NO)

Nitric Oxide Synthase (NOS)

Obese Zucker Rat (OZR)

Pulse Wave Velocity (PWV)

Receptor for Advanced Glycation End Products (RAGEs)

Sympathetic Nervous System (SNS)

Tissue Inhibitor of Metalloproteinase (TIMP)

Tumor Necrosis Factor- $\alpha$ (TNF- $\alpha$ ) 
Type 2 Diabetes Mellitus (T2DM)

Unpredictable Chronic Mild Stress (UCMS)

Vascular Cell Adhesion Molecule-1 (VCAM-1)

Waist:Hip Ratio (W:HR)

World Health Organization (WHO) 


\section{Acknowledgements}

I would like to thank the Department of Exercise Physiology at WVU, as everyone I have come into contact with has been helpful and friendly. Specifically, I want to thank Dr. Steve Alway for helping me transfer into exercise physiology, helping me find a lab, and aiding me in my search for graduate assistantship. I would like to thank the WVU Medicine Wellness Program for financially supporting me and always being supportive and understanding. I would like to thank my former professor's at West Virginia Wesleyan College for providing me with an excellent undergraduate education, specifically Dr. Greg Popovich. I would like to thank my labmates for always being helpful and patient with me, continuously helping me with lab work, school, and my personal life. I would like to thank my committee members, Dr. Randy Bryner and Dr. Mark Olfert, for providing me with critical insights and guidance during my time at WVU and through writing my thesis. I would like to thank Dr. Paul Chantler for accepting me into his lab, helping me find my project, and most importantly aiding me in discovering my passion for science and helping others. I am very thankful I was able to join your lab and be a part of some incredible research.

Lastly, I would like to thank my family and friends for always being supportive and encouraging during the good and bad times, the stress, and the triumph. To my parents, you are the greatest support system I will ever have. I love you both very much. 
Chapter 1:

\section{Introduction}




\section{Purpose}

Heart disease is the leading cause of death in the United States, with 169.8 of every 100,000 deaths being attributed to cardiovascular health complications (Kochanek, et al., 2014). Metabolic Syndrome (MetS) is clustering of cardiovascular risk factors highly associated with cardiovascular disease (CVD) (Grundy, et al., 2004). The estimated direct and indirect cost of cardiovascular disease is $\$ 320.1$ billion annually, with projections for the next 15 years topping $\$ 900$ billion (Mozaffarian, et al., 2015). Additionally, an estimated $18.1 \%$ of the population suffers from anxiety (Kessler, et al., 2005), with $14.4 \%$ of Americans suffering from major depressive disorder (Kessler, et al., 2012), and annual costs totaling $\$ 53$ billion (Wang, et al., 2003). Depression and MetS are intimately linked to each other, as well as cardiovascular disease (Skilton, et al., 2007). Aerobic exercise is an accepted and common treatment for both MetS and depression (Katzmarzyk, et al., 2003 \& Strohle, et al., 2009). However, no study has studied the effect of exercise on individuals simultaneously suffering from MetS and depression.

The purpose of this thesis is to determine the effects of exercise on structural vascular remodeling, nitric oxide bioavailability, and inflammation of the carotid artery and aorta in a rodent model MetS and depression as comorbidities.

\section{Specific Aims \& Hypothesis}

Specific Aim \#1: Determine the effects of 8 weeks of exercise training on structural arterial remodeling and reactivity in rodents with MetS. 
- Hypothesis \#1: Rats with MetS who exercise for 8 weeks will exhibit improved structural arterial remodeling and reactivity compared to sedentary, healthy control rodents.

Specific Aim \#2: Determine the effects of 8 weeks of chronic unpredictable stress on structural arterial remodeling and reactivity in rodents with MetS.

- Hypothesis \#2: Rodents with depressive-like symptoms and MetS will exhibit deleterious structural arterial remodeling and reactivity compared to sedentary, healthy control rodents.

Specific Aim \#3: Determine the effects of 8 weeks of exercise training on structural arterial remodeling and reactivity in rodents with MetS and depressive-like symptoms.

- Hypothesis \#3: Rats with MetS and depressive-like symptoms who exercise for 8 weeks will have structural arterial remodeling and reactivity similar to sedentary, healthy control rodents.

\section{Background and Significance}

Cardiovascular disease (CVD) is the leading cause of death worldwide, with one in every three deaths attributed to CVD (Go, et al., 2014). A plethora of lifestyle, environmental, genetic, and physiological factors can increase the prevalence of CVD. Poor diet and a sedentary lifestyle can result in negative cardiovascular risk factors, such as abdominal obesity, dyslipidemia, elevated blood pressure, and insulin resistance. This cluster of CV risk factors is known as the Metabolic Syndrome (MetS). An estimated one-quarter of the United States' population has MetS, with prevalence continuing to rise with the "obesity epidemic (Beltran-Sanchez, et al., 2013)."

In addition to MetS other environmental and psychological factors can increase the risk for negative cardiovascular outcomes. Chronic stress and depression have been linked to MetS (Koponen, et al., 2008), as well as increased risk for CVD and vascular dysfunction (Golbidi, et al., 2015). Nearly $17 \%$ of the U.S. population will suffer from 
depression over the lifespan. Due to the high prevalence of both MetS and depression, and their association with CVD, a comorbidity exists between the two afflictions (Kessler, et al., 2005).

A common hallmark of $\mathrm{CV}$ risk factors is increased arterial stiffening (AS), which can be identified by changes to the vascular wall's scaffolding proteins: collagen and elastin (Aronson, et al., 2003). The end result of MetS and depression is an increase in the amount of collagen, the tensile protein, with a decrease in elastin (Zieman, et al., 2005). In addition to altering the structural proteins of blood vessels, CVD is also linked to deviations in the production and bioavailability of nitric oxide (NO). NO regulates basal vessel tone, plays a pivotal role in vasodilation, and is associated with anti-tumor and anti-pathogenic processes (Dinerman, et al., 1993). Adverse changes to the structure and function of blood vessels is associated with increased incidence of myocardial infarction, stroke, coronary heart disease, and hypertension, leading to an increase chance of mortality (Isomaa, et al., 2001).

Exercise is a widely accepted and recommended treatment to combat CVD and to promote overall health. Studies have shown exercise to be beneficial for treating, preventing, and reversing the signs of MetS risk factors, in addition to a variety of other positive health outcomes (Katzmarzyk, et al., 2003). Exercise has also been cited as a treatment for cardiovascular ailments associated with depression (Dimeo, et al., 2001). However, exercise as a treatment for vascular dysfunction caused by the comorbid state of MetS and depression has not been adequately studied. 
Page left intentionally blank 


\section{Chapter 2:}

\section{Review of the Literature}




\section{Background Information}

\section{Defining Metabolic Syndrome}

Several definitions of MetS exist, causing confusion in diagnosis, and discrepancies in the literature (Zieman, et al., 2005). The World Health Organization (WHO) states the presence of insulin resistance and impaired glucose tolerance as essential components of MetS along with the presence of at least two other parameters; including elevated blood pressure ( $\geq 140 \mathrm{mmHg}$ systole and/or $90 \mathrm{mmHg}$ diastole), hypertriglyceridemia and/or low-HDL cholesterol ( $<35 \mathrm{mg} / \mathrm{dL}$ in men, $<39 \mathrm{mg} / \mathrm{dL}$ in women), obesity, and hyperinsulinemia (Alberti, et al., 1998; Balkau, et al., 1999). The National Cholesterol Treatment Panel III (NCEP:ATP III) has a slightly different definition of MetS. Increased waist circumference, blood lipids ( $\leq 40 \mathrm{mg} / \mathrm{dL}$ men, $50 \mathrm{mg} / \mathrm{dL}$ women), blood pressure ( $\geq$ $130 / 85 \mathrm{mmHg}$ ), and fasting glucose are all included, but insulin resistance is not considered necessary for diagnosis (Alberti, et al., 2005). The third and final major classification of MetS is from the American Association of Clinical Endocrinologists (AACE), which combines criteria from the WHO and ATP III. The AACE does not require a minimum number of risk factors, but relies on clinical judgment for diagnosis. Increased abdominal adiposity, dyslipidemia (same as NCEP: ATP III), increased blood pressure (same as NCEP: ATP III), impaired glucose tolerance, and insulin resistance are all considered risk factors for MetS (Einhorn, 2003).

\section{Inflammation in MetS}

Although not included in the definition of MetS, evidence supports the presence of chronic, low-grade inflammation in MetS, and as a risk factor for CVD (Haffner, 2006). Obesity has been linked to increased plasma concentrations of the inflammatory markers, 
tumor necrosis factor- $\alpha$ (TNF- $\alpha$ ), interleukin-6 (IL-6), and C-reactive protein (CRP) (Kern, et al., 2001; Vozarova, et al., 2001; Pradhan, et al., 2001). The inflammation associated with MetS has further been linked to cognitive impairment (Yaffe, et al., 2004), which has also been associated with depression (Kauhanen, et al., 1999). Research has found inflammation to be a predictive marker of the development of type 2 diabetes mellitus (T2DM) and obesity (Esser, et al., 2014). The well-known link between obesity and a proinflammatory state has been hypothesized as a pathophysiological mechanism explaining the onset of MetS (Emanuela, et al., 2011), and inflammation has been cited as a key component to deciphering the pathogenesis of MetS (Fuentes, et al., 2013).

\section{Animal Models of MetS}

The use of human subjects is not always possible in research, due to the need for invasive studies and ethical considerations (Zaragoza, et al., 2011). Primates and swine provide larger vessels and organs, with similar physiology to humans. However, these models are not cost efficient and are difficult to house (Russell, et al., 2006). A commonly used model for studying the cardiovascular system and its diseases is rats (Hasenfuss, 1998). Rats share similar physiology to humans, however they are typically resistant to atherosclerosis. However, several strains of rats have been developed, making certain strains suitable for CVD research. The obese Zucker rat (OZR) exhibits rapid weight gain, insulin resistance, and dyslipidemia, making it a suitable model for MetS. The OZR develops MetS-like symptoms due to an alteration to the leptin receptor, making a 10-fold reduction in the binding affinity of leptin. As a result, these leptin-deficient rats rapidly gain weight and do not gain satiety, resulting in mass food consumption (Russell, et al., 2006). 
The OZR typically becomes noticeably obese between the third and fifth week of life, and displays glucose intolerance and hyperinsulinemia around the same time. Additionally, OZRs have been shown to display endothelial dysfunction and increased markers of inflammation, further validating the OZR as an excellent model of MetS (Artinano \& Castro, 2009).

\section{Vascular Health}

\section{Normal Cardiovascular Function}

The primary function of the heart is to eject oxygenated blood into systemic circulation, and to receive deoxygenated blood from venous return (Waktare, 2002). During systole the heart contracts, ejecting blood out of the heart and into circulation. The left ventricle pumps blood into the aorta and carotid arteries, or conduit arteries. Blood then flows into smaller arteries, followed by arterioles and capillaries. Blood moves from areas of high to low pressure, with pressure decreasing in smaller vessels (Lakatta, 1990). The conduit, or elastic arteries, are essential for blood flow, as they act as a pressure reservoir, ensuring normal blood flow during diastole, or when the heart is relaxing (Joannides, et al., 1995).

The diameter of blood vessels is also essential to cardiac function. Nitric oxide (NO) plays a pivotal role in establishing basal tone of blood vessels. NO is produced by the amino acid L-arginine by the enzymatic reaction of nitric oxide synthase (NOS). In the endothelium, two forms of NOS are present; endothelial NOS (eNOS), which calciumdependent, and inducible (iNOS), or calcium-independent. Under normal conditions NO 
is constantly being produced by eNOS. Increases in blood flow, resulting in shear stress within the vessel, result in eNOS production and activity. Additionally, binding to endothelial receptors by vasodilators, such as acetylcholine, also result in NO production. NO production can also be the result of inflammation, which stimulates iNOS activity and subsequent NO release (Lancaster, 1997).

\section{Diseased Cardiovascular and Arterial Function}

The cardiovascular system undergoes several functional and structural changes in diseased states. The risk factors associated with MetS can result in decreased compliance and elasticity in the conduit arteries, resulting in a reduction in the conduits ability to supply steady blood flow during diastole and a stiffening of the arteries. In diseased states, blood vessel walls become thicker, resulting in increased lumen, reducing resistance to flow. As a result the left ventricle must work harder to pump blood to the rest of the body.

Diseases of the heart also have a profound impact on NO. In cardiovascular disease a shift is observed in terms of typical production of NO. A diseased state results in an increase in NO production from iNOS, indicating more inflammation in the vasculature. Additionally, a decrease in NO bioavailability is observed, resulting in alterations in basal tone and ability to vasodilate. 


\section{Endothelial Function Measurements}

Other molecular markers can also be used to determine vascular health. NO bioavailability and NO activity play a key role in endothelial dysfunction (Cai, et al., 2000). NO bioavailability and markers for vessel health are difficult to directly measure in humans, providing rationale for using animal models. Pressurized vessel and microvessel systems can be used to measure endothelial, smooth muscle, and nitric oxide function. In brief, vessels are removed, kept in a physiological state, exposed to vasoactive agents, and vessel diameter is measured, via video microscopy, to determine vasoreactivity (Phillips, et al., 2005).

\section{Diagnosing and Modeling Depression}

In humans, depression is diagnosed by a medical doctor, using the criteria of the latest Diagnostic and Statistical Manual of Mental Disorders (DSM) (Kessler, et al., 2005). Identification is difficult, as patients often exhibit different symptoms, such as decreased appetite and sexual desire, disrupted sleep patterns, and suicidal thoughts. Through observations and interviews doctors are able to diagnose and treat depressive disorders to the best of their ability (Harrington, 2001). Antidepressants are commonly prescribed for treatment of depression. However, these drugs have been linked to increased incidences of CVD (Hamer, et al., 2011), further highlighting a critical need for a viable treatment for depression and CVD. 


\section{The Unpredictable Chronic Mild Stress Model}

The unpredictable chronic mild stress (UCMS) protocol was developed to induce depression-like sumptoms in animals, in accordance with the DSM criteria. The UCMS protocol consists of several stressors which are implemented in a fashion so the animal cannot predict which stress will occur next. Stressors include reversing light/dark cycles, wet bedding, removing bedding, a 45 degree cage tilt, placing animals in the cage of another animal, and the removal of bedding with the addition of shallow water to a cage (Demirtas, et al., 2014).

The UCMS protocol focuses on anhedonia, or the inability to feel pleasure, by decreasing responsiveness to awards. Anhedonia is shown in rodents exposed to UCMS, as they display a decrease in sucrose-sweetened drinking water and avoidance of other natural and drug rewards (Willner, 1997). Additionally, studies have shown UCMS to decrease sexual behavior, aggression (D'Aquilia, et al., 1994), and locomotor behavior (Willner, 1997). Importantly, rodents exposed to UCMS shown signs of increased activity in the hypothalamus-pituitary-adrenal (HPA) axis and adrenal hypertrophy, which is the body's major regulator of stress response. Increase HPA-axis activity suggests the rodent is undergoing an increased level of stress (Muscat and Willner, 1992). Additionally, UCMS has been linked to corticosterone hypersecretion, further verifying the UCMS protocol as capable of inducing depressive-like symptoms (Ayensu, et al., 1995). 
Depression UCMS

DSM-IV major depressive episode/melancholia

Core symptom

Markedly diminished interest/pleasure

Decreased sexual and investigative behavior. Decreased response to rewards

Other symptoms

Significant weight loss/anorexia

Insomnia or hypersomnia

Psychomotor agitation or retardation

Fatigue or loss of energy

Depression worst in the morning

Early morning awakening

\author{
Weight loss typically around 5\% \\ Disrupted sleep patterns \\ Decreased locomotor activity \\ Decreased "active waking" in EEG \\ Effects worst at start of dark phase \\ Phase advance of diurnal rhythm of locomotor activity
}

Figure 1. Symptoms associated with depression in DSM-IV and similarities in the unpredictable chronic mild stress (UCMS) model.

\title{
The Role of Cortisol
}

Cortisol is known as the "stress hormone" due to its release through the HPA-axis in response to stress. During normal function cortisol can influence blood glucose, metabolism, specifically gluconeogenesis, anti-inflammation, blood pressure, and blood vessel tone (Dickerson, et al., 2004). Cortisol secretion has been shown to have a diurnal release, with no apparent steady-state or basal level over the course of the day. However, various diseased states, including depression and MetS, as well as exercise can dramatically alter cortisol secretion and activity (Weitzman, et al., 1971).

Depressed individuals have been shown to secrete substantially more cortisol than healthy individuals (Sachar, et al., 1973; Yehuda, et al., 1996). Additionally, individuals with depression have been shown to have a blunted response to cortisol secretion, indicating a decreased ability to physiologically handle stress. Depressed individuals also experienced impaired stress recovery in correlation with this blunted response to cortisol 
(Burke, et al., 2005). Other studies show prolonged increased in cortisol to permanently downregulate hippocampal cell receptors and produce chronic inflammation in the hippocampus. These alterations are associated with diminished serotonin synthesis in the brain and altered neurotransmitter function (Stokes, 1995).

Individuals with an increased waist:hip ratio (WHR) have been shown to have an exaggerated cortisol response to stress (Epel, et al., 2000). Individuals with MetS, much like depressed individuals, have been shown to have hyperactivity of the HPA axis, resulting in chronic cortisol secretion. This increase in cortisol has been linked to increases in visceral fat accumulation, further exacerbating the situation as the WHR grows (Anagnostis, et al., 2009). Central obesity, a MetS risk factor, is positively correlated with elevated cortisol, which can then lead to insulin resistance, hypertension, and dyslipidemia, further progressing MetS. Prolonged overstimulation of cortisol and the HPA axis can result in deficiencies in stress-reducing feedback mechanisms, resulting in chronic stress (Bjorntorp, et al., 2000).

Intensity of exercise plays a large role in cortisol secretion. High intensity exercise has been shown to significantly increase serum cortisol levels, while low intensity exercise shows little effect (Hill, et al., 2008). Another study suggested exercise to stimulate an increase in the rate of cortisol uptake by peripheral tissues, with cortisol returning to normal post-exercise training (Few, 1974). 


\section{Metabolic Syndrome and Vascular Dysfunction}

\section{Arterial Stiffness}

The vascular dysfunction associated with MetS has been studied in depth. Early studies established the relationship between MetS and vascular dysfunction. One study focused on the interplay of AS, MetS, and hypertension. MetS was found to be associated with increased pulse wave velocity (PWV), with PWV being greater in those MetS and hypertension group than the hypertension alone. Aortic pulse pressure (APP) was found to be higher in both hypertensive groups, regardless of the presence of MetS. Results suggest increased arterial stiffness to be present in MetS regardless of the presence of high blood pressure (Kangas, et al., 2013). These findings further support earlier research, which concluded MetS was an independent determinant of arterial stiffness (Schillaci, et al., 2005; Ferreira, et al., 2005).

MetS and AS have also been studied with ischemic stroke included as a parameter, again measuring vascular health. Subjects with MetS and ischemic stroke were shown to have significantly higher PWV than those suffering from ischemic stroke without MetS. This study shows an association between arterial stiffness and MetS, while also indicating MetS as a link to other diseases of the cardiovascular system (Tuttolomondo, et al., 2012). These results are in agreement with other studies. One study concluded individuals with MetS had a more prevalent history of coronary heart disease, myocardial infarction, and stroke than those without MetS (Isomaa, et al., 2001). Another study found MetS to be associated with an increased risk of death from coronary heart disease and CVD, regardless of presence of type 2 diabetes mellitus (T2DM) as a risk 
factor for MetS, indicating the clustering of risk factors to increase mortality regardless of insulin resistance (Malik, et al., 2004).

\section{Vascular Remodeling}

Remodeling of the vasculature occurs as a result of diseased states, such as prolonged periods of hypertension, inflammation, T2DM, or chronic kidney disease. Vascular remodeling typically associated with MetS is hypertrophic inward, which results in a thicker wall, decreased distensibility, increase wall:lumen ratio (WLR), and an increase in cross-sectional wall area (CSWA) (van Varik, et al., 2013). Matrix metalloproteinases (MMP) have been shown to be key in remodeling in physiological and pathological states. MMPs are under the regulation of inflammation, oxidative stress, injury, and hemodynamics (Galis and Khatri, 2002). Blood flow has been shown to be a powerful regulator of MMP expression, with temporary carotid artery flow cessation in a murine model causing significant upregulations in MMP-9 (Bassiouny, et al., 1998). The NO produced by shear stress has been shown to be crucial to the remodeling process. A study utilized eNOS-null mice found a lack of compensatory remodeling to increases in shear stress (Rudic, et al., 1998). In another study, rabbit femoral arteries were shown to undergo constrictive remodeling following balloon injury, which was attributed to a decrease in NO bioavailability and an increase in collagen deposition (Lafont, et al., 1999). MMPs can also be activated by NO in diseased states. When NO and superoxide are simultaneously produced peroxynitrate is generated, which can lead to activation of MMPs and exacerbate vascular dysfunction (Rajagopalan, et al., 1996). 


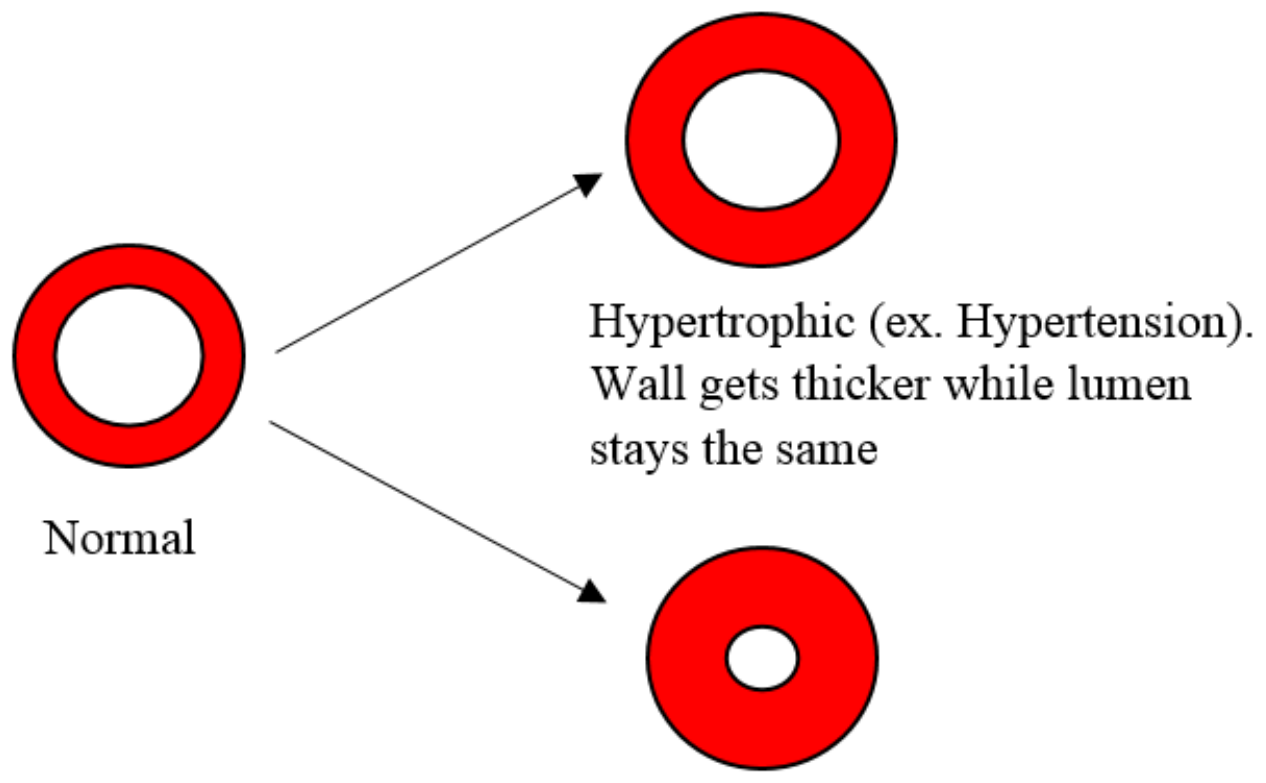

Inward hypertrophic (ex.

Large artery remodeling).

Wall gets thicker while

lumen size decreases

Figure 2. Common types of large artery remodeling in diseased states.

Decrease in eNOS has been linked to an increase in remodeling in response to arterial stress (Rudic, et al., 1998). Diseases, like MetS and depression, can result in hypertrophic remodeling on the arterial wall. Hypertrophic remodeling is the thickening of the arterial wall and can occur inward, where the wall becomes thicker inward, or outward (van Varik, et al., 2013).

MetS and T2DM have both been shown to cause the formation of advanced glycation end products (AGEs), or proteins or lipids that have become glycated following exposure to oxidative stress and copious amounts of sugar. AGEs can alter the vascular basement membrane and ECM through binding to receptors for AGEs. This binding leads 
to increases in collagen cross linking, decreased elastin, and arterial stiffness (Chantler \& Frisbee, 2014). AGEs also diminish NO activity and cause endothelial dysfunction (Goldin, et al., 2006). AGEs have been shown to be prevalent in the conduit arteries of animal models of MetS, yielding them as a translational point of research in the disease (Reddy, 2004; Hill, et al., 2001).

MetS results in chronic injurious stimuli to the ECM, which results in structural alterations, tissue dysfunction, and loss of structure and form. Collagen, the strength and tensile protein in the artery, has been shown degrade and re-proliferate at a higher rate when exposed to high levels of MMP-9, resulting in a stiffer vessel. Pathological conditions like MetS alter the normal activity of MMPs and other important remodeling factors, which has a profound impact of the health of the vasculature (Galis and Khatri, 2002).

\section{Inflammation}

Other findings elucidated the underlying inflammatory mechanisms associated with MetS and endothelial dysfunction. Individuals with MetS were found to have significantly higher circulating concentrations of all inflammatory cytokines. The inflammatory markers were correlated with E-selectin and vascular cell adhesion molecule-1 (VCAM-1), which are markers of endothelial function (Dinerman, et al., 1993). The inflammatory markers were also found to have a relationship with arterial stiffness (Dinerman, et al., 1993). These results support other findings suggesting MetS is associated with a specific profile of inflammatory cytokines (Troseid, et al., 2009; Weiss, et al., 2011). 
In contrast, Weiss, et al. found increases in arterial stiffness and endothelial dysfunction associated with increased markers of IL- 6 to be independent of the presence of MetS, indicating vascular dysfunction may be the result of inflammation and not necessarily MetS (Weiss, et al., 2013). However, other literature provides evidence a proinflammatory state induces insulin resistance, ultimately resulting in MetS. Combining the findings regarding vascular dysfunction and inflammation, and the inflammatory effect on the onset of MetS, it can be concluded vascular dysfunction is the result of the culmination of chronic inflammation and the pathogenesis of MetS (Dandona, et al., 2004).

MMPs are another marker of vascular dysfunction and inflammation. MMPs are tightly controlled by tissue inhibitor of metalloproteinase (TIMP) and an imbalance in the two can result in pathogenic processes, such as atherosclerosis (Rodriguez, et al., 2010). Several MMPs exist, each with a unique function and significance. Individuals with MetS have been shown to have higher circulating concentrations of MMP-9, yielding an increased risk for vascular dysfunction (Goncalves, et al., 2009). Another study confirmed the increase in MMP-9 in those with MetS, as well as TIMP-1 and TIMP-2, suggesting higher circulating levels of these factors could be associated with MetS (Cicero, et al., 2007). Both MMP-2 and MMP-9 were found to be increased in the plasma in individuals with heart failure (Alberti, et al., 2003). Inhibition of the MMPs has been shown to reverse hypertension and inhibit vascular dysfunction in a rat model (Castro, et al., 2007). Furthermore, NO formation and plasma MMP-9 levels have been found to have an inverse relationship, suggesting MMP-9 directly impacts NO bioavailability and vascular health (Demacq, et al., 2008). 


\section{Endothelial Dysfunction}

In addition to arterial stiffening, endothelial dysfunction is common in diseases of the cardiovascular system (Cai \& Harrison, 2000; Heitzer, et al., 2001; Hadi, et al., 2005). Many pathways converge to cause endothelial dysfunction, such as inflammation (Goldberg, 2009), aging (Csiszar, et al., 2008), and obesity (Tounian, et al., 2001). One of the key regulators of endothelial function is NO (Rees, et al., 1989; Steinberg, et al., 1994). NO plays a crucial role in endogenous vasodilation (Palmer, et al., 1988) and has been shown to be impaired in diseased states, such as diabetes (Williams, et al., 1996).

Endothelium dependent and independent vasodilation were found to be severely impaired in an animal model of MetS. The decrease in vasodilation was attributed to either impaired production of $\mathrm{NO}$ by the endothelium and/or smooth muscle, or a reduction in NO bioavailability. An increase in oxidative stress in the impaired vessels was also found (Ferreira, et al., 2005). These results are further supported as insulin resistance has been linked to endothelial dysfunction (Stuhlinger, et al., 2002). Furthermore, TNF- $\alpha$ overexpression has also been shown to impair endothelium-dependent vasodilation, additionally linking MetS and inflammation to cardiovascular complications (Picchi, et al., 2006).

Further exacerbating the vascular dysfunction caused by MetS is depression. The interaction between these two diseases and their negative effect on the vasculature results in comorbidities. 


\section{Depression linked with MetS and Vascular Dysfunction}

\section{Depression and MetS}

The link between MetS and depression has recently become more evident and researched. Rodents exposed to the UCMS model of depression experienced impaired vasodilation and endothelial dysfunction following eight weeks of stresses (Isingrini, et al., 2011). Endothelial dysfunction has been cited as a potential link between depression and CVD, with the UCMS protocol verified as a reliable depression-like model in rodents (d'Audiffret, et al., 2010).

In humans, depression is positively correlated with diabetes in middle-aged women. Incidence is attributed to an increase in central adiposity, indicating the potential role of MetS in the onset of depression (Everson-Rose, et al., 2004). Building on this, individuals with MetS were shown to have a greater incidence of depression. This association was present following adjustments for age, gender, socioeconomic status, smoking status, BMI, physical activity, and previous cardiovascular events, indicating a relationship exists between the two diseases (Skilton, et al., 2007). However, previous research found young women, but not men to have a higher association of MetS and depression (Raikkonen, et al., 2007; Kinder, et al., 2004) . Additionally, one study found no association among age, MetS, and depression (Herva, et al., 2006).

\section{Depression and Endothelial Dysfunction}

The association between MetS and depression establishes the diseases as comorbidities. Similar to MetS, depression has also been shown to be involved with vascular dysfunction (Rajagopalan, et al., 2001). Individuals with depression were shown 
to have decreased eNOS activity and NO levels, when compared to healthy controls (Chrapko, et al., 2004). Endothelial dysfunction has been proposed as a link between cardiovascular disease and depression (Sherwood, et al., 2005). Endothelial dysfunction was found to be present in individuals following treatment with antidepressants (Broadley, et al., 2002), and also during depression remission (Rybakowski, et al., 2006). Given the interaction between antidepressants and cardiovascular drugs this presents a need for different therapy in combating the onset of both diseases (Hamer, et al., 2011).

\section{Depression and Arterial Stiffness}

Depression has also been linked to arterial stiffness (Tiemeier, et al., 2003;Yeragani, et al., 2006) and anxiety (Jiang, et al., 2004). Depressed and anxious subjects were both found to have increased arterial stiffness $(p=0.01)$. Subjects with higher degrees of depression and/or anxiety, or with long-term conditions were shown to have a further increase in AS, providing evidence for a dose-response relationship between depression and arterial stiffening (Seldenrijk, et al., 2011).

\section{Depression and Inflammation}

Depression is also associated with inflammation (Stewart, et al., 2009). Inflammation has been linked to structural changes in vascular proteins and reductions in NO bioavailability. These findings suggest diseases of inflammation, such as MetS and depression, are associated with vascular dysfunction (Zieman, et al., 2005). Conflict exists regarding the role inflammation plays in the relationship between depression and CV health. Some studies suggest inflammation plays only a minor role (Vaccarino, et al., 2007). However, 
evidence indicates inflammation as a cause of depression and the reverse, as well as depression to be a cause of inflammation, resulting in a pathologic process, which increases risk for diseases of the cardiovascular system (Shimbo, et al., 2005).

The association between depression and MetS is also bidirectional. Individuals with decreased carotid artery distensibility, a common feature of MetS, were shown to be more likely to have a depressive disorder. This indicates MetS and arterial stiffness as potential risk factors and contributors to depression (Tiemeier, et al., 2003).

With the suggestion that MetS and depression have an association with each other, and to vascular dysfunction, their comorbidity is established. Aerobic exercise is widely studied and recognized treatment for vascular dysfunction caused by both MetS and depression as independent diseases, however no study has investigated the exercise treatment for both simultaneously.

\section{Pathophysiology of MetS and Depression}

The onset of MetS or depression is not an overnight phenomenon. Each disease stems from a chronic stimulus, resulting in disease onset, while also increasing the risk for the other (Dunbar, et al., 2008). Chronic stress increase HPA-axis activity, resulting the release of corticotrophin-releasing hormone $(\mathrm{CRH})$ from the hypothalamus. $\mathrm{CRH}$ stimulates the anterior pituitary gland to release adrenocorticotropic hormone (ACTH), which in turns results in cortisol release from the adrenal glands (Breznoscakova \& Nagyova., 2013). Cortisol is anti-inflammatory when functioning normally (Cline, et al., 1966). However, chronic cortisol activation and circulation results in a removal of the antiinflammatory effects of cortisol, making it a pro-inflammatory hormone (Brown, 2013). 
This chronic release of cortisol results in the release of several inflammatory cytokines, including TNF- $\alpha, \mathrm{IL}_{1}-\beta$, and interferon (IFN)- $\gamma$, into circulation and the vasculature. This increase in inflammation results in immune cell attraction and infiltration, increasing the risk of LDL oxidation and foam cell formation (Miller, et all., 2009). Additionally, the inflammatory cytokines also generate ROS, leading to an increase in superoxides and NOX, while decreasing NO and anti-oxidant defenses (Brown, 2013).

Chronic cortisol release also stimulates an increase in sympathetic nervous system (SNS) activity, which in turn increases heart rate and blood pressure (Paredes, et al., 2014). Overtime, this increase in BP results in increased wall stress and vascular remodeling (Heagerty, et al., 1993). This vascular remodeling is mediated through increases in MMPs and collagen, with smooth muscle cell hypertrophy. The culmination of this remodeling is macrovascular dysfunction (Chantler \& Frisbee, 2014). The increase in SNS activity also activates the renin-angiotensin-aldosterone system (RAAS). Increased RAAS activity stimulates an increase in angiotensin (ANG)-II activity (Majumder \& Wu, 2014). Increases in circulating ANG-II will result in increased binding to ANG-II type-I receptors (AT1R). This binding will stimulate ROS generation, inflammation, insulin inhibition, and vascular smooth muscle proliferation. Importantly, an increase NfKB signaling will result in TNF- $\alpha$ and $\mathrm{IL}_{1}-\beta$ activation, resulting in a cascade of pro-thrombotic and pro-inflammatory factors, including CRP, V-CAM, I-CAM, MMPs, and various cytokines (Prasad, et al., 2004). The end result of this pathway is endothelial dysfunction, through decreased in NO bioavailability and chronic increases in vasoconstriction.

MetS shares many of the pathophysiological mechanisms with depression. However, MetS has distinct features increasing cardiovascular risk, as well as 
exacerbating depression. Physical inactivity, smoking, stress, eating in caloric excess, and genetics are all known risk factors of MetS. Overtime individuals chronically place themselves in positive energy balance, from overeating and lack of physical activity, resulting in adipose tissue hyperplasia and hypertrophy. This results in altered free fatty acid (FFA) metabolism, leading to increased circulation of FFA, increased gluconeogenesis, and dyslipidemia. Altered FFA metabolism also results in insulin resistance, pancreatic $\beta$-cell impairment, hyperglycemia, and eventually T2DM (Kaur, 2014).

MetS also alters the release of adipocytes, specifically increasing leptin and decreasing adiponectin (Kaur, 2014). This decrease in adiponectin further exacerbates insulin resistance and progression of T2DM (Whitehead, et al., 2006). The increase in leptin results in leptin resistance, and an inability to gain satiety (Korner, et al., 2007). The combination of altered FFA metabolism and adipokine release results in stimulation of the SNS, resulting in activation of the same ANG-II pathway involved in depression. MetS also increases oxidative stress and ROS generation, leading to similar complications associated with depression, such as NOX and superoxide generation, paired with a decrease in NO bioavailability and endothelial dysfunction (Kaur, 2014). The result of these two converging diseases is chronic inflammation, increased vascular remodeling, elevated blood pressure and heart, altered insulin signaling and insulin resistance, and endothelial dysfunction, resulting in a severe increased risk for CVD and death. 


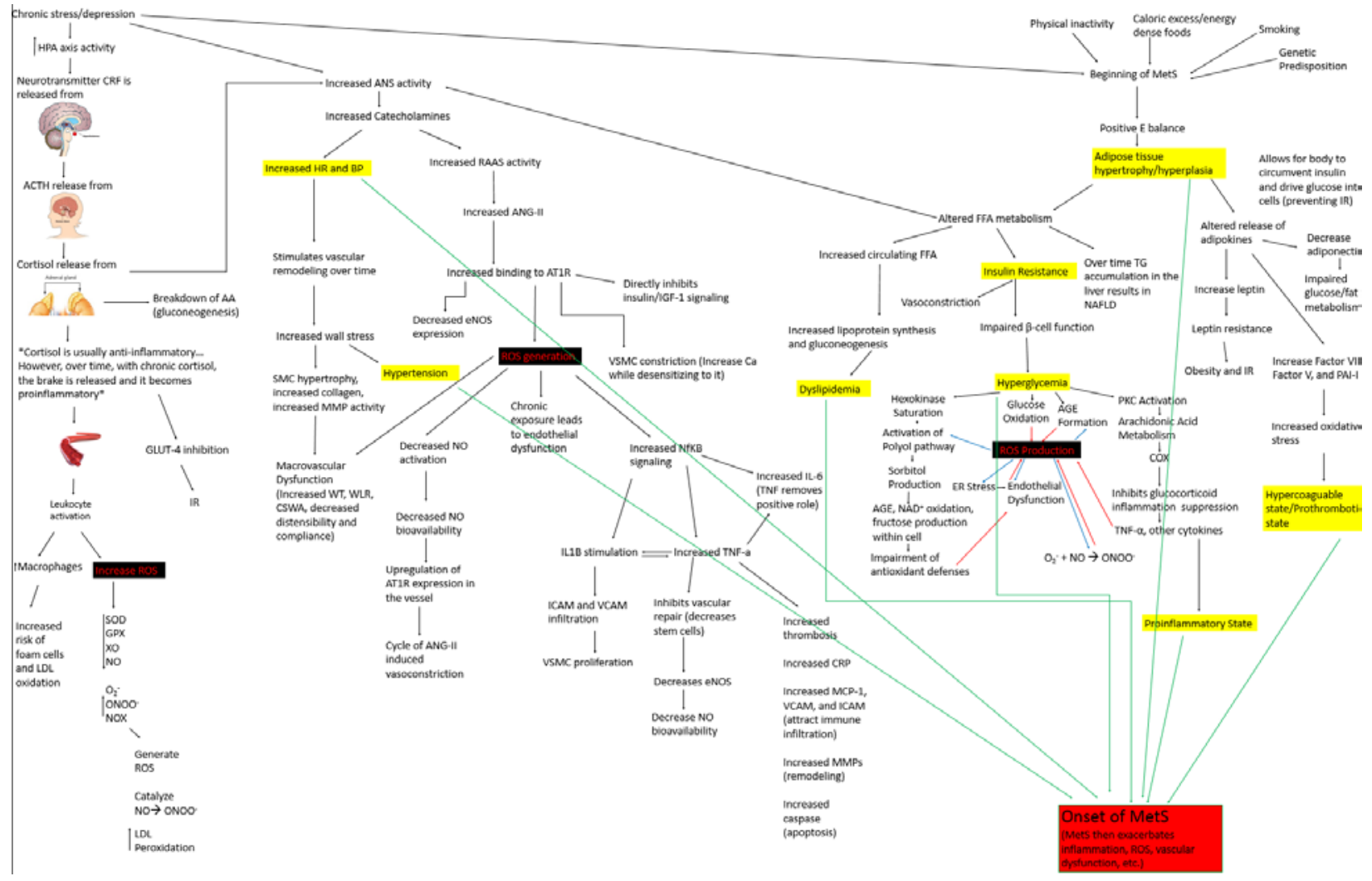

Figure 3. Pathways linking Metabolic Syndrome (MetS) and chronic stress/depression. Abbreviations: HPA, hypothalamus-pituitary-adrenal; CRF, corticotrophin releasing factor; AA, amino acids; ROS, reactive oxygen species; LDL, low-density lipoproteins; SOD, superoxide dismutase; GPX, glutathione peroxidase; NO, nitric oxide; O2-, superoxide; ONOO-, perioxynitrate, NOX, NADPH oxidase; XO, xanthine oxidase; IR, insulin resistance; ANS, autonomic nervous system; HR, heart rate; $\mathrm{BP}$, blood pressure; SMC, smooth muscle cell; MMP, matrix metalloproteinases; WT, wall thickness; WLR, wall:Iumen ratio; CSWA, cross-sectional wall area; RAAS, renin-angiotensin aldosterone system; ANG-II, angiotensin II; AT1R, angiotensin II type-I receptor; IGF-1, insulin-like growth factor-1; VSMC, vascular smooth muscle cell; Ca, calcium; NfKB, nuclear factor kappa-light-chain-enhancer of activated B cells; IL1 $\beta$, interleukin-1 $\beta$; ICAM, intracellular cell adhesion molecule; VCAM, vascular cell adhesion molecule; TNF- $\alpha$, tumor necrosis factor- $\alpha$; IL-6, interleukin-6; eNOS, endothelial nitric oxide synthase; CRP, c-reactive protein; MCP-1, monocyte chemoattractant protein-1; E, energy; FFA, free-fatty acid; TG; triglycerides; NAFLD; non-alcoholic fatty liver disease; PAI-1, plasminogen activator inhibitor-1; AGE, advanced glycation end products; $\mathrm{NAD}^{+}$, nicotinamide adenine dinucleotide; PKC, protein kinase C; COX, cyclooxygenases. 


\section{Exercise and Vascular Dysfunction}

The effects of exercise on cardiovascular and mental health have been widely investigated. Aerobic exercise training has been reported to be beneficial to CV health. Individuals with MetS participating in 20 weeks of supervised aerobic exercise saw significant improvements in cholesterol, blood pressure, glucose tolerance, and waist circumference (Katzmarzyk, et al., 2003). Chronic exercise training was able to increase extracellular NOS and NO production, as well as NO gene expression, in dogs (Sessa, et al., 1994)

In a rat model of hypertension, swimming was able to reduce degradation of elastin and improve vessel health (Andrade, et al., 2013). When rats were fed a high-fat diet to induce MetS followed by endurance training dyslipidemia, blood pressure, glucose tolerance, cholesterol, obesity, and endothelial function were improved. These improvements persisted despite the rats being maintained on a high-fat diet throughout the study. The study results showed exercise to be beneficial even in the absence of dietary intervention (Touati, et al., 2011).

\section{Exercise and Endothelial Dysfunction}

Obese Zucker rats had increased NO bioavailability following chronic treadmill running when compared to sedentary rats (Frisbee, et al., 2006). Individuals with coronary artery disease participated in a four-week exercise-training regimen, which resulted in improvement in endothelium-dependent vasodilation in epicardial and resistance vessels 
(Hambrecht, et al., 2000). These results suggest exercise training to be capable of improving MetS and CVD.

Subjects with MetS were shown to have lower markers of NO formation, indicating vascular dysfunction (Gomes, et al., 2008). Plasma and whole body nitrite, which are end products of NO release and potential storage pools for NO generation (Lundberg, et al., 2008), were decreased in individuals with MetS. Cyclic guanosine monophosphate (cGMP), a secondary messenger key to NO release in the smooth muscle, was also found to be decreased $(p<0.05)$ (Archer, et al, 1994), with oxidative stress shown to be increased $(p<0.05)$. However, exercise training was able to recover concentrations of blood nitrite and cGMP activity, while reducing oxidative stress (Gomes, et al., 2008).

\section{Exercise Intensity}

Intensity of exercise is an important factor in the treatment of vascular dysfunction. Moderate intensity exercise, defined as $40-65 \%$ of VO2 max (Johnson, et al., 2007), has been cited as beneficial in combatting MetS (Haffner, et al., 2006) and decreasing inflammation in T2DM (Zoppini, et al., 2006). These results conflict with other studies, suggesting a higher intensity of exercise is more beneficial to improving vascular dysfunction. High-intensity exercise, defined as $65-80 \%$ of VO2 max (Johnson, et al., 2007), was shown to be superior to moderate-intensity in reducing MetS risk factors (Tjonna, et al., 2008). High-intensity exercise was also more effective in reducing inflammation associated with MetS than moderate intensity exercise (Balducci, et al., 2010). However, intense exercise training caused more inflammation and a marked increase in circulating inflammation markers (Peeling, et al., 2009), suggesting moderate 
intensity exercise as a better modality for a population already suffering from chronic inflammation.

\section{Exercise and Arterial Stiffness}

Moderate intensity exercise has been shown to be beneficial in combatting vascular dysfunction in both MetS and depression. When subjects with MetS were subjected to an aerobic training regimen of moderate intensity, consisting of 8 weeks of training, they were shown to have reduced arterial stiffness (Donley, et al., 2014). Individuals with hypertension were shown to have improvements in blood pressure and arterial stiffness following four weeks of moderate intensity aerobic exercise training (Collier, et al., 2008). Short-term aerobic exercise training was shown to reduce central arterial stiffness, but have little effect on the peripheral vessels, which was determined to take a longer training regimen (Hayashi, et al., 2005). Aerobic exercise training was also determined to be beneficial in treating multifactorial arterial stiffness, caused by age, T2DM, hypertension, and high cholesterol (Madden, et al., 2009).

Similarly, when individuals with depression participated in moderate intensity walking or mindful walking protocols they were shown to have improvements in NO production and vascular function (Prakhinit, et al., 2014). Adolescents who did not partake in regular aerobic exercise were shown to have an increased risk of childhood depression, resulting in poor cardiovascular function (Waloszek, et al., 2015). Elderly individuals who had suffered from a cardiovascular incident were shown to have increased quality of life, decreased anxiety, and an increase in cognitive and cardiovascular function when subjected to an exercise training protocol as part of their cardiovascular rehabilitation 
(Fleg, et al., 2012). The ability of moderate intensity aerobic exercise to decrease vascular dysfunction in both MetS and depression, while causing minimal inflammation, makes it an ideal training protocol for combatting these comorbid states.

\section{Exercise and Vascular Remodeling}

Exercise training has been shown to have several positive outcomes in remodeling the vasculature. Exercise training results in the increase in capillarity, or angiogenesis, and the enlargement of conduit arteries, known as arteriogenesis. The increase in blood flow caused by exercise results in larger, thicker-walled elastic arteries (Tronc, et al.,1996). Chronic increases in shear stress, caused by a regular exercise program, drive increases in NO production, vasodilation, and remodeling in order to handle the increased demand placed on the vessels (Prior, et al., 2002). Critically, normal NOS function is needed for conduit remodeling to occur, as inhibition of NOS function with L-NAME was found to eliminate vascular remodeling and stunt the increase in blood flow caused by exercise training (Yang, et al., 2001).

Exercise training has been found to decrease MMP-9 expression, thereby protecting the ECM from the pathophysiological breakdown of collagen and elastin (Roberts, et al., 2006). Exercise training was also shown to increase circulating concentrations of TIMPs, which directly oppose MMP-induced ECM breakdown. Increases in TIMP further emphasize the importance of exercise training in maintaining healthy and positive vascular remodeling (Tayebjee, et al., 2005). In a model of spontaneously hypertensive rats one study found exercise training to increase elastin content in conduit arteries, with a sedentary model showing increases in collagen 
deposition. Decreases in elastin in sedentary animals were positively linked to increases in MMP-2 and MMP-9 expression. Exercise animals not only increased elastin content, but also decreased blood pressure and MMP concentrations. The authors contribute these factors to an overall decrease in hypertrophic remodeling on the exercise rats arteries (de Andrade Moraes-Teixeira, et al., 2010). 


\section{References}

Alberti, K. G. M., Zimmet, P., Shaw, J., \& IDF Epidemiology Task Force Consensus Group. (2005). The metabolic syndrome-a new worldwide definition. The Lancet, 366(9491), 1059-1062.

Alberti, Kurt George Matthew Mayer, \& Zimmet, P. f. (1998). Definition, diagnosis and classification of diabetes mellitus and its complications. part 1: Diagnosis and classification of diabetes mellitus. provisional report of a WHO consultation. Diabetic Medicine, 15(7), 539-553.

Altieri, P., Brunelli, C., Garibaldi, S., Nicolino, A., Ubaldi, S., Spallarossa, P., et al. (2003). Metalloproteinases 2 and 9 are increased in plasma of patients with heart failure. European Journal of Clinical Investigation, 33(8), 648-656.

Anagnostis, P., Athyros, V. G., Tziomalos, K., Karagiannis, A., \& Mikhailidis, D. P. (2009). The pathogenetic role of cortisol in the metabolic syndrome: A hypothesis. The Journal of Clinical Endocrinology \& Metabolism, 94(8), 2692-2701.

Andrade, G. P., Cintra, M. M., Alves, P. M., Barbosa Neto, O., Rossi e Silva, R. C., Dias da Silva, V. J., et al. (2013). Remodeling of elastic layer of aortic artery after training by swimming in spontaneously hypertensive rats. Experimental Biology and Medicine (Maywood, N.J.), 238(1), 7-11. doi:10.1258/ebm.2012.012243 [doi]

Archer, S. L., Huang, J. M., Hampl, V., Nelson, D. P., Shultz, P. J., \& Weir, E. K. (1994). Nitric oxide and cGMP cause vasorelaxation by activation of a charybdotoxinsensitive K channel by cGMP-dependent protein kinase. Proceedings of the National Academy of Sciences of the United States of America, 91(16), 7583-7587.

Aronson, D. (2003). Cross-linking of glycated collagen in the pathogenesis of arterial and myocardial stiffening of aging and diabetes. Journal of Hypertension, 21(1), 3-12.

Ayensu, W. K., Pucilowski, O., Mason, G. A., Overstreet, D. H., Rezvani, A. H., \& Janowsky, D. S. (1995). Effects of chronic mild stress on serum complement activity, saccharin preference, and corticosterone levels in flinders lines of rats. Physiology \& Behavior, 57(1), 165-169.

Balducci, S., Zanuso, S., Nicolucci, A., Fernando, F., Cavallo, S., Cardelli, P., et al. (2010). Anti-inflammatory effect of exercise training in subjects with type 2 diabetes and the metabolic syndrome is dependent on exercise modalities and independent of weight loss. Nutrition, Metabolism and Cardiovascular Diseases, 20(8), 608-617.

Balkau, B., \& Eschwege, E. (1999). Insulin resistance: An independent risk factor for cardiovascular disease? Diabetes, Obesity and Metabolism, 1(s1), 23-31. 
Bassiouny, H. S., Song, R. H., Hong, X. F., Singh, A., Kocharyan, H., \& Glagov, S. (1998). Flow regulation of $72-\mathrm{kD}$ collagenase IV (MMP-2) after experimental arterial injury. Circulation, 98(2), 157-163.

Beltrán-Sánchez, H., Harhay, M. O., Harhay, M. M., \& McElligott, S. (2013). Prevalence and trends of metabolic syndrome in the adult US population, 1999-2010. Journal of the American College of Cardiology, 62(8), 697-703.

Björntorp, P., \& Rosmond, R. (2000). The metabolic syndrome-a neuroendocrine disorder? British Journal of Nutrition, 83(S1), S49-S57.

Breznoščáková, D., \& Nagyová, I. (2013). Depression and glucose metabolism (diabetes mellitus). INTECH Open Access Publisher.

Broadley, A. J., Korszun, A., Jones, C. J., \& Frenneaux, M. P. (2002). Arterial endothelial function is impaired in treated depression. Heart (British Cardiac Society), 88(5), 521523.

Brown, N. J. (2013). Contribution of aldosterone to cardiovascular and renal inflammation and fibrosis. Nature Reviews Nephrology, 9(8), 459-469.

Burke, H. M., Davis, M. C., Otte, C., \& Mohr, D. C. (2005). Depression and cortisol responses to psychological stress: A meta-analysis. Psychoneuroendocrinology, 30(9), 846-856.

Cacicedo, J. M., Gauthier, M. S., Lebrasseur, N. K., Jasuja, R., Ruderman, N. B., \& Ido, Y. (2011). Acute exercise activates AMPK and eNOS in the mouse aorta. American Journal of Physiology. Heart and Circulatory Physiology, 301(4), H1255-65. doi:10.1152/ajpheart.01279.2010 [doi]

Cai, H., \& Harrison, D. G. (2000). Endothelial dysfunction in cardiovascular diseases: The role of oxidant stress. Circulation Research, 87(10), 840-844.

Castro, M. M., Rizzi, E., Figueiredo-Lopes, L., Fernandes, K., Bendhack, L. M., Pitol, D. L., et al. (2008). Metalloproteinase inhibition ameliorates hypertension and prevents vascular dysfunction and remodeling in renovascular hypertensive rats. Atherosclerosis, 198(2), 320-331. doi:http://dx.doi.org/10.1016/j.atherosclerosis.2007.10.011

Chantler, P. D., \& Frisbee, J. C. (2015). Arterial function in cardio-metabolic diseases: From the microcirculation to the large conduits. Progress in Cardiovascular Diseases, 57(5), 489-496. doi:10.1016/j.pcad.2014.09.005 [doi]

Chrapko, W. E., Jurasz, P., Radomski, M. W., Lara, N., Archer, S. L., \& Le Mellédo, J. (2004). Decreased platelet nitric oxide synthase activity and plasma nitric oxide metabolites in major depressive disorder. Biological Psychiatry, 56(2), 129-134. 
Cicero, A. F., Derosa, G., Manca, M., Bove, M., Borghi, C., \& Gaddi, A. V. (2007). Vascular remodeling and prothrombotic markers in subjects affected by familial combined hyperlipidemia and/or metabolic syndrome in primary prevention for cardiovascular disease. Endothelium, 14(4-5), 193-198.

Cline, M. J., \& Melmon, K. L. (1966). Plasma kinins and cortisol: A possible explanation of the anti-inflammatory action of cortisol. Science (New York, N.Y.), 153(3740), $1135-1138$.

Collier, S., Kanaley, J., Carhart, R., Frechette, V., Tobin, M., Hall, A., et al. (2008). Effect of 4 weeks of aerobic or resistance exercise training on arterial stiffness, blood flow and blood pressure in pre-and stage-1 hypertensives. Journal of Human Hypertension, 22(10), 678-686.

Csiszar, A., Wang, M., Lakatta, E. G., \& Ungvari, Z. (2008). Inflammation and endothelial dysfunction during aging: Role of NF-kappaB. Journal of Applied Physiology (Bethesda, Md.: 1985), 105(4), 1333-1341. doi:10.1152/japplphysiol.90470.2008 [doi]

Dandona, P., Aljada, A., \& Bandyopadhyay, A. (2004). Inflammation: The link between insulin resistance, obesity and diabetes. Trends in Immunology, 25(1), 4-7.

D'Aquila, P., Newton, J., \& Willner, P. (1997). Diurnal variation in the effect of chronic mild stress on sucrose intake and preference. Physiology \& Behavior, 62(2), 421-426.

d'Audiffret, A. C., Frisbee, S. J., Stapleton, P. A., Goodwill, A. G., Isingrini, E., \& Frisbee, J. C. (2010). Depressive behavior and vascular dysfunction: A link between clinical depression and vascular disease? Journal of Applied Physiology (Bethesda, Md.: 1985), 108(5), 1041-1051. doi:10.1152/japplphysiol.01440.2009 [doi]

de Andrade Moraes-Teixeira, J., Félix, A., Fernandes-Santos, C., Moura, A. S., Mandarim-de-Lacerda, C. A., \& de Carvalho, J. J. (2010). Exercise training enhances elastin, fibrillin and nitric oxide in the aorta wall of spontaneously hypertensive rats. Experimental and Molecular Pathology, 89(3), 351-357.

Demirtaş, T., Utkan, T., Karson, A., Yazır, Y., Bayramgürler, D., \& Gacar, N. (2014). The link between unpredictable chronic mild stress model for depression and vascular inflammation? Inflammation, 37(5), 1432-1438.

Dickerson, S. S., \& Kemeny, M. E. (2004). Acute stressors and cortisol responses: a theoretical integration and synthesis of laboratory research. Psychological bulletin, 130(3), 355. 
Dimeo, F., Bauer, M., Varahram, I., Proest, G., \& Halter, U. (2001). Benefits from aerobic exercise in patients with major depression: A pilot study. British Journal of Sports Medicine, 35(2), 114-117.

Dinerman, J. L., Lowenstein, C. J., \& Snyder, S. H. (1993). Molecular mechanisms of nitric oxide regulation. potential relevance to cardiovascular disease. Circulation Research, 73(2), 217-222. doi:10.1161/01.RES.73.2.217

Donley, D. A., Fournier, S. B., Reger, B. L., DeVallance, E., Bonner, D. E., Olfert, I. M., et al. (2014). Aerobic exercise training reduces arterial stiffness in metabolic syndrome. Journal of Applied Physiology (Bethesda, Md.: 1985), 116(11), 13961404. doi:10.1152/japplphysiol.00151.2014 [doi]

Einhorn, MD, FACP, FACE, Daniel. (2003). American college of endocrinology position statement on the insulin resistance syndrome*. Endocrine Practice, 9(Supplement 2), 5-21.

Emanuela, F., Grazia, M., Marco, D. R., Maria Paola, L., Giorgio, F., \& Marco, B. (2012). Inflammation as a link between obesity and metabolic syndrome. Journal of Nutrition and Metabolism, 2012

Epel, E. S., McEwen, B., Seeman, T., Matthews, K., Castellazzo, G., Brownell, K. D., et al. (2000). Stress and body shape: Stress-induced cortisol secretion is consistently greater among women with central fat. Psychosomatic Medicine, 62(5), 623-632.

Esser, N., Legrand-Poels, S., Piette, J., Scheen, A. J., \& Paquot, N. (2014). Inflammation as a link between obesity, metabolic syndrome and type 2 diabetes. Diabetes Research and Clinical Practice, 105(2), 141-150.

Everson-Rose, S. A., Meyer, P. M., Powell, L. H., Pandey, D., Torrens, J. I., Kravitz, H. M., et al. (2004). Depressive symptoms, insulin resistance, and risk of diabetes in women at midlife. Diabetes Care, 27(12), 2856-2862. doi:27/12/2856 [pii]

Ferreira, I., Henry, R. M., Twisk, J. W., van Mechelen, W., Kemper, H. C., \& Stehouwer, C. D. (2005). The metabolic syndrome, cardiopulmonary fitness, and subcutaneous trunk fat as independent determinants of arterial stiffness: The amsterdam growth and health longitudinal study. Archives of Internal Medicine, 165(8), 875-882.

Few, J. D. (1974). Effect of exercise on the secretion and metabolism of cortisol in man. The Journal of Endocrinology, 62(2), 341-353.

Fleg, J. L. (2012). Aerobic exercise in the elderly: A key to successful aging. Discovery Medicine, 13(70), 223-228.

Frisbee, J. C. (2005). Hypertension-independent microvascular rarefaction in the obese zucker rat model of the metabolic syndrome. Microcirculation, 12(5), 383-392. 
Frisbee, J. C., Samora, J. B., Peterson, J., \& Bryner, R. (2006). Exercise training blunts microvascular rarefaction in the metabolic syndrome. American Journal of Physiology.Heart and Circulatory Physiology, 291(5), H2483-92. doi:00566.2006 [pii]

Frisbee, J. C., \& Stepp, D. W. (2001). Impaired NO-dependent dilation of skeletal muscle arterioles in hypertensive diabetic obese zucker rats. American Journal of Physiology. Heart and Circulatory Physiology, 281(3), H1304-11.

Fuentes, E., Fuentes, F., Vilahur, G., Badimon, L., \& Palomo, I. (2013). Mechanisms of chronic state of inflammation as mediators that link obese adipose tissue and metabolic syndrome. Mediators of Inflammation, 2013

Galis, Z. S., \& Khatri, J. J. (2002). Matrix metalloproteinases in vascular remodeling and atherogenesis: The good, the bad, and the ugly. Circulation Research, 90(3), 251262.

Go, A. S., Mozaffarian, D., Roger, V. L., Benjamin, E. J., Berry, J. D., Blaha, M. J., et al. (2014). Executive summary: Heart disease and stroke Statistics-2014 update: A report from the american heart association. Circulation, 129(3), 399-410. doi:10.1161/01.cir.0000442015.53336.12

Golbidi, S., Frisbee, J. C., \& Laher, I. (2015). Chronic stress impacts the cardiovascular system: Animal models and clinical outcomes. American Journal of Physiology. Heart and Circulatory Physiology, 308(12), H1476-98. doi:10.1152/ajpheart.00859.2014 [doi]

Goldberg, R. B. (2009). Cytokine and cytokine-like inflammation markers, endothelial dysfunction, and imbalanced coagulation in development of diabetes and its complications. The Journal of Clinical Endocrinology \& Metabolism, 94(9), 31713182.

Goldin, A., Beckman, J. A., Schmidt, A. M., \& Creager, M. A. (2006). Advanced glycation end products: Sparking the development of diabetic vascular injury. Circulation, 114(6), 597-605. doi:114/6/597 [pii]

Gomes, V. A., Casella-Filho, A., Chagas, A. C., \& Tanus-Santos, J. E. (2008). Enhanced concentrations of relevant markers of nitric oxide formation after exercise training in patients with metabolic syndrome. Nitric Oxide, 19(4), 345-350.

Gonçalves, F. M., Jacob-Ferreira, A. L. B., Gomes, V. A., Casella-Filho, A., Chagas, A. C. P., Marcaccini, A. M., et al. (2009). Increased circulating levels of matrix metalloproteinase (MMP)-8, MMP-9, and pro-inflammatory markers in patients with metabolic syndrome. Clinica Chimica Acta, 403(1-2), 173-177. doi:http://dx.doi.org/10.1016/j.cca.2009.02.013 
Grundy, S. M., Brewer, H. B., Cleeman, J. I., Smith, S. C., \& Lenfant, C. (2004). Definition of metabolic syndrome report of the national heart, lung, and blood Institute/American heart association conference on scientific issues related to definition. Circulation, 109(3), 433-438.

Hadi, H. A., Carr, C. S., \& Al Suwaidi, J. (2005). Endothelial dysfunction: Cardiovascular risk factors, therapy, and outcome. Vascular Health and Risk Management, 1(3), 183198.

Haffner, S. M. (2006). The metabolic syndrome: Inflammation, diabetes mellitus, and cardiovascular disease. The American Journal of Cardiology, 97(2), 3-11.

Hambrecht, R., Wolf, A., Gielen, S., Linke, A., Hofer, J., Erbs, S., et al. (2000). Effect of exercise on coronary endothelial function in patients with coronary artery disease. New England Journal of Medicine, 342(7), 454-460.

Hamer, M., Batty, G. D., Seldenrijk, A., \& Kivimaki, M. (2011). Antidepressant medication use and future risk of cardiovascular disease: The scottish health survey. European Heart Journal, 32(4), 437-442. doi:10.1093/eurheartj/ehq438 [doi]

Harrington, R. (2001). Depression, suicide and deliberate self-harm in adolescence. British Medical Bulletin, 57, 47-60.

Hasenfuss, G. (1998). Animal models of human cardiovascular disease, heart failure and hypertrophy. Cardiovascular Research, 39(1), 60-76. doi:S0008-6363(98)00110-2 [pii]

Hayashi, K., Sugawara, J., Komine, H., Maeda, S., \& Yokoi, T. (2005). Effects of aerobic exercise training on the stiffness of central and peripheral arteries in middle-aged sedentary men. The Japanese Journal of Physiology, 55(4), 235-239.

Heagerty, A. M., Aalkjaer, C., Bund, S. J., Korsgaard, N., \& Mulvany, M. J. (1993). Small artery structure in hypertension. dual processes of remodeling and growth. Hypertension, 21(4), 391-397.

Heinonen, I., Rinne, P., Ruohonen, S., Ruohonen, S., Ahotupa, M., \& Savontaus, E. (2014). The effects of equal caloric high fat and western diet on metabolic syndrome, oxidative stress and vascular endothelial function in mice. Acta Physiologica, 211(3), 515-527.

Heitzer, T., Schlinzig, T., Krohn, K., Meinertz, T., \& Munzel, T. (2001). Endothelial dysfunction, oxidative stress, and risk of cardiovascular events in patients with coronary artery disease. Circulation, 104(22), 2673-2678. 
Herva, A., Laitinen, J., Miettunen, J., Veijola, J., Karvonen, J., Läksy, K., et al. (2006). Obesity and depression: Results from the longitudinal northern finland 1966 birth cohort study. International Journal of Obesity, 30(3), 520-527.

Hill, B. J., Dixon, J. L., \& Sturek, M. (2001). Effect of atorvastatin on intracellular calcium uptake in coronary smooth muscle cells from diabetic pigs fed an atherogenic diet. Atherosclerosis, 159(1), 117-124.

Hill, E., Zack, E., Battaglini, C., Viru, M., Viru, A., \& Hackney, A. (2008). Exercise and circulating cortisol levels: The intensity threshold effect. Journal of Endocrinological Investigation, 31(7), 587-591.

Isingrini, E., Surget, A., Belzung, C., Freslon, J., Frisbee, J., O'Donnell, J., et al. (2011). Altered aortic vascular reactivity in the unpredictable chronic mild stress model of depression in mice: UCMS causes relaxation impairment to ACh. Physiology \& Behavior, 103(5), 540-546.

Isomaa, B., Almgren, P., Tuomi, T., Forsén, B., Lahti, K., Nissén, M., et al. (2001). Cardiovascular morbidity and mortality associated with the metabolic syndrome. Diabetes Care, 24(4), 683-689. doi:10.2337/diacare.24.4.683

Jiang, W., Kuchibhatla, M., Cuffe, M. S., Christopher, E. J., Alexander, J. D., Clary, G. L., et al. (2004). Prognostic value of anxiety and depression in patients with chronic heart failure. Circulation, 110(22), 3452-3456. doi:01.CIR.0000148138.25157.F9 [pii]

Joannides, R., Haefeli, W. E., Linder, L., Richard, V., Bakkali, E. H., Thuillez, C., \& Lüscher, T. F. (1995). Nitric oxide is responsible for flow-dependent dilatation of human peripheral conduit arteries in vivo. Circulation, 91(5), 1314-1319.

Johnson, J. L., Slentz, C. A., Houmard, J. A., Samsa, G. P., Duscha, B. D., Aiken, L. B., et al. (2007). Exercise training amount and intensity effects on metabolic syndrome (from studies of a targeted risk reduction intervention through defined exercise). The American Journal of Cardiology, 100(12), 1759-1766.

Kangas, P., Tikkakoski, A. J., Tahvanainen, A. M., Leskinen, M. H., Viitala, J. M., Kähönen, M., et al. (2013). Metabolic syndrome may be associated with increased arterial stiffness even in the absence of hypertension: A study in 84 cases and 82 controls. Metabolism, 62(8), 1114-1122.

Katzmarzyk, P. T., Leon, A. S., Wilmore, J. H., Skinner, J. S., Rao, D., Rankinen, T., et al. (2003). Targeting the metabolic syndrome with exercise: Evidence from the HERITAGE family study. Medicine and Science in Sports and Exercise, 35(10), 17031709. 
Kauhanen, M., Korpelainen, J. T., Hiltunen, P., Brusin, E., Mononen, H., Maatta, R., et al. (1999). Poststroke depression correlates with cognitive impairment and neurological deficits. Stroke; a Journal of Cerebral Circulation, 30(9), 1875-1880.

Kaur, J. (2014). A comprehensive review on metabolic syndrome. Cardiology Research and Practice, 2014, 943162. doi:10.1155/2014/943162 [doi]

Kern, P. A., Ranganathan, S., Li, C., Wood, L., \& Ranganathan, G. (2001). Adipose tissue tumor necrosis factor and interleukin- 6 expression in human obesity and insulin resistance. American Journal of Physiology.Endocrinology and Metabolism, 280(5), E745-51.

Kessler, R. C., Berglund, P., Demler, O., Jin, R., Merikangas, K. R., \& Walters, E. E. (2005). Lifetime prevalence and age-of-onset distributions of DSM-IV disorders in the national comorbidity survey replication. Archives of General Psychiatry, 62(6), 593602.

Kessler, R. C., \& Bromet, E. J. (2013). The epidemiology of depression across cultures. Annual Review of Public Health, 34, 119-138. doi:10.1146/annurev-publhealth031912-114409 [doi]

Kinder, L. S., Carnethon, M. R., Palaniappan, L. P., King, A. C., \& Fortmann, S. P. (2004). Depression and the metabolic syndrome in young adults: Findings from the third national health and nutrition examination survey. Psychosomatic Medicine, 66(3), 316-322.

Kindermann, W., Schnabel, A., Schmitt, W., Biro, G., Cassens, J., \& Weber, F. (1982). Catecholamines, growth hormone, cortisol, insulin, and sex hormones in anaerobic and aerobic exercise. European Journal of Applied Physiology and Occupational Physiology, 49(3), 389-399.

Kochanek, K. D., Murphy, S. L., Xu, J., \& Arias, E. (2014). Mortality in the united states, 2013. NCHS Data Brief, 178, 1-8.

Koponen, H., Jokelainen, J., Keinanen-Kiukaanniemi, S., Kumpusalo, E., \& Vanhala, M. (2008). Metabolic syndrome predisposes to depressive symptoms: A populationbased 7-year follow-up study. The Journal of Clinical Psychiatry, 69(2), 178-182. doi:ej07m03309 [pii]

Körner, A., Kratzsch, J., Gausche, R., Schaab, M., Erbs, S., \& Kiess, W. (2007). New predictors of the metabolic syndrome in children-role of adipocytokines. Pediatric Research, 61(6), 640-645.

Lafont, A., Durand, E., Samuel, J. L., Besse, B., Addad, F., Levy, B. I., et al. (1999). Endothelial dysfunction and collagen accumulation: Two independent factors for 
restenosis and constrictive remodeling after experimental angioplasty. Circulation, 100(10), 1109-1115.

Lakatta, E. G. (1990). Changes in cardiovascular function with aging. European heart journal, 11(suppl C), 22-29.

Lancaster, J. R. (1997). A tutorial on the diffusibility and reactivity of free nitric oxide. Nitric Oxide, 1(1), 18-30.

Lehmann, E., Parker, J., Hopkins, K., Taylor, M., \& Gosling, R. (1993). Validation and reproducibility of pressure-corrected aortic distensibility measurements using pulsewave-velocity doppler ultrasound. Journal of Biomedical Engineering, 15(3), 221228.

Lundberg, J. O., Weitzberg, E., \& Gladwin, M. T. (2008). The nitrate-nitrite-nitric oxide pathway in physiology and therapeutics. Nature Reviews Drug Discovery, 7(2), 156167.

Madden, K. M., Lockhart, C., Cuff, D., Potter, T. F., \& Meneilly, G. S. (2009). Short-term aerobic exercise reduces arterial stiffness in older adults with type 2 diabetes, hypertension, and hypercholesterolemia. Diabetes Care, 32(8), 1531-1535. doi:10.2337/dc09-0149 [doi]

Majumder, K., \& Wu, J. (2014). Molecular targets of antihypertensive peptides: Understanding the mechanisms of action based on the pathophysiology of hypertension. International Journal of Molecular Sciences, 16(1), 256-283.

Malik, S., Wong, N. D., Franklin, S. S., Kamath, T. V., L'Italien, G. J., Pio, J. R., et al. (2004). Impact of the metabolic syndrome on mortality from coronary heart disease, cardiovascular disease, and all causes in united states adults. Circulation, 110(10), 1245-1250. doi:10.1161/01.CIR.0000140677.20606.0E [doi]

Miller, A. H., Maletic, V., \& Raison, C. L. (2009). Inflammation and its discontents: The role of cytokines in the pathophysiology of major depression. Biological Psychiatry, 65(9), 732-741.

Mozaffarian, D., Benjamin, E. J., Go, A. S., Arnett, D. K., Blaha, M. J., Cushman, M., et al. (2015). Heart disease and stroke statistics--2015 update: A report from the american heart association. Circulation, 131(4), e29-322. doi:10.1161/CIR.0000000000000152 [doi]

Osol, G., \& Mandala, M. (2009). Maternal uterine vascular remodeling during pregnancy. Physiology, 24(1), 58-71.

Palmer, R. M., Ashton, D., \& Moncada, S. (1988). Vascular endothelial cells synthesize nitric oxide from L-arginine. Nature, 333(6174), 664-666. 
Paredes, S., \& Ribeiro, L. (2014). Cortisol: The villain in metabolic syndrome? Revista Da Associação Médica Brasileira, 60(1), 84-92.

Peeling, P., Dawson, B., Goodman, C., Landers, G., Wiegerinck, E. T., Swinkels, D. W., et al. (2009). Training surface and intensity: Inflammation, hemolysis, and hepcidin expression. Medicine and Science in Sports and Exercise, 41(5), 1138-1145. doi:10.1249/MSS.0b013e318192ce58 [doi]

Phillips, S. A., Sylvester, F. A., \& Frisbee, J. C. (2005). Oxidant stress and constrictor reactivity impair cerebral artery dilation in obese zucker rats. American Journal of Physiology.Regulatory, Integrative and Comparative Physiology, 288(2), R522-30. doi:00655.2004 [pii]

Picchi, A., Gao, X., Belmadani, S., Potter, B. J., Focardi, M., Chilian, W. M., et al. (2006). Tumor necrosis factor-alpha induces endothelial dysfunction in the prediabetic metabolic syndrome. Circulation Research, 99(1), 69-77. doi:01.RES.0000229685.37402.80 [pii]

Pradhan, A. D., Manson, J. E., Rifai, N., Buring, J. E., \& Ridker, P. M. (2001). C-reactive protein, interleukin 6, and risk of developing type 2 diabetes mellitus. Jama, 286(3), 327-334.

Prakhinkit, S., Suppapitiporn, S., Tanaka, H., \& Suksom, D. (2014). Effects of buddhism walking meditation on depression, functional fitness, and endothelium-dependent vasodilation in depressed elderly. The Journal of Alternative and Complementary Medicine, 20(5), 411-416.

Prasad, A., \& Quyyumi, A. A. (2004). Renin-angiotensin system and angiotensin receptor blockers in the metabolic syndrome. Circulation, 110(11), 1507-1512. doi:10.1161/01.CIR.0000141736.76561.78 [doi]

Prior, B. M., Lloyd, P. G., Yang, H., \& Terjung, R. L. (2003). Exercise-induced vascular remodeling. Exercise and Sport Sciences Reviews, 31(1), 26-33.

Raikkonen, K., Matthews, K. A., \& Kuller, L. H. (2007). Depressive symptoms and stressful life events predict metabolic syndrome among middle-aged women: A comparison of world health organization, adult treatment panel III, and international diabetes foundation definitions. Diabetes Care, 30(4), 872-877. doi:30/4/872 [pii]

Rajagopalan, S., Brook, R., Rubenfire, M., Pitt, E., Young, E., \& Pitt, B. (2001). Abnormal brachial artery flow-mediated vasodilation in young adults with major depression. The American Journal of Cardiology, 88(2), 196-198.

Rajagopalan, S., Meng, X. P., Ramasamy, S., Harrison, D. G., \& Galis, Z. S. (1996). Reactive oxygen species produced by macrophage-derived foam cells regulate the activity of vascular matrix metalloproteinases in vitro. implications for atherosclerotic 
plaque stability. The Journal of Clinical Investigation, 98(11), 2572-2579. doi:10.1172/JCl119076 [doi]

Reddy, G. K. (2004). AGE-related cross-linking of collagen is associated with aortic wall matrix stiffness in the pathogenesis of drug-induced diabetes in rats. Microvascular Research, 68(2), 132-142.

Rees, D. D., Palmer, R. M., \& Moncada, S. (1989). Role of endothelium-derived nitric oxide in the regulation of blood pressure. Proceedings of the National Academy of Sciences of the United States of America, 86(9), 3375-3378.

Roberts, C. K., Won, D., Pruthi, S., Kurtovic, S., Sindhu, R. K., Vaziri, N. D., et al. (2006). Effect of a short-term diet and exercise intervention on oxidative stress, inflammation, MMP-9, and monocyte chemotactic activity in men with metabolic syndrome factors. Journal of Applied Physiology (Bethesda, Md.: 1985), 100(5), 1657-1665. doi:01292.2005 [pii]

Rodríguez, D., Morrison, C. J., \& Overall, C. M. (2010). Matrix metalloproteinases: What do they not do? new substrates and biological roles identified by murine models and proteomics. Biochimica Et Biophysica Acta (BBA) - Molecular Cell Research, 1803(1), 39-54. doi:http://dx.doi.org/10.1016/j.bbamcr.2009.09.015

Rudic, R. D., Shesely, E. G., Maeda, N., Smithies, O., Segal, S. S., \& Sessa, W. C. (1998). Direct evidence for the importance of endothelium-derived nitric oxide in vascular remodeling. The Journal of Clinical Investigation, 101(4), 731-736. doi:10.1172/JCl1699 [doi]

Russell, J. C., \& Proctor, S. D. (2006). Small animal models of cardiovascular disease: Tools for the study of the roles of metabolic syndrome, dyslipidemia, and atherosclerosis. Cardiovascular Pathology, 15(6), 318-330.

Rybakowski, J. K., Wykretowicz, A., Heymann-Szlachcinska, A., \& Wysocki, H. (2006). Impairment of endothelial function in unipolar and bipolar depression. Biological Psychiatry, 60(8), 889-891.

Sachar, E. J., Hellman, L., Roffwarg, H. P., Halpern, F. S., Fukushima, D. K., \& Gallagher, T. F. (1973). Disrupted 24-hour patterns of cortisol secretion in psychotic depression. Archives of General Psychiatry, 28(1), 19-24.

Schillaci, G., Pirro, M., Vaudo, G., Mannarino, M. R., Savarese, G., Pucci, G., et al. (2005). Metabolic syndrome is associated with aortic stiffness in untreated essential hypertension. Hypertension, 45(6), 1078-1082. doi:01.HYP.0000165313.84007.7d [pii] 
Seldenrijk, A., van Hout, H. P., van Marwijk, H. W., de Groot, E., Gort, J., Rustemeijer, C., et al. (2011). Depression, anxiety, and arterial stiffness. Biological Psychiatry, 69(8), 795-803.

Sessa, W. C., Pritchard, K., Seyedi, N., Wang, J., \& Hintze, T. H. (1994). Chronic exercise in dogs increases coronary vascular nitric oxide production and endothelial cell nitric oxide synthase gene expression. Circulation Research, 74(2), 349-353.

Sherwood, A., Hinderliter, A. L., Watkins, L. L., Waugh, R. A., \& Blumenthal, J. A. (2005). Impaired endothelial function in coronary heart disease patients with depressive symptomatology. Journal of the American College of Cardiology, 46(4), 656-659.

Shimbo, D., Chaplin, W., Crossman, D., Haas, D., \& Davidson, K. W. (2005). Role of depression and inflammation in incident coronary heart disease events. The American Journal of Cardiology, 96(7), 1016-1021.

Skilton, M. R., Moulin, P., Terra, J., \& Bonnet, F. (2007). Associations between anxiety, depression, and the metabolic syndrome. Biological Psychiatry, 62(11), 1251-1257.

Stehouwer, C., Henry, R., \& Ferreira, I. (2008). Arterial stiffness in diabetes and the metabolic syndrome: A pathway to cardiovascular disease. Diabetologia, 51(4), 527539.

Steinberg, H. O., Brechtel, G., Johnson, A., Fineberg, N., \& Baron, A. D. (1994). Insulinmediated skeletal muscle vasodilation is nitric oxide dependent. A novel action of insulin to increase nitric oxide release. The Journal of Clinical Investigation, 94(3), 1172-1179. doi:10.1172/JCl117433 [doi]

Stewart, J. C., Rand, K. L., Muldoon, M. F., \& Kamarck, T. W. (2009). A prospective evaluation of the directionality of the depression-inflammation relationship. Brain, Behavior, and Immunity, 23(7), 936-944.

Stokes, P. E. (1995). The potential role of excessive cortisol induced by HPA hyperfunction in the pathogenesis of depression. European Neuropsychopharmacology, 5, 77-82.

Ströhle, A. (2009). Physical activity, exercise, depression and anxiety disorders. Journal of Neural Transmission, 116(6), 777-784.

Stühlinger, M. C., Abbasi, F., Chu, J. W., Lamendola, C., McLaughlin, T. L., Cooke, J. P., et al. (2002). Relationship between insulin resistance and an endogenous nitric oxide synthase inhibitor. Jama, 287(11), 1420-1426.

Stühlinger, M. C., Abbasi, F., Chu, J. W., Lamendola, C., McLaughlin, T. L., Cooke, J. P., et al. (2002). Relationship between insulin resistance and an endogenous nitric oxide synthase inhibitor. Jama, 287(11), 1420-1426. 
Tayebjee, M. H., Lip, G. Y., Blann, A. D., \& MacFadyen, R. J. (2005). Effects of age, gender, ethnicity, diurnal variation and exercise on circulating levels of matrix metalloproteinases (MMP)-2 and-9, and their inhibitors, tissue inhibitors of matrix metalloproteinases (TIMP)-1 and-2. Thrombosis Research, 115(3), 205-210.

Tiemeier, H., Breteler, M., Van Popele, N. M., Hofman, A., \& Witteman, J. (2003). LateLife depression is associated with arterial stiffness: A Population-Based study. Journal of the American Geriatrics Society, 51(8), 1105-1110.

Tjonna, A. E., Lee, S. J., Rognmo, O., Stolen, T. O., Bye, A., Haram, P. M., et al. (2008). Aerobic interval training versus continuous moderate exercise as a treatment for the metabolic syndrome: A pilot study. Circulation, 118(4), 346-354. doi:10.1161/CIRCULATIONAHA.108.772822 [doi]

Touati, S., Meziri, F., Devaux, S., Berthelot, A., Touyz, R. M., \& Laurant, P. (2011). Exercise reverses metabolic syndrome in high-fat diet-induced obese rats. Medicine and Science in Sports and Exercise, 43(3), 398-407. doi:10.1249/MSS.0b013e3181eeb12d [doi]

Tounian, P., Aggoun, Y., Dubern, B., Varille, V., Guy-Grand, B., Sidi, D., et al. (2001). Presence of increased stiffness of the common carotid artery and endothelial dysfunction in severely obese children: A prospective study. The Lancet, 358(9291), 1400-1404.

Tronc, F., Wassef, M., Esposito, B., Henrion, D., Glagov, S., \& Tedgui, A. (1996). Role of NO in flow-induced remodeling of the rabbit common carotid artery. Arteriosclerosis, Thrombosis, and Vascular Biology, 16(10), 1256-1262.

Troseid, M., Seljeflot, I., Hjerkinn, E. M., \& Arnesen, H. (2009). Interleukin-18 is a strong predictor of cardiovascular events in elderly men with the metabolic syndrome: Synergistic effect of inflammation and hyperglycemia. Diabetes Care, 32(3), 486-492. doi:10.2337/dc08-1710 [doi]

Tuttolomondo, A., Di Raimondo, D., Di Sciacca, R., Pecoraro, R., Arnao, V., Buttà, C., et al. (2012). Arterial stiffness and ischemic stroke in subjects with and without metabolic syndrome. Atherosclerosis, 225(1), 216-219.

Vaccarino, V., Johnson, B. D., Sheps, D. S., Reis, S. E., Kelsey, S. F., Bittner, V., et al. (2007). Depression, inflammation, and incident cardiovascular disease in women with suspected coronary ischemia: The national heart, lung, and blood Institutesponsored WISE study. Journal of the American College of Cardiology, 50(21), 20442050.

van Varik, B. J., Rennenberg, R. J., Reutelingsperger, C. P., Kroon, A. A., de Leeuw, P. W., \& Schurgers, L. J. (2012). Mechanisms of arterial remodeling: Lessons from genetic diseases. Frontiers in Genetics, 3, 290. doi:10.3389/fgene.2012.00290 [doi] 
Vozarova, B., Weyer, C., Hanson, K., Tataranni, P. A., Bogardus, C., \& Pratley, R. E. (2001). Circulating interleukin-6 in relation to adiposity, insulin action, and insulin secretion. Obesity Research, 9(7), 414-417.

Waloszek, J. M., Byrne, M. L., Woods, M. J., Nicholas, C. L., Bei, B., Murray, G., et al. (2015). Early physiological markers of cardiovascular risk in community based adolescents with a depressive disorder. Journal of Affective Disorders, 175, 403-410.

Wang, P. S., Simon, G., \& Kessler, R. C. (2003). The economic burden of depression and the cost-effectiveness of treatment. International Journal of Methods in Psychiatric Research, 12(1), 22-33. doi:10.1002/mpr.139

Weiss, T. W., Arnesen, H., \& Seljeflot, I. (2013). Components of the interleukin-6 transsignalling system are associated with the metabolic syndrome, endothelial dysfunction and arterial stiffness. Metabolism, 62(7), 1008-1013.

Weiss, T. W., Arnesen, H., Trøseid, M., Kaun, C., Hjerkinn, E. M., Huber, K., et al. (2011). Adipose tissue expression of interleukin-18 mRNA is elevated in subjects with metabolic syndrome and independently associated with fasting glucose. Wiener Klinische Wochenschrift, 123(21-22), 650-654.

Weitzman, E. D., Fukushima, D., Nogeire, C., Roffwarg, H., Gallagher, T., \& Hellman, L. (1971). Twenty-four hour pattern of the episodic secretion of cortisol in normal subjects. The Journal of Clinical Endocrinology \& Metabolism, 33(1), 14-22.

Whitehead, J., Richards, A., Hickman, I., Macdonald, G., \& Prins, J. (2006). Adiponectina key adipokine in the metabolic syndrome. Diabetes, Obesity and Metabolism, 8(3), 264-280.

Williams, S. B., Cusco, J. A., Roddy, M., Johnstone, M. T., \& Creager, M. A. (1996). Impaired nitric oxide-mediated vasodilation in patients with non-insulin-dependent diabetes mellitus. Journal of the American College of Cardiology, 27(3), 567-574.

Willner, P. (1997). Validity, reliability and utility of the chronic mild stress model of depression: A 10-year review and evaluation. Psychopharmacology, 134(4), 319329.

Willner, P., Muscat, R., \& Papp, M. (1992). Chronic mild stress-induced anhedonia: A realistic animal model of depression. Neuroscience \& Biobehavioral Reviews, 16(4), 525-534.

Yaffe, K., Kanaya, A., Lindquist, K., Simonsick, E. M., Harris, T., Shorr, R. I., et al. (2004). The metabolic syndrome, inflammation, and risk of cognitive decline. Jama, 292(18), 2237-2242. 
Yang, H. T., Yan, Z., Abraham, J. A., \& Terjung, R. L. (2001). VEGF(121)- and bFGFinduced increase in collateral blood flow requires normal nitric oxide production. American Journal of Physiology. Heart and Circulatory Physiology, 280(3), H1097104.

Yehuda, R., Teicher, M. H., Trestman, R. L., Levengood, R. A., \& Siever, L. J. (1996). Cortisol regulation in posttraumatic stress disorder and major depression: $\mathrm{A}$ chronobiological analysis. Biological Psychiatry, 40(2), 79-88.

Yeragani, V. K., Tancer, M., Seema, K., Josyulab, K., \& Desai, N. (2006). Increased pulse-wave velocity in patients with anxiety: Implications for autonomic dysfunction. Journal of Psychosomatic Research, 61(1), 25-31.

Zaragoza, C., Gomez-Guerrero, C., Martin-Ventura, J. L., Blanco-Colio, L., Lavin, B., Mallavia, B., et al. (2011). Animal models of cardiovascular diseases. Journal of Biomedicine \& Biotechnology, 2011, 497841. doi:10.1155/2011/497841 [doi]

Zieman, S. J., \& Kass, D. A. (2004). Advanced glycation endproduct crosslinking in the cardiovascular system. Drugs, 64(5), 459-470.

Zieman, S. J., Melenovsky, V., \& Kass, D. A. (2005). Mechanisms, pathophysiology, and therapy of arterial stiffness. Arteriosclerosis, Thrombosis, and Vascular Biology, 25(5), 932-943. doi:10.1161/01.ATV.0000160548.78317.29

Zoppini, G., Targher, G., Zamboni, C., Venturi, C., Cacciatori, V., Moghetti, P., et al. (2006). Effects of moderate-intensity exercise training on plasma biomarkers of inflammation and endothelial dysfunction in older patients with type 2 diabetes. Nutrition, Metabolism and Cardiovascular Diseases, 16(8), 543-549. 
Page left intentionally blank 


\section{Chapter 3:}

\section{Aerobic Exercise Intervention Improves}

Structural Carotid Artery Remodeling in a Rodent Model of Metabolic Syndrome

\section{and Chronic Stress}




\begin{abstract}
Metabolic Syndrome (MetS) and chronic stress/depression are associated with an increased risk for poor cardiovascular outcomes and harmful arterial remodeling. Both of these diseases are continuously increasing in prevalence, but not much is known about the pathological effects of their comorbidity. Exercise is a widely accepted and supported modality for combating MetS and managing chronic stress. However, a gap in the literature exists regarding the degree to which exercise can mitigate the injurious arterial remodeling associated with MetS and chronic stress simultaneously. The Unpredictable Chronic Mild Stress (UCMS) model is used as a simulation of daily, prolonged stress. The Obese Zucker Rat (OZR) represents MetS, with Lean Zucker Rats (LZR) paralleling a healthy individual. PURPOSE: To determine the degree to which exercise can attenuate negative arterial remodeling caused by chronic stress and metabolic syndrome. METHODS: OZRs and LZRs were separated into experimental groups including: control (C), exercise (Ex), UCMS, and UCMS with exercise (UCMS+Ex). The carotid artery was isolated and passive vessel mechanic responses to changes in intraluminal pressure were assessed. RESULTS: OZR-UCMS inner diameter (ID) was significantly smaller when compared to LZR-C, Ex, and UCMS+Ex. OZR-UCMS wall thickness (WT) and wall:Iumen ratio (WLR) was significantly higher than all LZR groups, but was rescued with Ex training, regardless of UCMS. CONCLUSIONS: Comorbidity between MetS and chronic stress is a confounding factor on healthy vasculature, and can cause hypertrophic remodeling of the carotid artery. Exercise can be used as an intervention for these two diseases to mitigate adverse carotid artery remodeling.
\end{abstract}


Introduction

Metabolic Syndrome (MetS) and depression are both risk factors for cardiovascular disease (CVD), and have been bi-directionally linked to each other (Dunbar, et al., 2008). The increased risk of CVD in MetS (Stehouwer, et al., 2008) and depression (Seldenrijk, et al., 2011) has been linked to increases in arterial stiffness and adverse changes to vascular structure (Scuteri, et al., 2004), resulting in further complications, including myocardial infarction and stroke.

The vascular dysfunction associated with MetS and depression has been shown to be prevalent in the conduit arteries (Schillaci, et al., 2005 \& Stanley, et al., 2014). The conduit, or elastic arteries, are responsible for maintaining a constant pressure throughout the arterial system, and include the carotid arteries and aorta. Alterations to conduit function can result in pathological conditions and alterations in normal cardiac function and blood flow. MetS is associated with an increase in carotid intima media thickness (IMT) and advanced vascular damage (Oliijhoek, et al., 2004).

Moderate intensity aerobic exercise has been shown to have beneficial effects on the vasculature of individuals with MetS (Donley, et al., 2014) and depressive-like symptoms (Kiuchi, et al., 2012). Furthermore, exercise has been shown to be neuroprotective (Teri, et al., 2003; Nabkasorn, et al., 2006) and to have a favorable effect on the majority of risk factors associated with MetS (Pattyn, et al., 2013).

To simulate MetS the obese Zucker rat (OZR) model was used. The OZR (fa/fa) exhibits rapid weight gain, hypertension, elevated blood glucose, and dyslipidemia making it a suitable model for MetS (Zucker, 1972). In order to simulate depression-like symptoms the unpredictable chronic mild stress (UCMS) protocol was implemented. The UCMS protocol produces depressive-like symptoms in rodents through decreases in 
award seeking, increased HPA axis activity, adrenal hypertrophy, and increases in corticosterone production (Willner, 1997).

The goal of this study was to fully assess the structural and molecular changes in the carotid artery caused by depression and MetS as comorbidities, and evaluate the effectiveness of moderate intensity aerobic exercise on the vasculature of this comorbid state. To simulate MetS the OZR, which displays elevated blood glucose, hypertension, rapid weight gain, dyslipidemia, and increased inflammation, was used, with the LZR serving as the healthy control. Furthermore, the UCMS protocol was utilized to induce depression-like symptoms in the animals. We hypothesized the comorbid state of MetS and depression would result in increased adverse arterial remodeling and exercise training would be able to attenuate the negative vascular changes caused by the comorbidities.

Materials and Methods

Animals and Housing: Male LZR and OZR (Lepr ${ }^{\text {fafaa }}$ ) were purchased from Envigo Labratories (Indianapolis, IN) and housed in a pathogen-free vivarium room with a 12hr:12hr reverse light:dark cycle, in standard Allentown rat cages.

Feeding and handling: Rats were fed standard rodent chow and tap water ad libitum. Animal handling and care were performed in accordance with the Public Health Service Policy on Humane Care and Use of Laboratory Animals and West Virginia University's Institution of Animal Care and Use Committee. 
Study Design: Rodents were divided into eight experimental groups: LZR control (C) $(n=8)$, LZR exercise (EX) (n=8), LZR UCMS ( $n=8)$, LZR EX+UCMS ( $n=8)$, OZR C $(n=8)$, OZR EX $(n=8)$, OZR UCMS $(n=8)$, and OZR UCMS+EX $(n=10)$. Animals were single housed. Rats were weighed and their weights recorded weekly for the duration of the study. To ensure animals were being adequately fed food amount was weighed and recorded twice a week. Control animals remained in the vivarium room and were only disturbed for body and food weighing, and coat scoring throughout the duration of the study.

Coat Scoring: Coat score data was obtained weekly to determine the health and grooming habits of each animals. The total cumulative score was determined by giving an individual score of "0" (clean) or "1" (dirty) to eight different body areas (head, neck, back, underside, tail, hindlimb, forelimb, and genitals).

Veterinary Treatment: If animals sustained injuries or lesions during the course of the study they were reported to WVU's Office of Laboratory Animal Research veterinary staff. The veterinary staff inspected and treated the animal. If further attention was needed directions were given and strictly adhered to.

Exercise Training: Animals were run on a Columbus Instruments rodent treadmill (Exer-3/6) for 60 minutes per day, five days per week, over the course of the eight week study. Animals were run on a slight incline of 10 degrees to place more emphasis on training the fore and hindlimb muscles. On the first day of training animals were placed 
on the treadmill for 10 minutes, running at $5 \mathrm{~m} / \mathrm{min}$. During the first week, each day time and speed were increased, until at the start of week two animals were exercising for 60 minutes, reaching a speed of $20 \mathrm{~m} / \mathrm{min}$ (LZR) or $14 \mathrm{~m} / \mathrm{min}(\mathrm{OZR})$. Animals exercised on a progressive exercise regimen, beginning each session with a 5 minute warm-up at 5 $\mathrm{m} / \mathrm{min}(\mathrm{OZR}$ ) or $7 \mathrm{~m} / \mathrm{min}$ (LZR) and progressively increasing speed throughout the session. If an animal refused to run, by sitting on the shock bay, resisting gentle prodding, the shock bay was turned off and a note was made of the animal's refusal to run.

Maximal Speed Test: To determine if a training effect was present a maximal speed test was performed during weeks one and eight. Animals began running at $5 \mathrm{~m} / \mathrm{min}$ with speed increasing by $2 \mathrm{~m} / \mathrm{min}$ every 30 seconds until exhaustion. Exhaustion was defined as when the rat sat on the shock bay with no attempts to continue exercising for 5 seconds. Maximal running speeds were recorded following exhaustion.

Work Calculation: Work $(\mathrm{J})$ was calculated in an attempt to normalize speed and body weight across all experimental groups. Work was calculated using the equation $(B M * 9.81) *\left(\sin \frac{\theta * \pi}{180}\right) * D$, where BM is body mass $(\mathrm{kg}), 9.81$ is gravity, $\theta$ is the angle of the treadmill, and $D$ is the distance run (meters, $m$ ).

Unpredictable Chronic Mild Stress (UCMS) Protocol: The UCMS protocol was used to simulate chronic stress/depression. Rodents were subjected to a variety of stresses over a 6-8 hour period five days per week. Stresses included: the removal of bedding (no bedding), the addition of $10 \mathrm{oz}$. of water to the bedding (damp bedding), the 
removal of bedding and the addition of $10 \mathrm{oz}$. of water to the cage (bath), the removal of bedding and a 45 degree cage tilt (cage tilt), relocation of rodents into another rodents cage (social stress), and successive light/dark cycles (light/dark cycle). Each stress was performed for 2-4 hours per day so animals were exposed to 2-3 stresses per day. The stresses were designed to be unpredictable to prevent acclimation to the protocol, therefore stresses were purposefully different each day with the protocol never repeating on consecutive days.

Injection, Tracheotomy, Mean Arterial Pressure, and Blood Draw: Animals were injected with sodium pentobarbital (Nembutal $50 \mathrm{mg} / \mathrm{kg}$ ) via intraperitoneal injection. Animals were inspected for consciousness via toe pinch. Booster injections were given until the rodent had no response to a substantial squeezing on the right foot with forceps. At the point of unconsciousness the animal's neck was cleaned of fur, skin, fat, muscle, and fascia until the trachea was exposed. A small incision was made in anterior wall of the trachea and a breathing tube was inserted and sutured in place. The right carotid artery was then exposed and cannulated to determine mean arterial pressure (MAP). Following a 5 minute equilibration period the MAP was recorded. Immediately following the recording of MAP an incision was made into the abdomen on the animal and the diaphragm was cut. A blood draw was then made from the inferior vena cava, which was immediately centrifuged and taken for lab analysis.

Carotid Artery Removal, Hanging, and Passive Mechanics: Following blood draw the left carotid artery was isolated in the same fashion as previously mentioned. Once 
isolated an incision on the proximal and distal end of the carotid was made and the vessel removed and placed in a dish filled with physiological salt solution (PSS). From the dish the vessel was transferred to $37^{\circ} \mathrm{C}$ microvessel chamber and superperfused with Van Breeman's calcium-free solution. The carotid was cannulated with inflow and outflow micropipettes and secured with suture. Vessels were pressurized at 100\% MAP via hydrostatic column. Passive vessel mechanic responses to changes in intraluminal pressure, of the inner (ID) and outer diameter (OD), were measured by video micrometer and recorded.

Wall Thickness: Wall thickness (WT) was calculated by the equation $\left(\frac{O D-I D}{2}\right)$, where the inner diameter was subtracted from the outer diameter, and the difference was divided by two.

Cross-Sectional Wall Area: Cross-sectional wall area (CSWA) was calculated using the equation $\left(\pi * \frac{O D}{2}\right)^{2}-\left(\pi * \frac{I D}{2}\right)^{2}$.

Wall:Lumen Ratio: Wall:Lumen Ratio (WLR) was calculated using the equation $\frac{W T}{\frac{I D}{2}}$, where the WT calculated previously was divided by the corresponding ID divided by two.

Stress-Strain Curves: For the calculation of circumferential stress intraluminal pressure was converted from $\mathrm{mmHg}$ to $\mathrm{N} / \mathrm{m}^{2}$, where $1 \mathrm{mmHg}=1.334 \times 102 \mathrm{~N} / \mathrm{m}^{2}$. 
Circumferential stress was then calculated using the equation $\sigma=\frac{\text { Pil xID }}{2 W T}$ and circumferential ID5 strain was calculated as $\varepsilon=\frac{(\mathrm{ID}-\mathrm{ID} 5)}{\mathrm{ID} 5}$, where $\mathrm{P}_{\mathrm{il}}$ represents the intraluminal pressure and ID5 represents the internal arterial diameter at the lowest intraluminal pressure (i.e. $5 \mathrm{mmHg}$ ). Each stress-strain relationship was fitted with the equation $Y=\alpha \cdot e^{\beta X}$, where $Y$ is circumferential stress, $X$ is circumferential strain, $\alpha$ is the intercept, and $\beta$ is the slop of the exponential fit. The $\beta$-coefficient is used as a relative measure of vascular stiffness.

Corticosterone ELISA: The concentration of corticosterone in the plasma was measured with an ELISA kit (Cayman Scientific). The change in color was monitored at a wavelength of $405 \mathrm{~nm}$ using a Dynex MRX plate reader. Measurements were performed in duplicate with all comparisons performed with the same assay. The corticosterone content was expressed as $\mathrm{pg} / \mathrm{ml}$ and reported as relative to LZR control values.

Statistical Analyses: All values are expressed as mean \pm SEM unless otherwise specified. A one-way ANOVA was used to compare groups unless otherwise specified. When a main effect was observed a Tukey post-hoc test was performed to determine group differences. All statistics were performed using SPSS.

Results

Body Weight: Body weight increased for all animals over the duration of study. LZR body weight remained consistent, with animals reaching roughly 400 grams, regardless of 
treatment. OZR gained weight more rapidly, with controls reaching over $600 \mathrm{~g}$ on average. The OZR UCMS group displayed a similar weight gain, averaging nearly $585 \mathrm{~g}$ by the end of the eight weeks. OZR Exercise and UCMS+Ex animals displayed a marked increase in body weight compared to OZR C, averaging nearly $630 \mathrm{~g}$ and $550 \mathrm{~g}$, respectively (Table 1).

Food Intake: OZR C was found to consume significantly more food than LZR C $(p<0.01)$. LZR UCMS consumed less food than OZR UCMS $(p<0.01)$. LZR Ex animals consumed less food than OZR Ex $(p<0.01)$. OZR Ex animals consumed less than OZR UCMS animals $(p<0.01)$. LZR UCMS+Ex consumed less than OZR UCMS+Ex (Table 1).

Work: LZR UCMS+Ex animals performed the least amount of work $(p<0.01)$. LZR Ex and OZR Ex performed the same amount of work. OZR UCMS+Ex performed significantly more work than all groups $(\mathrm{p}<0.01)($ Figure 1$)$.

Mean Arterial Pressure: OZR C had increased MAP compared to LZR C. No statistical differences were found between UCMS groups or Ex groups. OZR UCMS+Ex animals were found to have decreased MAP compared to OZR C (Table 1).

Coat Scores: LZR C animals had markedly better coat scores than all OZR groups, as well as LZR UCMS and UCMS+Ex ( $p<0.002)$. Additionally, LZR Ex animals had improved coat scores compared to the LZR groups exposed to UCMS and all OZR groups $(p<0.04)$. 
OZR C coat scores were significantly better than LZR UCMS+Ex and all other OZR Groups $(p<0.04)$ (Figure 2).

Corticosterone ELISA: LZR C animals showed markedly decreased corticosterone concentration compared to all other groups, with LZR Ex having a decrease in corticosterone when compared to LZR UCMS and UCMS+Ex $(p<0.05)$. OZR C had increased corticosterone compared to other OZR groups $(p<0.05)$, with OZR UCMS+Ex showing a decrease in corticosterone compared to OZR Ex and OZR UCMS (Figure 3).

Inner Diameter: LZR C, LZR Ex, and LZR UCMS+Ex groups were found to have a significantly larger inner diameter when compared to OZR UCMS rats $(p<0.05)$. Although not statistically significant the LZR UCMS group appeared to have a trend towards a smaller inner diameter. Additionally, OZR UCMS+Ex appeared to have a trend towards increase the inner diameter of the vessel wall (Figure 4).

Outer Diameter: No statistical differences were observed between animals, regardless of strain or treatment type (Figure 4).

Wall Thickness: OZR UCMS animals were found to have a significantly thicker wall than all other groups $(p<0.04)$, with the exception of OZR C. Exercise training was able to ameliorate the thickening of the wall caused by the combination of MetS and stress. LZR animals did not display a thickening of the arterial walls in any treatment (Figure 5). 
Wall:Lumen Ratio: The OZR UCMS group was shown to have a significantly higher WLR than all other groups, with the exception of OZR C $(p<0.009)$. Exercise training was able to improve WLR in the combination group of MetS and stress. LZR animals were resistant to changes in WLR across all treatments (Figure 6).

Cross-Sectional Wall Area: No statistical differences were observed across strain or treatment group. OZR UCMS animals appeared to have a trend towards a higher CSWA (Figure 7).

Stress:Strain Curves: No statistical differences were observed when comparing the $\beta$ slopes of stress:strain relationships in LZR animals (Figure 7). A trend did appear to show exercise training to improve the stress-strain relationship, while UCMS worsened it. However, in OZR animals Ex, UCMS, and Ex+UCMS all showed a significant improvement in $\beta$-slope values (Figure 8). 
Tables and Figures

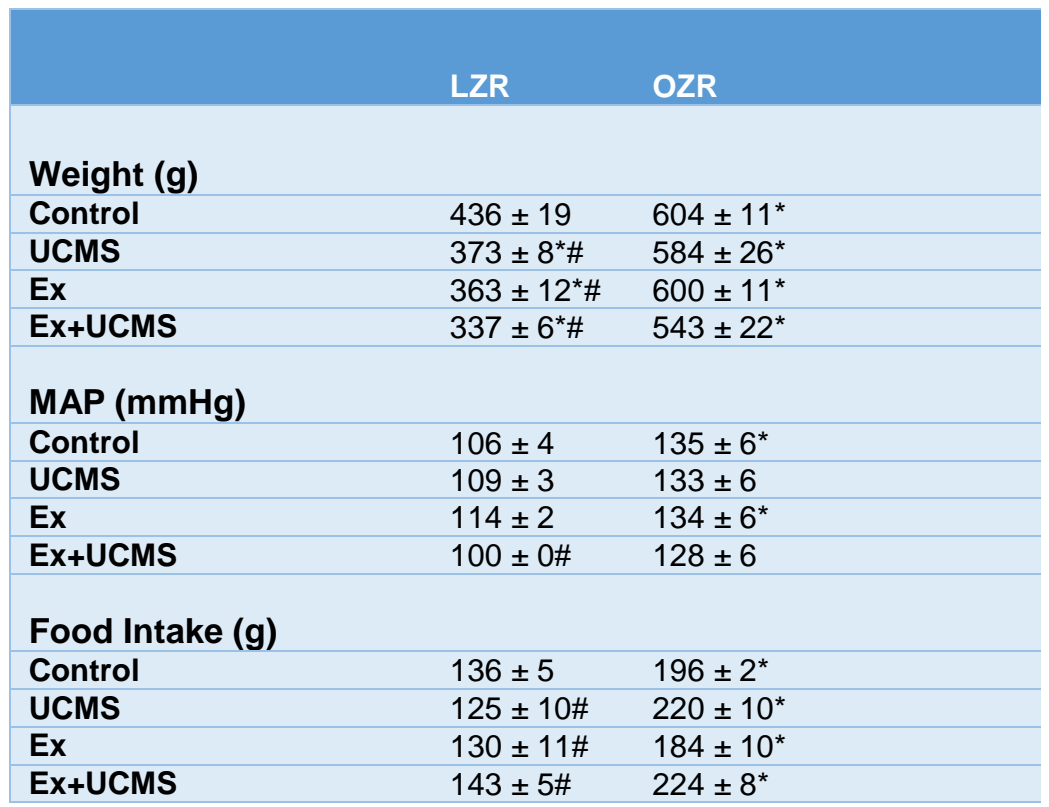

Table 1. Average body weight, mean arterial pressure (MAP), and food intake for all experimental groups. Lean Zucker rat (LZR) control (C) weighed less than all obese Zucker rat (OZR) groups and all other groups. All LZR groups weighed less than OZR C. All LZR groups had significantly lower mean arterial pressure (MAP) than OZR C. MAP did not significantly decrease from OZR $C$ in any OZR group. OZR C consumed more food than all LZR groups, but did not differ significantly from other OZR groups. ${ }^{*}=p$ $<0.05$ vs. LZR C. $\#=p<0.05$ vs. OZR C. $n=8$ for all groups.

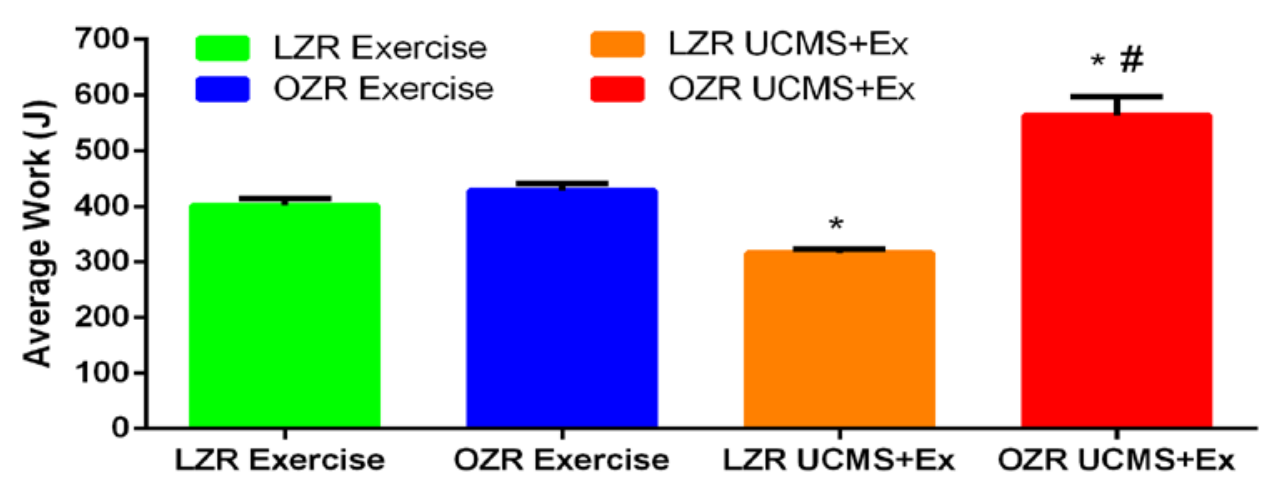

Figure 1. Average work (Joules) for each exercise treatment group. Lean Zucker rat (LZR) and obese Zucker rat (OZR) exercise performed the same amount of work during their exercise training. LZR unpredictable chronic mild stress and exercise (UCMS+Ex) performed the least amount of work compared to all groups. OZR UCMS+Ex had the highest average work. Values: mean \pm SEM. ${ }^{*}=p<0.01$ vs. LZR control; $=p<0.01$ vs. OZR control; $\#=p<0.01$ vs. LZR/OZR in treatment group. All groups $n=8$. 


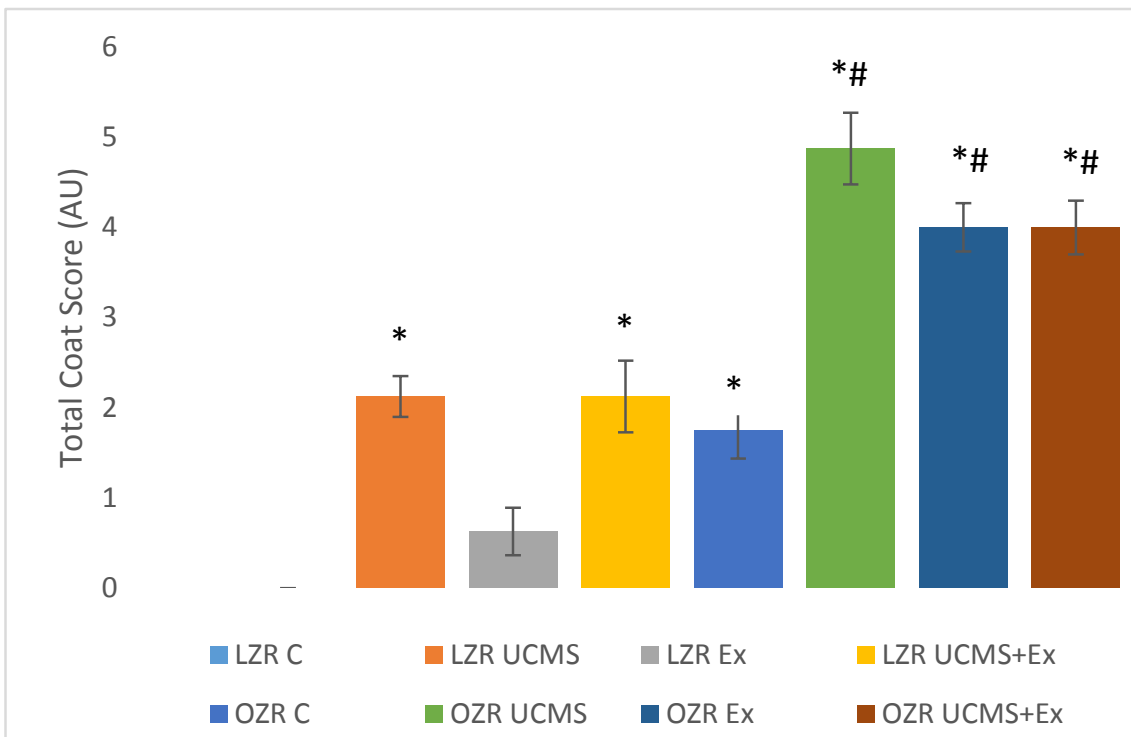

Figure 2. Coat scores. Lean Zucker rat (LZR) control (C) coat scores differed from all groups with the exception of LZR Exercise (Ex). Obese Zucker rat (OZR) C coat scores differed from all groups except LZR unpredictable chronic mild stress (UCMS). * = $p<0.001 . \#=p<0.04 . n=7-8$.

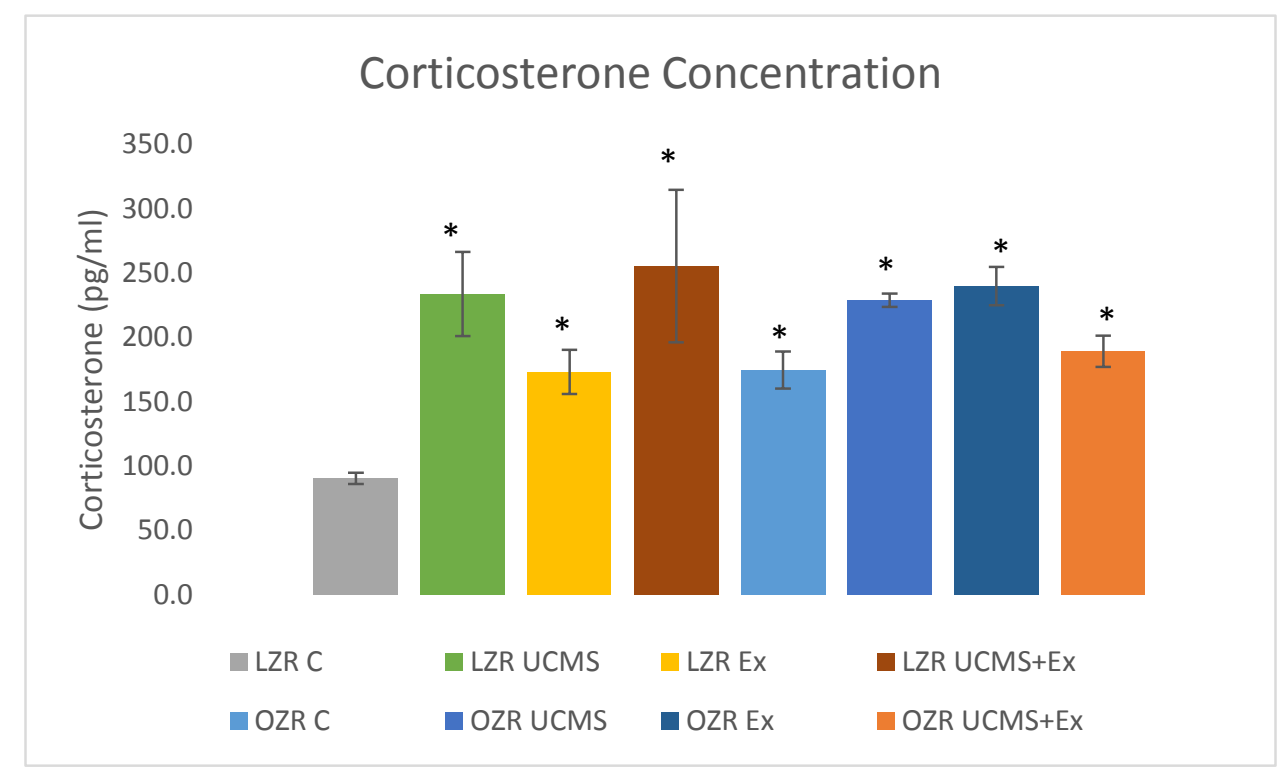

Figure 3. Basal corticosterone concertation. Lean Zucker rat (LZR) control (C) corticosterone concentration was found to be significantly less than all other groups. In LZR animals unpredictable chronic mild stress (UCMS) caused a trend towards an increase in corticosterone, regardless of exercise (Ex) training. In obese Zucker rat (OZR) animals UCMS and Ex both increased corticosterone, with the combination of UCMS+Ex showing an apparent decrease in corticosterone. $\mathrm{P}<0.05$ vs. LZR C. $n=3$ for all groups. 


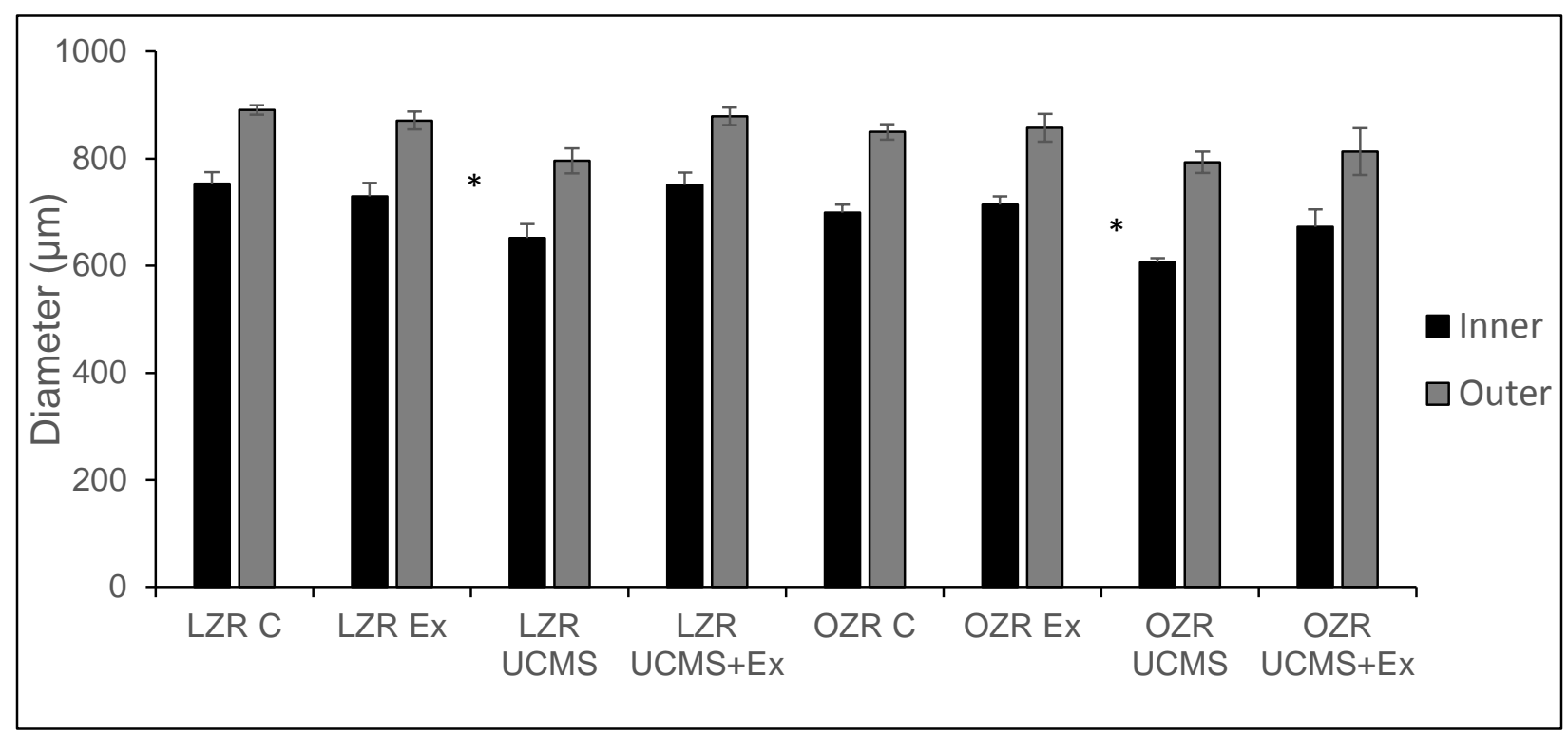

Figure 4. Carotid artery diameter at a mean arterial pressure (MAP) of $100 \mathrm{mmHg}$. Unpredictable chronic mild stress (UCMS) was shown to cause decreases in inner diameter, with exercise $(E x)$ training recovering inner diameter. No differences were found in outer diameter. Values: mean \pm SEM. ${ }^{*}=p<0.05$ vs. LZR C. $n=3-7$

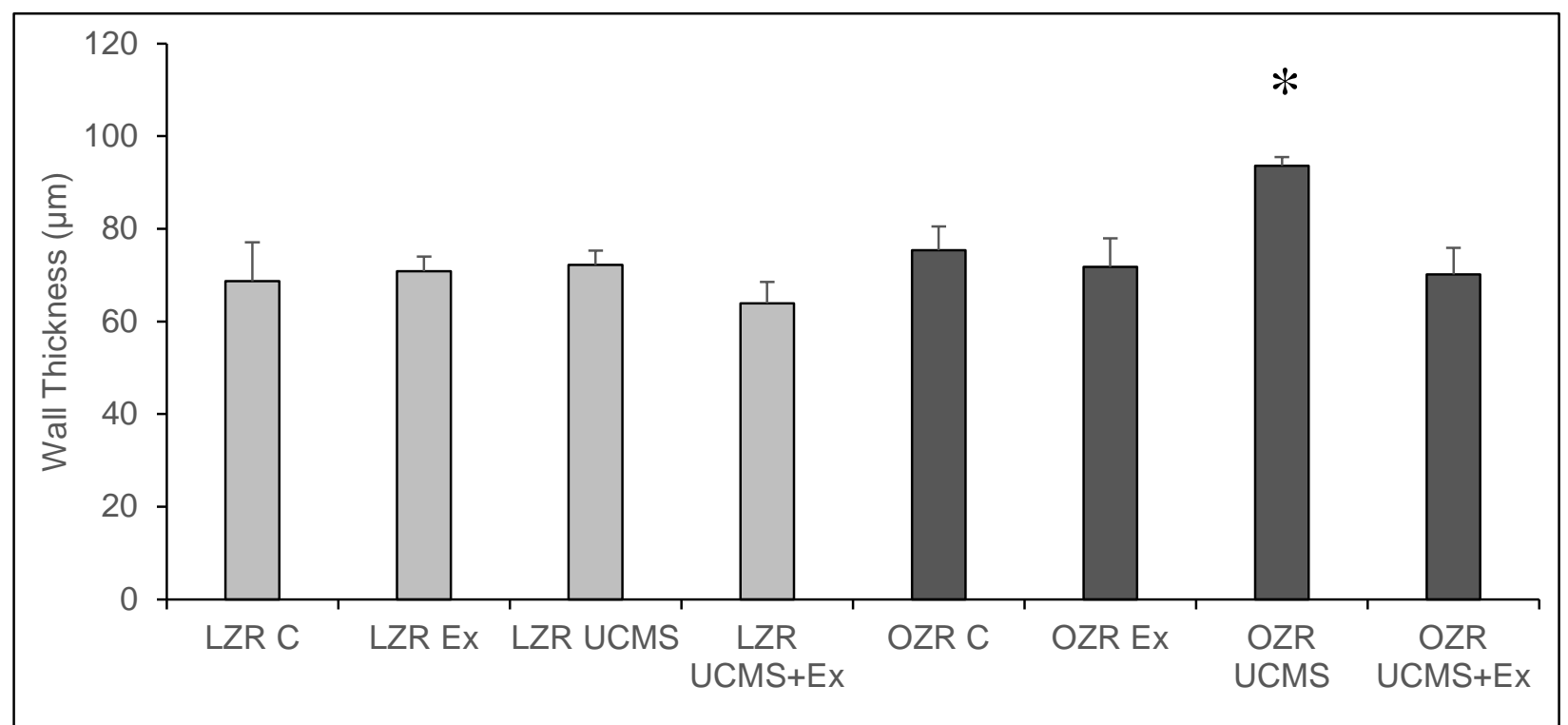

Figure 5. Carotid artery wall thickness (WT) at a mean arterial pressure (MAP) of $100 \mathrm{mmHg}$. Unpredictable chronic mild stress (UCMS) and Metabolic Syndrome (MetS) were shown to cause an increase in WT. Exercise (Ex) training was able to attenuate thickening walls. Values: mean \pm SEM. $=p<0.04$ vs. LZR C. $n=3-6$. 


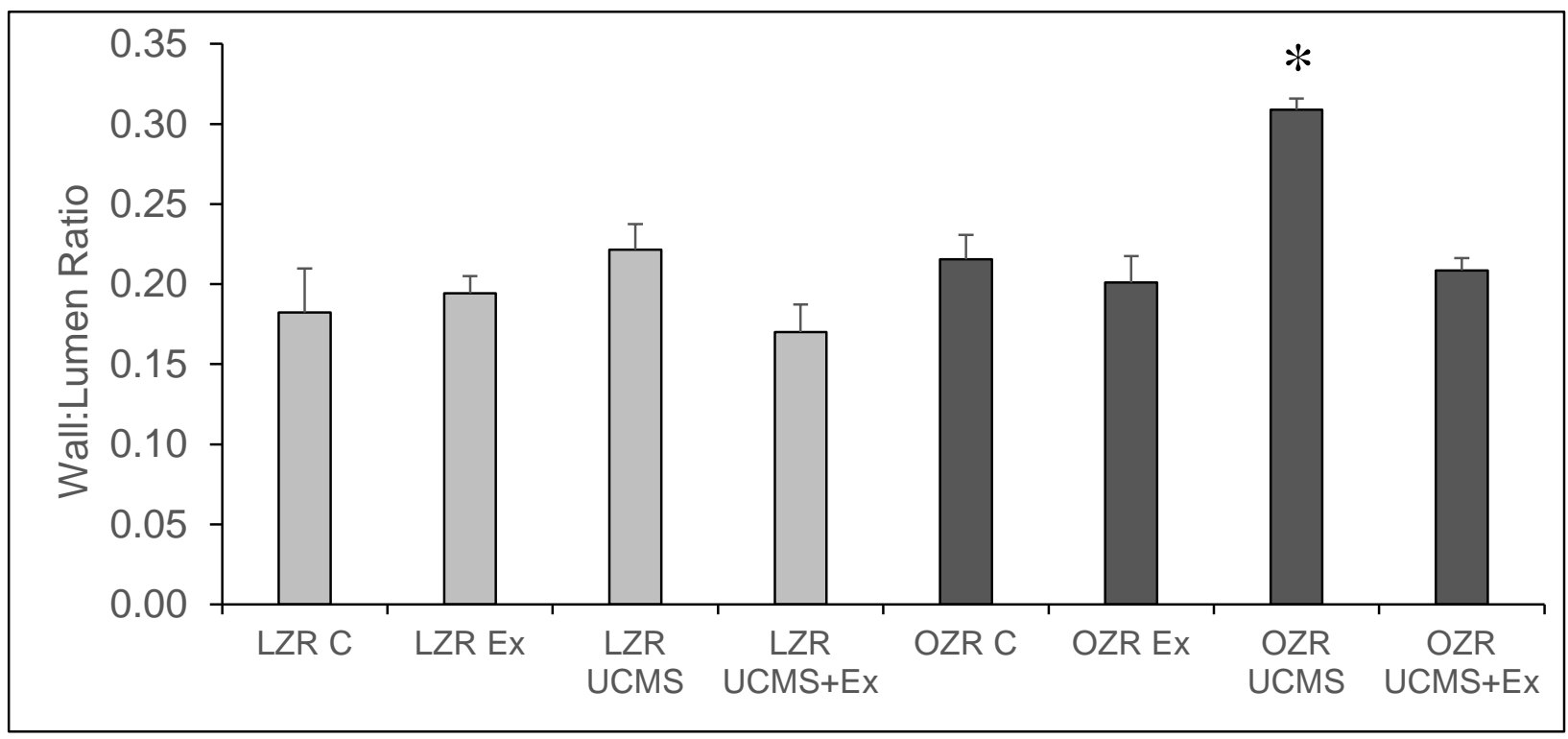

Figure 6. Carotid artery wall:lumen ratio (WLR) at a mean arterial pressure (MAP) of $100 \mathrm{mmHg}$. Unpredictable chronic mild stress (UCMS) and Metabolic Syndrome (MetS) were shown to cause an increase in WLR. Exercise (Ex) training was able to decrease WLR Values: mean \pm SEM. * $=p<0.009$ vs. OZR C. $n=3-6$

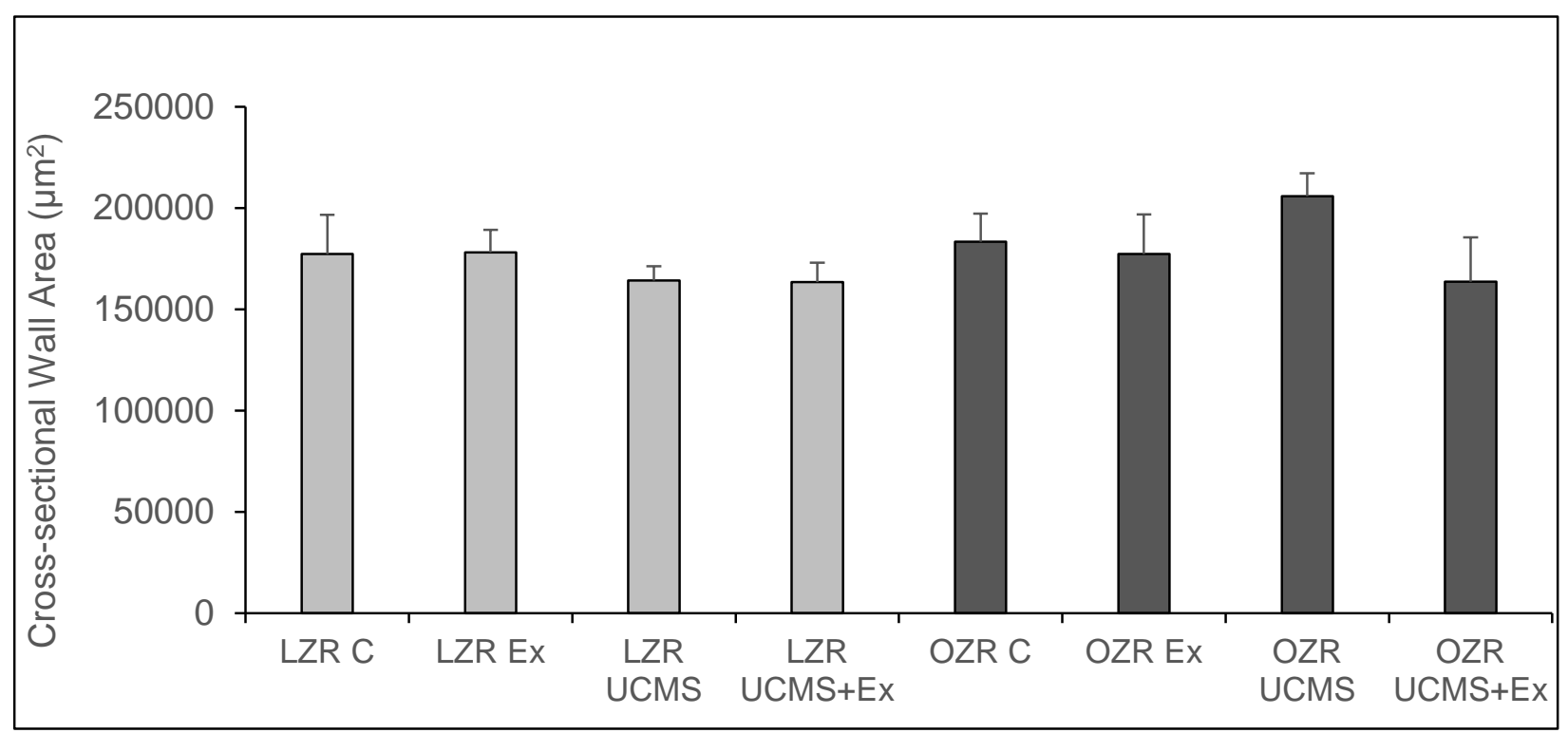

Figure 7. Carotid artery cross-sectional wall area (CSWA) at a mean arterial pressure $M A P)$ of $100 \mathrm{mmHg}$. Values: mean \pm SEM. No statistical differences were found. 


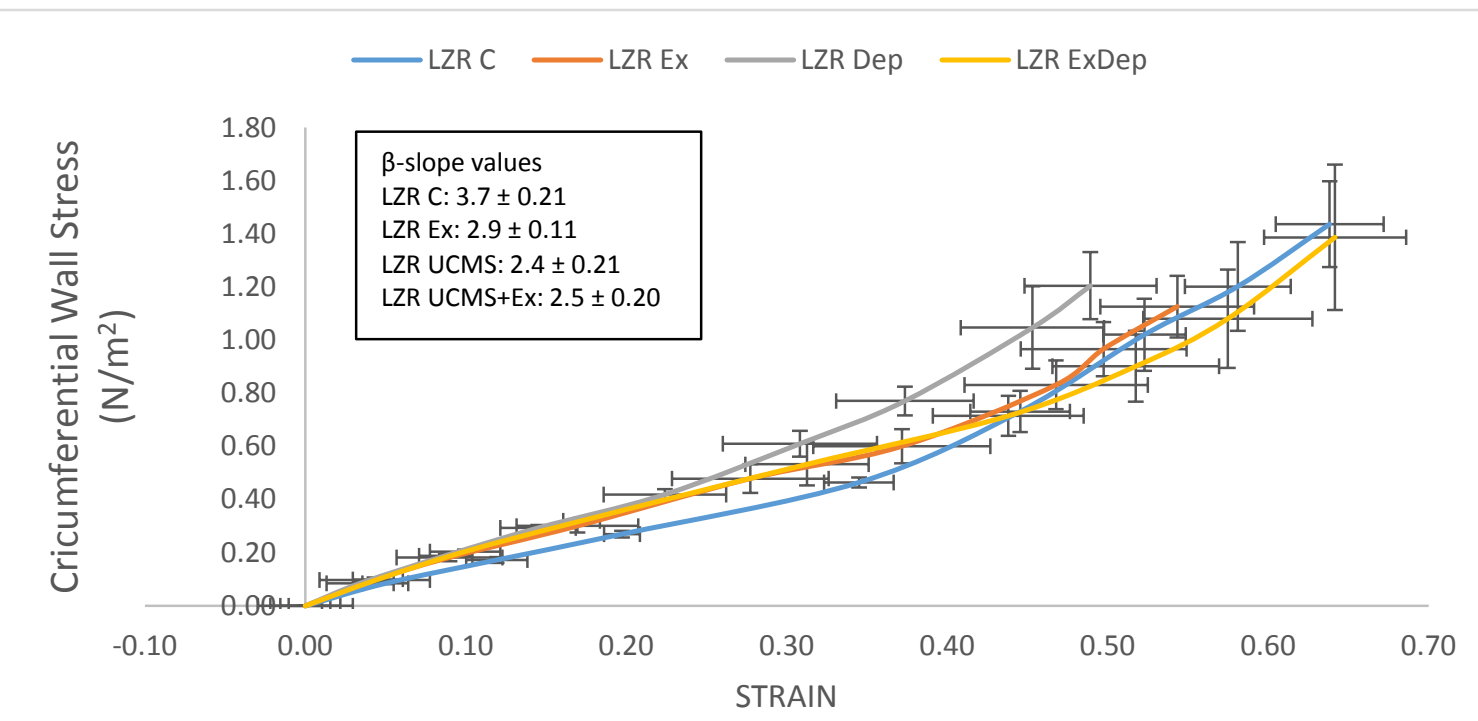

Figure 8. Carotid artery stress-strain for lean Zucker rat (LZR) animals from 0-160mmHg. Unpredictable chronic mild stress (UCMS) was shown to trend towards a stiffer, less compliant vessel. Values: mean \pm SEM. No statistical differences among $\beta$-slopes were found.

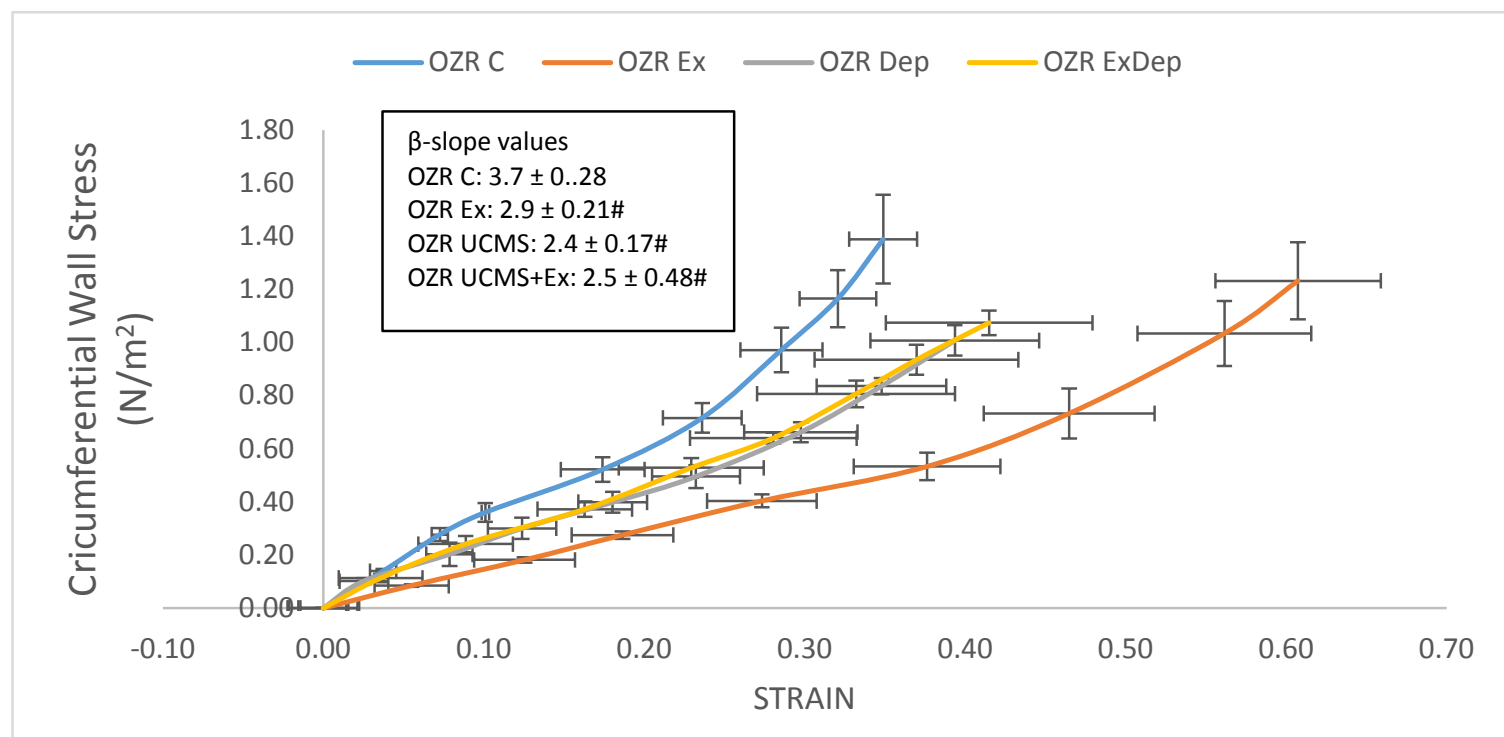

Figure 9. Carotid artery stress-strain for obese Zucker rat (OZR) animals from 0-160 $\mathrm{mmHg}$. Exercise (Ex) improved the stress-strain relationship in OZR animals. Unpredictable chronic mild stress (UCMS), Ex, and UCMS+Ex were all found to significantly improve $\beta$-slope values compared to control. Values: mean \pm SEM. $\# p<0.05$ to OZR C. 


\section{Discussion}

The comorbid state of MetS and depression is one of great complexity and growing prevalence. Our model, utilizing the OZR and UCMS protocol, provides a controlled parallel to a human suffering from these two afflictions. In our study we observed significant weight loss in all LZR cohorts when compared to control. However, OZR animals did not show the same results, with OZR UCMS animals being the only group to show a significant decrease from OZR C. We speculate the lack of weight loss in the two OZR exercise-trained groups can be attributed to an increase in muscle mass, resulting in increased weight. However, muscle samples were not taken from these animals, rendering our data to be speculative only (Table 1).

Insulin and glucose are common parameters measured to verify MetS. However, as our rats were not fasted we elected not to include non-fasting glucose data. Additionally, the laboratory used to determine insulin levels did not have sensitive enough equipment to quantify insulin in our rat blood. Although these two markers are common and important in determining MetS we remain confident in the OZR as a suitable model of MetS based on previous literature (Stanley, et al., 2014; Frisbee, et al., 2006; Zucker, et al., 1972).

As anticipated OZR animals aid significantly more food than LZR animals (Table 1). The alteration to the OZR's leptin receptor results in an inability to gain satiety and mass food consumption (Zucker, 1972). As a result these animals exhibit markedly increased food intake and weight gain. However, the UCMS protocol has been shown to decrease body weight in rodents (Willner, 1994). These results were confirmed by our 
study, as UCMS animals observed an increase in weight loss, paired with an increase in food consumption, indicating the UCMS of inducing changes in metabolism.

We observed an increase in work in OZR UCMS+Ex animals and a decrease in work in LZR UCM+Ex animals (Figure 1). These results indicate our method of matching work to be inaccurate. However, exercise training was able to show significant improvements or trends toward vascular improvement in to UCMS+Ex groups when compared to their respective controls. Given these results we remain confident in our exercise training model as being viable in this study.

No differences were observed among LZR cohorts compared to LZR C or OZR cohorts compared to OZR C. OZR animals were shown to have higher MAP than LZR C, however only OZR C and UCMS groups were significantly different. This implies exercise training was able to trend towards an improvement in MAP in OZR animals (Table 1). Eight weeks of exercise training has been shown to be ineffective in significantly altering blood pressure in humans, indicating our lack of robust MAP changes to be similar to occurrences in a human study (Donley, et al., 2014).

Coat scores and corticosterone concentrations were used to quantify stress in rodents (Figures 2 and 3). Corticosterone ELISA showed LZR C to have markedly decreased corticosterone concentration compared to all other groups. UCMS was effective in increasing corticosterone concentration in LZR animals. Exercise training also increased corticosterone concertation in LZR animals. OZR animals showed a marked increase in corticosterone levels, with UCMS and Ex both increasing corticosterone above control levels. Corticosterone concentrations fluctuate diurnally in rodents, resulting in time of blood draw being extremely important when determining 
corticosterone levels (Weitzman, et al., 1971). Blood samples were taken at different times throughout the day, making it difficult to rely on corticosterone as an accurate measure of stress.

Coat scores were also used as a marker of stress. Coat score data showed LZR C animals to have markedly better scores than all. UCMS and UCMS+Ex in LZR animals increased coat score values significantly. All OZR coat scores were higher than LZR C, but all other OZR groups showed higher scores than OZR C. In the exercise trained animals this is likely due to lesions suffered from the running protocol, due to their fat pads rubbing on the treadmill. When coat score locations OZR animals could not reach (i.e. back and neck) were removed a decrease in coat scores in OZR animals was observed, but scores were still much higher than OZR C. As a result a differentiation should be made in the future between injury and a dirty coat.

To our knowledge this is the first study to evaluate passive structural remodeling of the carotid artery in a model of MetS and depressive-like symptoms. To provide a translatable model for arterial remodeling vessels were pressurized at $100 \mathrm{mmHg}$ and compared. Although robust changes were not observed between LZR cohorts, a trend did appear for UCMS to cause a slightly thicker wall and WLR (Figure 5 and 6, respectively). This observation was exacerbated in OZR animals, with OZR UCMS animals displaying a markedly increased WT and WLR $(p<0.05)$. Although not significant, a trend also appeared in OZR UCMS animals for an increase in CSWA (Figure 7). These increases provide evidence of inward hypertrophic remodeling, as the wall thickness increased and lumen size decreased. Hypertrophic remodeling is associated with decreased vessel compliance, arterial stiffness, hypertension, and vascular dysfunction 
(Intengan, et al., 2001). Exercise training was able to drastically improve WT, WLR, and CSWA in OZR UCMS+Ex animals ( $p<0.05)$. Interestingly, the benefits of exercise were not more robust in the absence of UCMS. These results indicate. LZR UCMS+Ex animals saw a trend towards improvements in WT, WLR, and CSWA, indicating the UCMS model of being capable of blunting the benefit of exercise training in a lean, healthy rodent. These results aid in verifying the effectiveness of the UCMS protocol, as the LZR is a very healthy animal (Stanley, et al., 2014).

The improvements in WLR and WT in OZR UCMS+Ex animals compared to OZR UCMS animals allows for more compliance, flow, dispensability, and overall health. Although WT and WLR improved in OZR UCMS+Ex animals and OZR Ex animals when compared to OZR UCMS animals no significant changes were noted between the two exercise groups. This indicates, from a structural remodeling standpoint, the OZR's ability to improve vessel health with exercise is blunted. It is possible, with the poor metabolic health of the OZR they have a cap on how much benefit exercise training can have on the structural remodeling of the vessel. Paired with the lack of notable weight loss and improvements to MAP in OZR UCMS+Ex and OZR Ex groups compared to OZR C it is also possible our model was not capable to dramatically alter the vascular health through structural remodeling alone. As this is the first study to assess structural remodeling on the carotid artery in a comorbid state of MetS and chronic stress further studies are needed to determine how structural remodeling is impacted in these diseased states and by exercise in the OZR model.

Stress-strain curves for the carotid artery showed a trend for exercise training to improve the stress-strain relationship (Figures 8 and 9). To compare stress-strain curves 
the $\beta$-slope was calculated. $\beta$-slope is a measurement of when the curve begins to take a more linear shape. A more compliant vessel will exhibit a smaller $\beta$-slope value, which was shown in exercised animals. Although LZR animals did not see a significant change among cohorts, all OZR groups were significantly different from OZR C. In agreeance with past literature exercise training dramatically improved $\beta$-slope values (Frisbee, 2006). The combination of Ex+UCMS showed improvement from control, but not the extent of exercise alone. These results suggests the UCMS protocol of blunting the benefits of exercise, while also showing exercise can overcome vascular dysfunction caused by chronic stress. Interestingly, OZR UCMS animals showed improved $\beta$-slope values when compared to OZR C, suggesting stress to be beneficial for the vasculature. A likely explanation is the health of the OZR's vessels is impaired so heavily to begin with the addition of the stress stimulus does not further exacerbate the problem when observing how the vessel responds to increased stress and strain. This yields to the potential explanation for al compensatory mechanism to attempt to preserve the health of the vessel in situations of extreme inflammation, oxidative stress, and immune infiltration (Chantler \& Frisbee., 2014).

Previous studies have shown larger differences between vessel diameters in peripheral resistance arterioles between LZR and OZR animals (Bouvet, et al., 2007; Stepp, et al., 2004). However, less stark changes are observed in the middle cerebral artery (MCA) of LZR and OZR animals (Stepp, et al., 2004). One study showed exercise to be effective in reducing conduit artery WT in obese rats to values similar to lean rats (Pinheiro, et al., 2006). However, another study found habitual exercise to be largely ineffective in altering carotid intima-media thickness in exercising males (Tanaka, et al., 
2002). Our results, although not robust, show aerobic exercise to be capable of improving functional remodeling of the carotid artery in a comorbid state of MetS and chronic stress. 


\title{
Chapter 4:
}

\section{Aerobic Exercise Intervention Improves}

Nitric Oxide-Dependent Processes in a

Rodent Model of Metabolic Syndrome

\author{
and Chronic Stress
}




\begin{abstract}
Metabolic Syndrome (MetS) and chronic stress/depression are associated with an increased risk for poor cardiovascular outcomes and impaired vascular function. Both of these diseases are continuously increasing in prevalence, but not much is known about the pathological effects of their comorbidity. Exercise is a widely accepted and supported modality for combating MetS and managing chronic stress. However, a gap in the literature exists regarding the degree to which exercise can mitigate the vascular dysfunction associated with MetS and chronic stress simultaneously. The Unpredictable Chronic Mild Stress (UCMS) model is used as a simulation of daily, prolonged stress. The Obese Zucker Rat (OZR) represents MetS, with Lean Zucker Rats (LZR) paralleling a healthy individual. PURPOSE: To determine the degree to which exercise can attenuate vascular dysfunction caused by chronic stress and metabolic syndrome. METHODS: OZRs and LZRs were separated into experimental groups including: control (C), exercise (Ex), UCMS, and UCMS with exercise (UCMS+Ex). The aorta was isolated hung on an ex vivo wire tension myograph system, where changes in aortic tension were measured in response to pharmacological agents. Nitric oxide (NO) bioavailability was also measured. RESULTS: Exercise training improved LZR and OZR endothelium-dependent dilation. LZR and OZR UCMS+Ex dilation to methacholine was not different from LZR C and OZR C, respectively. Exercise training improved nitric oxide (NO) bioavailability in OZR and LZR animals. OZR-UCMS NO bioavailability was significantly less compared to all groups. LZR and OZR UCMS+Ex NO bioavailablity were not different from their respective controls. CONCLUSIONS: Comorbidity between MetS and chronic stress is a confounding factor on healthy vasculature, but does not increase vascular dysfunction in already impaired vessels. Exercise can be used as an intervention for these two diseases to improve conduit artery health.
\end{abstract}


Introduction

Metabolic Syndrome (MetS) and depression are both risk factors for cardiovascular disease (CVD), and have been bi-directionally linked to each other (Dunbar, et al., 2008). The increased risk of CVD in MetS (Stehouwer, et al., 2008) and depression (Seldenrijk, et al., 2011) have both been linked to decreases in nitric oxide (NO) production (Frisbee, et al., 2005) and increased inflammation (Shimbo, et al., 2005; Yaffe, et al., 2004), further complicating the relationship between the two diseases and the pathological implications associated with them.

The vascular dysfunction associated with MetS and depression has been shown to be prevalent in the conduit arteries (Schillaci, et al., 2005 \& Stanley, et al., 2014). The conduit, or elastic arteries, are responsible for maintaining a constant pressure throughout the arterial system, and include the carotid arteries and aorta. Alterations to conduit function can result in pathological conditions and alterations in normal cardiac function and blood flow. MetS can result in negative changes to the conduits through decreases in endothelium-dependent vasodilation and increased levels of oxidative stress (Heinonen, et al., 2014).

Moderate intensity aerobic exercise has been shown to have beneficial effects on the vasculature of individuals with MetS (Donley, et al., 2014) and depressive-like symptoms (Kiuchi, et al., 2012). Furthermore, exercise has been shown to be neuroprotective (Teri, et al., 2003; Nabkasorn, et al., 2006) and to have a favorable effect on the majority of risk factors associated with MetS (Pattyn, et al., 2013). Exercise training is linked to increases in endothelial NO synthase (eNOS) and NO activity in the aorta (Cacicedo, et al., 2011). 
To simulate MetS the obese Zucker rat (OZR) model was used. The OZR (fa/fa) exhibits rapid weight gain, hypertension, elevated blood glucose, and dyslipidemia making it a suitable model for MetS (Zucker, 1972). In order to simulate depression-like symptoms the unpredictable chronic mild stress (UCMS) protocol was implemented. The UCMS protocol produces depressive-like symptoms in rodents through decreases in award seeking, increased HPA axis activity, adrenal hypertrophy, and increases in corticosterone production (Willner, 1997).

The goal of this study was to fully assess the structural and molecular changes in the conduit arteries caused by depression and MetS as comorbidities, and evaluate the effectiveness of moderate intensity aerobic exercise on the vasculature of this comorbid state. To simulate MetS the OZR model was used, with the LZR serving as the healthy control. Furthermore, the UCMS protocol was utilized to induce depression-like symptoms in the animals. We hypothesized the comorbid state of MetS and depression would result in decreased endothelium-dependent dilation and decreased NO bioavailability and exercise training would be able to attenuate the vascular dysfunction caused by the comorbidities.

Materials and Methods

Animals and Housing: Male LZR and OZR (Lepr fa/fa)) were purchased from Envigo Labratories (Indianapolis, IN) and housed in a pathogen-free vivarium room with a 12hr:12hr reverse light:dark cycle, in standard Allentown rat cages. 
Feeding and handling: Rats were fed standard rodent chow and tap water ad libitum. Animal handling and care were performed in accordance with the Public Health Service Policy on Humane Care and Use of Laboratory Animals and West Virginia University's Institution of Animal Care and Use Committee.

Study Design: Rodents were divided into eight experimental groups: LZR control (C) $(n=8)$, LZR exercise $(E X)(n=8)$, LZR UCMS $(n=8)$, LZR EX+UCMS $(n=8)$, OZR C $(n=8)$, OZR EX $(n=8)$, OZR UCMS $(n=8)$, and OZR UCMS+EX $(n=10)$. Animals were single housed. Rats were weighed and their weights recorded weekly for the duration of the study. To ensure animals were being adequately fed food amount was weighed and recorded twice a week. Control animals remained in the vivarium room and were only disturbed for body and food weighing, and coat scoring throughout the duration of the study.

Coat Scoring: Coat score data was obtained weekly to determine the health and grooming habits of each animals. The total cumulative score was determined by giving an individual score of "0" (clean) or "1" (dirty) to eight different body areas (head, neck, back, underside, tail, hindlimb, forelimb, and genitals).

Veterinary Treatment: If animals sustained injuries or lesions during the course of the study they were reported to WVU's Office of Laboratory Animal Research veterinary staff. The veterinary staff inspected and treated the animal. If further attention was needed directions were given and strictly adhered to. 
Exercise Training: Animals were run on a Columbus Instruments rodent treadmill (Exer3/6) for 60 minutes per day, five days per week, over the course of the eight week study. Animals were run on a slight incline of 10 degrees to place more emphasis on training the fore and hindlimb muscles. On the first day of training animals were placed on the treadmill for 10 minutes, running at $5 \mathrm{~m} / \mathrm{min}$. During the first week, each day time and speed were increased, until at the start of week two animals were exercising for 60 minutes, reaching a speed of $20 \mathrm{~m} / \mathrm{min}$ (LZR) or $14 \mathrm{~m} / \mathrm{min}(\mathrm{OZR})$. Animals exercised on a progressive exercise regimen, beginning each session with a 5 minute warm-up at 5 $\mathrm{m} / \mathrm{min}(\mathrm{OZR}$ ) or $7 \mathrm{~m} / \mathrm{min}$ (LZR) and progressively increasing speed throughout the session. If an animal refused to run, by sitting on the shock bay, resisting gentle prodding, the shock bay was turned off and a note was made of the animal's refusal to run.

Maximal Speed Test: To determine if a training effect was present a maximal speed test was performed during weeks one and eight. Animals began running at $5 \mathrm{~m} / \mathrm{min}$ with speed increasing by $2 \mathrm{~m} / \mathrm{min}$ every 30 seconds until exhaustion. Exhaustion was defined as when the rat sat on the shock bay with no attempts to continue exercising for 5 seconds. Maximal running speeds were recorded following exhaustion.

Work Calculation: Work $(J)$ was calculated in an attempt to normalize speed and body weight across all experimental groups. Work was calculated using the equation $(B M * 9.81) *\left(\sin \frac{\theta * \pi}{180}\right) * D$, where BM is body mass $(\mathrm{kg}), 9.81$ is gravity, $\theta$ is the angle of the treadmill, and $D$ is the distance run (m). 
Unpredictable Chronic Mild Stress (UCMS) Protocol: The UCMS protocol was used to simulate chronic stress/depression. Rodents were subjected to a variety of stresses over a 6-8 hour period five days per week. Stresses included: the removal of bedding (no bedding), the addition of $10 \mathrm{oz}$. of water to the bedding (damp bedding), the removal of bedding and the addition of $10 \mathrm{oz}$. of water to the cage (bath), the removal of bedding and a 45 degree cage tilt (cage tilt), relocation of rodents into another rodents cage (social stress), and successive light/dark cycles (light/dark cycle). Each stress was performed for 2-4 hours per day so animals were exposed to 2-3 stresses per day. The stresses were designed to be unpredictable to prevent acclimation to the protocol, therefore stresses were purposefully different each day with the protocol never repeating on consecutive days.

Injection, Tracheotomy, Mean Arterial Pressure, and Blood Draw: Animals were injected with sodium pentobarbital (Nembutal $50 \mathrm{mg} / \mathrm{kg}$ ) via intraperitoneal injection. Animals were inspected for consciousness via toe pinch. Booster injections were given until the rodent had no response to a substantial squeezing on the right foot with forceps. At the point of unconsciousness the animal's neck was cleaned of fur, skin, fat, muscle, and fascia until the trachea was exposed. A small incision was made in anterior wall of the trachea and a breathing tube was inserted and sutured in place. The right carotid artery was then exposed and cannulated to determine mean arterial pressure (MAP). Following a 5 minute equilibration period the MAP was recorded. Immediately following the recording of MAP an incision was made into the abdomen on the animal and the diaphragm was 
cut. A blood draw was then made from the inferior vena cava, which was immediately centrifuged and taken for lab analysis.

Aorta Removal, Cleaning, Hanging, and Reactivity: The thoracic and abdominal aorta was removed encased in perivascular adipose tissue and placed in PSS. The PVAT was carefully removed, leaving only the intact aorta. The vessel was then sectioned into $3 \mathrm{~mm}$ rings. Each ring was mounted to an ex vivo wire tension myograph system. Force transduction was used to measure the changes in aortic tension in response to pharmacological agonists. The aortic rings were mechanically set to 1 gram of tension then pre-constricted using phenylephrine $(\mathrm{PE})\left(1 \times 10^{-6} \mu \mathrm{M}\right)$, followed by a gradual dilation induced by increasing concentrations of methacholine (MCh) from $1 \times 10^{-9}$ to $1 \times 10^{-5} \mu \mathrm{M}$. Excess sections of the aorta were placed in microcentrifuge tubes and stored at $-80^{\circ} \mathrm{C}$ for analysis later.

Nitric Oxide Bioavailability: NO bioavailability was assessed using a DAF-FM diacetate assay. Two $\mathrm{mm}$ rings of thoracic aorta were placed into a 96 well plate with $100 \mu \mathrm{L}$ of 5 $\mu \mathrm{M}$ DAF-Assay. Rings incubated in assay for 20 minutes at $37^{\circ} \mathrm{C}$. Next rings were stimulated with methacholine (+/- methacholine used to demonstrate eNOS coupling) at a concentration of $1 \times 10^{-5} \mu \mathrm{M}$ or left untreated (equal addition of physiological solution) for 15 minutes. After 15 minutes the plate was removed and placed in an empty well and run in a Biotek synergy HT plate reader, excited at $485 \mathrm{~nm}$ wavelength, and read at 530 $\mathrm{nm}$. Fluorescence is expressed in arbitrary units (AU) normalized to background assay fluorescence and tissue weight. 
Corticosterone ELISA: The concentration of corticosterone in the plasma was measured with an ELISA kit (Cayman Scientific). The change in color was monitored at a wavelength of $405 \mathrm{~nm}$ using a Dynex MRX plate reader. Measurements were performed in duplicate with all comparisons performed with the same assay. The corticosterone content was expressed as $\mathrm{pg} / \mathrm{ml}$ and reported as relative to LZR control values.

Statistical Analyses: All values are expressed as mean \pm SEM unless otherwise specified. A one-way ANOVA was used to compare groups unless otherwise specified. When a main effect was observed a Tukey post-hoc test was performed to determine group differences. All statistics were performed using SPSS.

Results

Body Weight: Body weight increased for all animals over the duration of study. LZR body weight remained consistent, with animals reaching roughly 400 grams, regardless of treatment. OZR gained weight more rapidly, with controls and exercise rodents reaching around $600 \mathrm{~g}$ on average. The OZR UCMS group displayed a similar weight gain, averaging nearly $585 \mathrm{~g}$ by the end of the eight weeks. OZR UCMS+Ex animals displayed a marked decrease in body weight compared to OZR C, averaging nearly $550 \mathrm{~g}$ (Table 1).

Food Intake: OZR C was found to consume significantly more food than LZR C $(p<0.01)$. LZR UCMS consumed less food than OZR UCMS ( $p<0.01)$. LZR Ex animals consumed 
less food than OZR Ex $(p<0.01)$. OZR Ex animals consumed less than OZR UCMS animals $(p<0.01)$. LZR UCMS+Ex consumed less than OZR UCMS+Ex (Table 1).

Work: LZR UCMS+Ex animals performed the least amount of work $(p<0.01)$. LZR Ex and OZR Ex performed the same amount of work. OZR UCMS+Ex performed significantly more work than all groups $(p<0.01)($ Figure 1$)$.

Mean Arterial Pressure: OZR C had increased MAP compared to LZR C. No statistical differences were found between UCMS groups or Ex groups. OZR UCMS+Ex animals were found to have decreased MAP compared to OZR C (Table 1).

Coat Scores: LZR C animals had markedly better coat scores than all OZR groups, as well as LZR UCMS and UCMS+Ex ( $p<0.002)$. Additionally, LZR Ex animals had improved coat scores compared to the LZR groups exposed to UCMS and all OZR groups $(p<0.04)$. OZR C coat scores were significantly better than LZR UCMS+Ex and all other OZR Groups $(p<0.04)$ (Figure 2).

Corticosterone ELISA: LZR C animals showed markedly decrease corticosterone concentrations than all other groups, with LZR Ex having a decrease in corticosterone when compared to LZR UCMS and UCMS+Ex $(p<0.05)$. OZR $C$ had increased corticosterone compared to other OZR groups $(p<0.05)$, with OZR UCMS+Ex showing a decrease in corticosterone compared to OZR Ex and OZR UCMS (Figure 3). 
Aortic Constriction to Phenylephrine: LZR Ex maximal constriction to PE $\left(1 \times 10^{-6} \mu \mathrm{M}\right)$ was significantly less than all other groups. No other statistical differences were present. A trend for UCMS groups to constrict more than their respective control groups appeared to be present (Figure 9).

Aortic Dilation to Methacholine: LZR Ex maximal dilation to MCh $\left(1 \times 10^{-5} \mu \mathrm{M}\right)$ was significantly increased compared to all groups. LZR UCMS dilation was significantly decreased compared to LZR C. LZR UCMS+Ex dilation was significantly increased compared to OZR C, but was not significantly different than LZR C. OZR C dilation was significantly reduced when compared to LZR C. OZR Ex was significantly different from OZR C, but not LZR C. OZR UCMS had a significant decreased in dilation when compared to LZR C. OZR UCMS+Ex was not significantly different from OZR UCMS or OZR C (Figure 10).

Stimulated Nitric Oxide Bioavailability: LZR Ex NO bioavilabiltiy was significantly increased compared to all groups, with LZR UCMS showing a significant decrease in bioavailability compared to LZR C. LZR UCMS+Ex was not significantly different from LZR C. OZR C displayed a significant reduction to LZR C in NO bioavailability. OZR Ex showed a significant increase in NO bioavailability compared to OZR C, with no significant difference from LZR C. OZR UCMS NO bioavailability was greatly diminished, being significantly reduced compared to all groups. OZR UCMS+Ex was no significantly different from OZR or LZR C groups (Figure 12). 
Tables and Figures

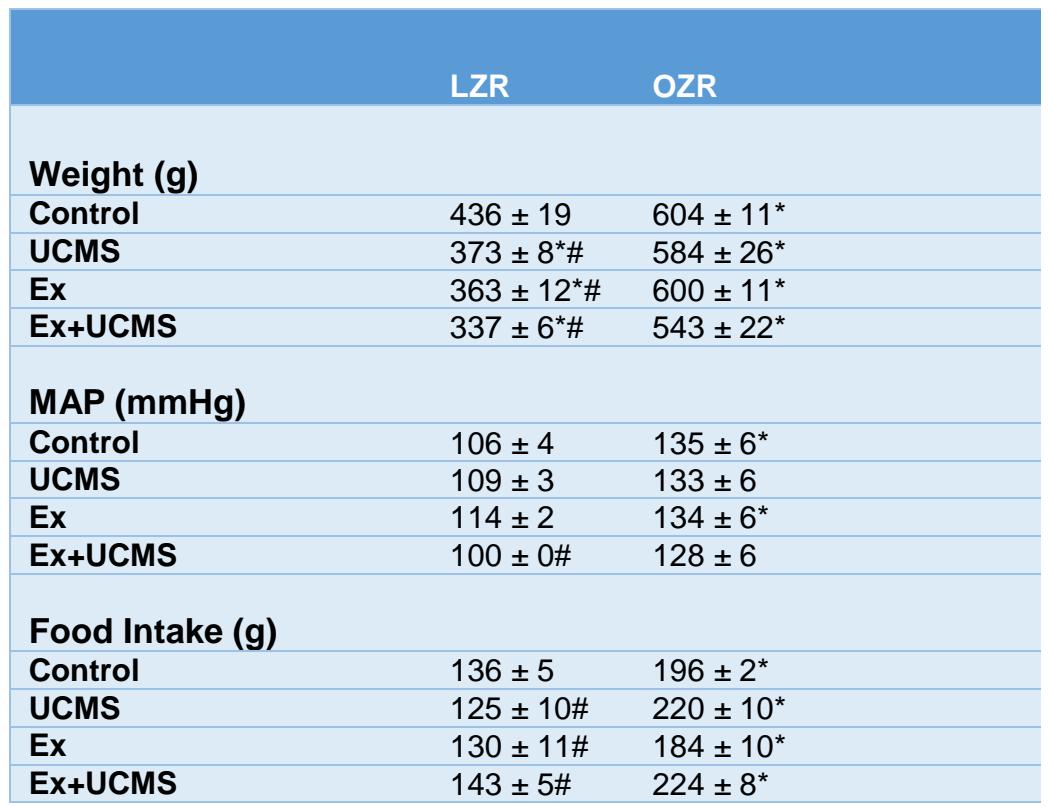

Table 1. Average body weight, mean arterial pressure (MAP), and food intake for all experimental groups. Lean Zucker rat (LZR) control (C) weighed less than all obese Zucker rat (OZR) groups and all other groups. All LZR groups weighed less than OZR C. All LZR groups had significantly lower mean arterial pressure (MAP) than OZR C. MAP did not significantly decrease from OZR $C$ in any OZR group. OZR C consumed more food than all LZR groups, but did not differ significantly from other OZR groups. ${ }^{*}=p$ $<0.05$ vs. LZR C. $\#=p<0.05$ vs. OZR C. $n=8$ for all groups.

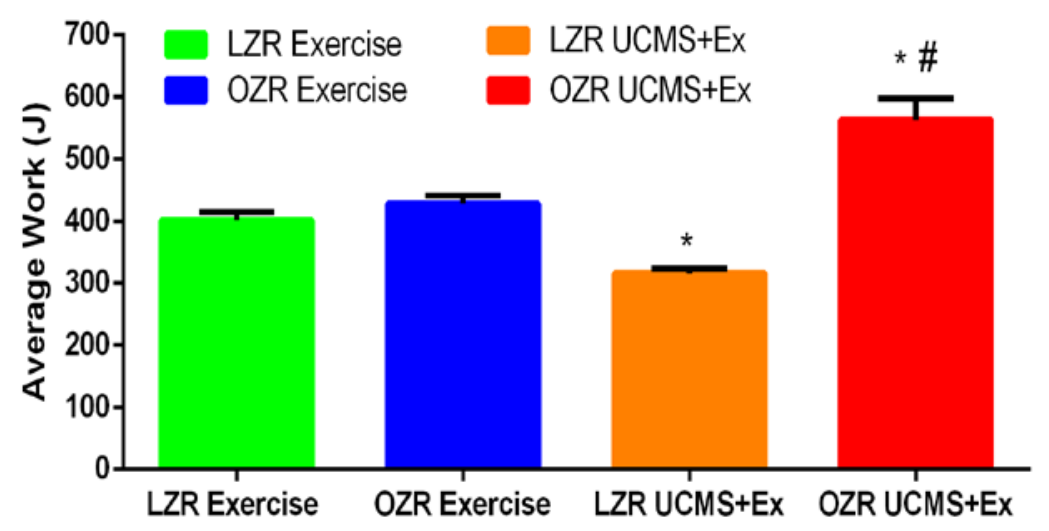

Figure 1. Average work (Joules) for each exercise treatment group. Lean Zucker rat (LZR) and obese Zucker rat (OZR) exercise performed the same amount of work during their exercise training. LZR unpredictable chronic mild stress and exercise (UCMS+Ex) performed the least amount of work compared to all groups. OZR UCMS+Ex had the highest average work. Values: mean \pm SEM. ${ }^{*}=p<0.01$ vs. LZR control; $=p<0.01$ vs. OZR control; $\#=p<0.01$ vs. LZR/OZR in treatment group. All groups $n=8$. 


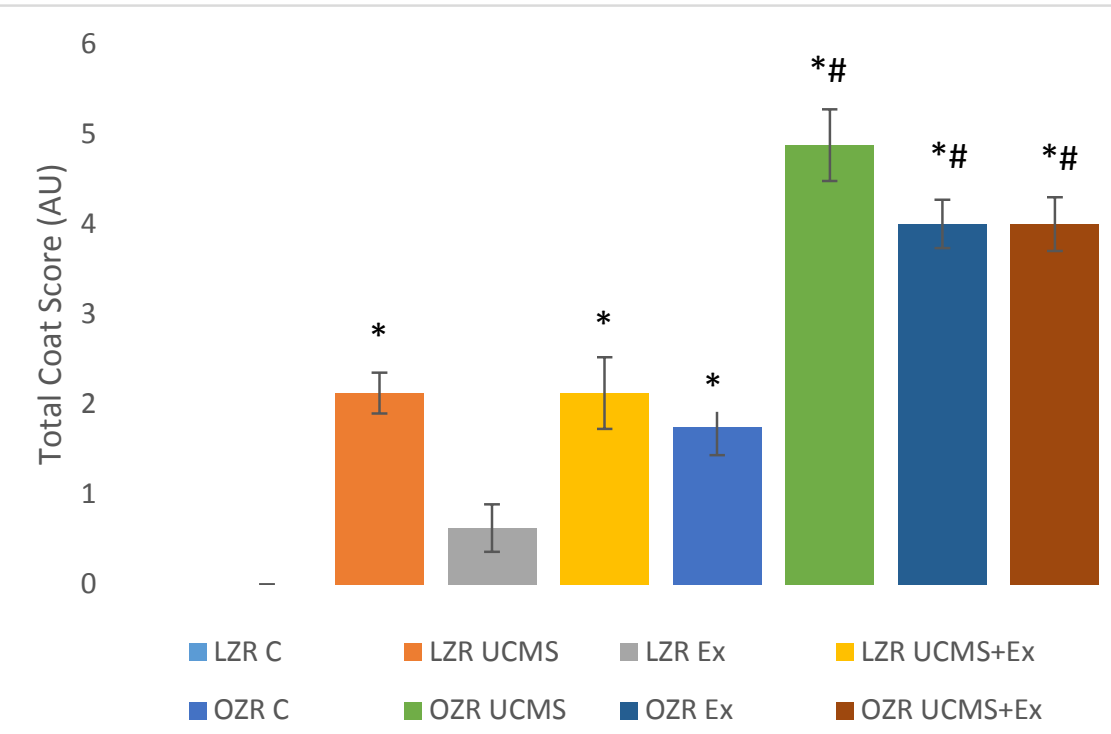

Figure 2. Coat scores. Lean Zucker rat (LZR) control (C) coat scores differed from all groups with the exception of LZR Exercise (Ex). Obese Zucker rat (OZR) C coat scores differed from all groups except LZR unpredictable chronic mild stress (UCMS). * = $p<0.001 . \#=p<0.04 . n=7-8$.

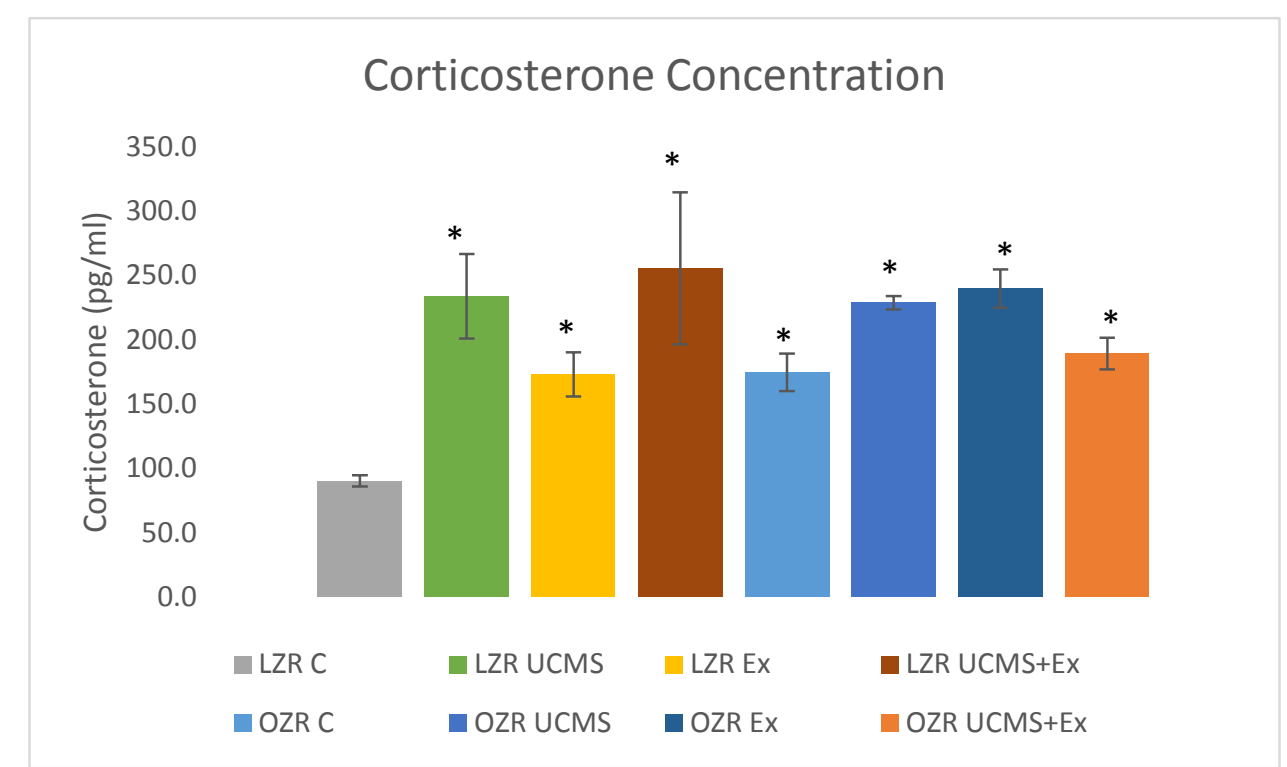

Figure 3. Basal corticosterone concertation. Lean Zucker rat (LZR) control (C) corticosterone concentration was found to be significantly less than all other groups. In LZR animals unpredictable chronic mild stress (UCMS) caused a trend towards an increase in corticosterone, regardless of exercise (Ex) training. In obese Zucker rat (OZR) animals UCMS and Ex both increased corticosterone, with the combination of UCMS+EX showing an apparent decrease in corticosterone. $P<0.05$ vs. LZR C. $n=3$ for all groups. 


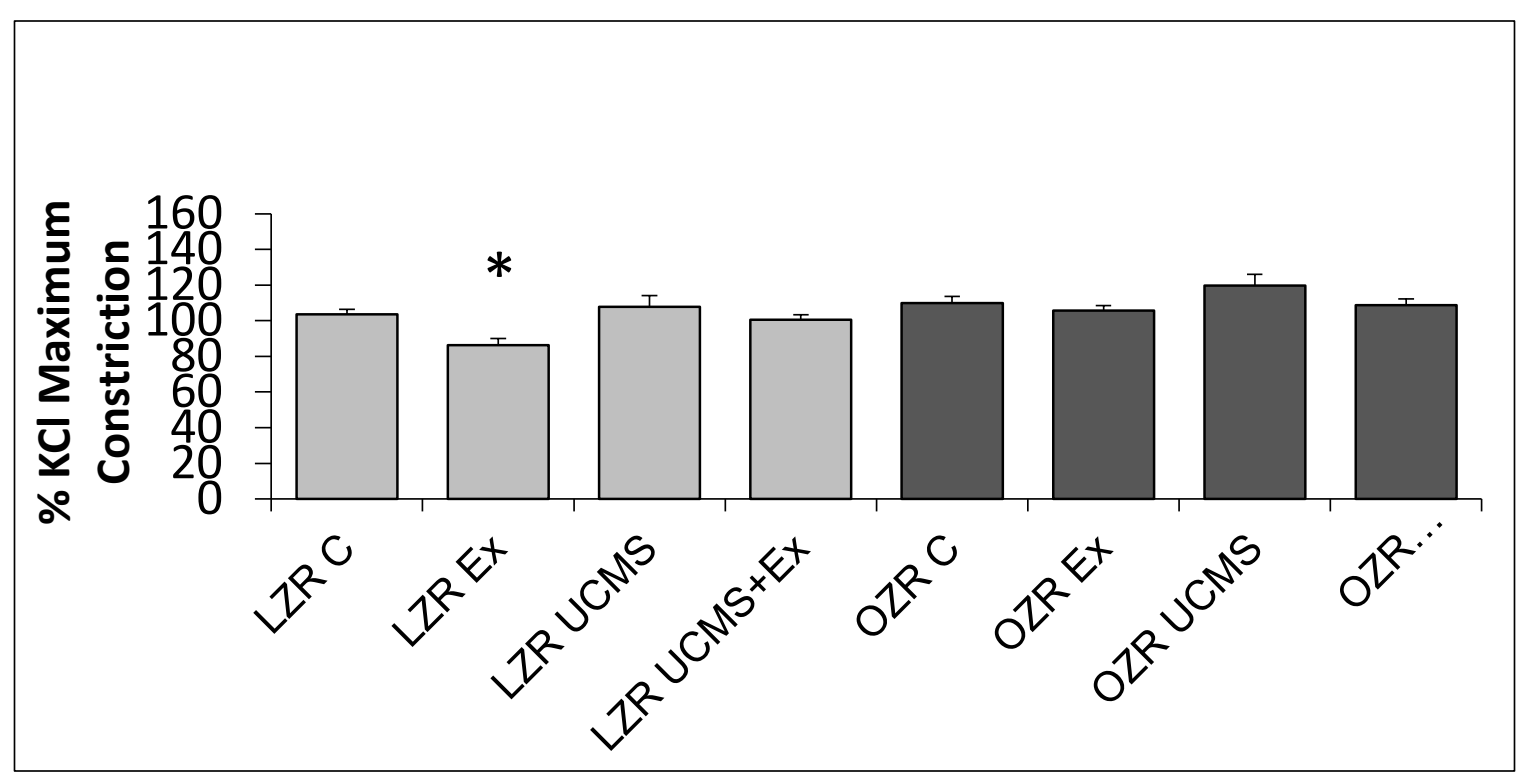

Figure 10. Aortic maximal constriction to phenylephrine. Exercise training (Ex) was able to decrease maximal constriction in lean Zucker rat (LZR) animals. Unpredictable chronic mild stress (UCMS) did not have a significant impact. Values: mean \pm SEM. ${ }^{*}=p<0.05$ vs. LZR C. $n=8$

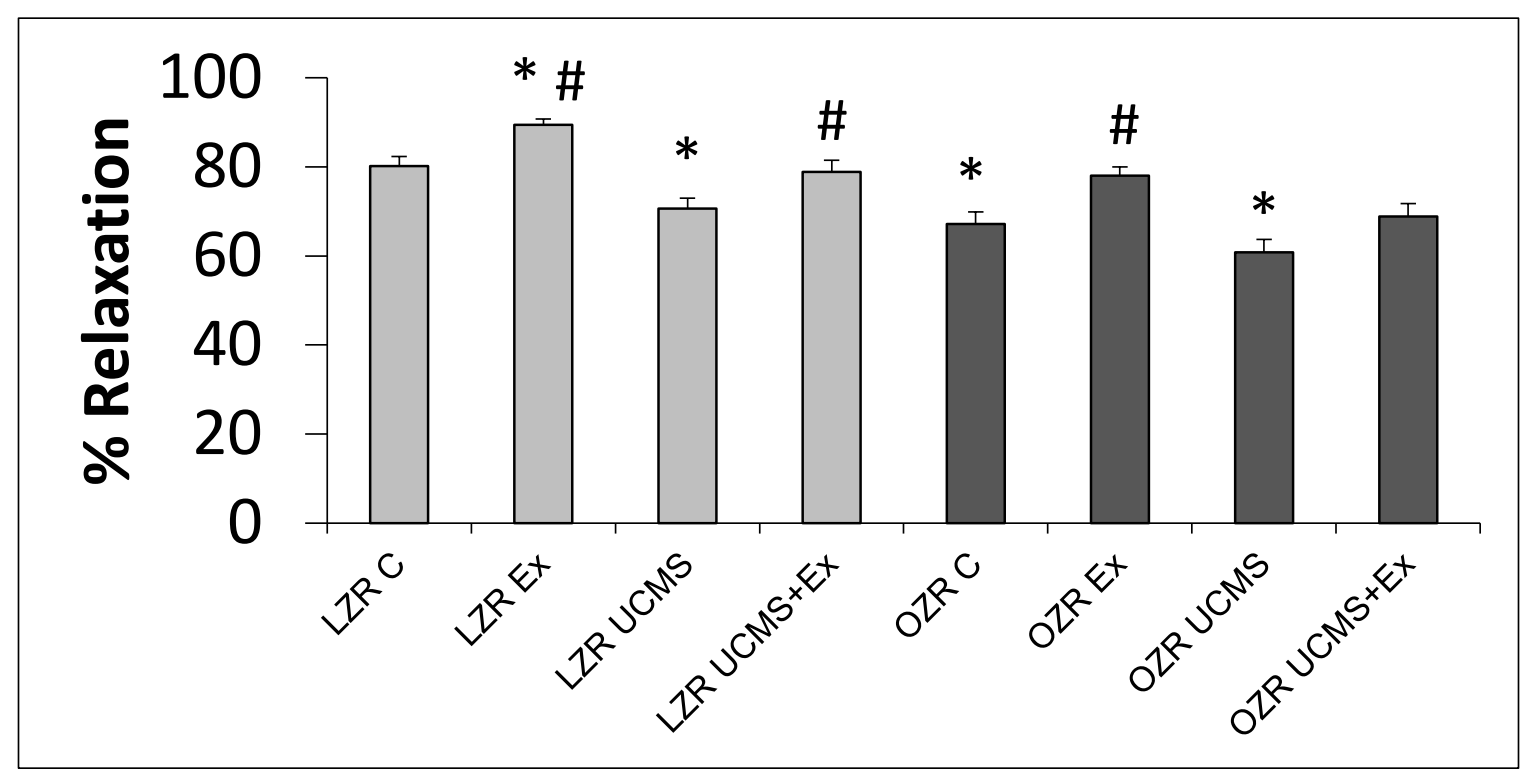

Figure 11. Aortic maximal dilation to Methacholine. Exercise (Ex) training was able to increase dilation in lean Zucker rat (LZR) and obese Zucker rat (OZR) animals. Unpredictable chronic mild stress (UCMS) decreased dilation compared to controls (C). Ex was able to recover dilation in UCMS animals. Values: mean \pm SEM. ${ }^{*}=p<0.05$ to LZR C. \# $=p<0.05$ to OZR C. 


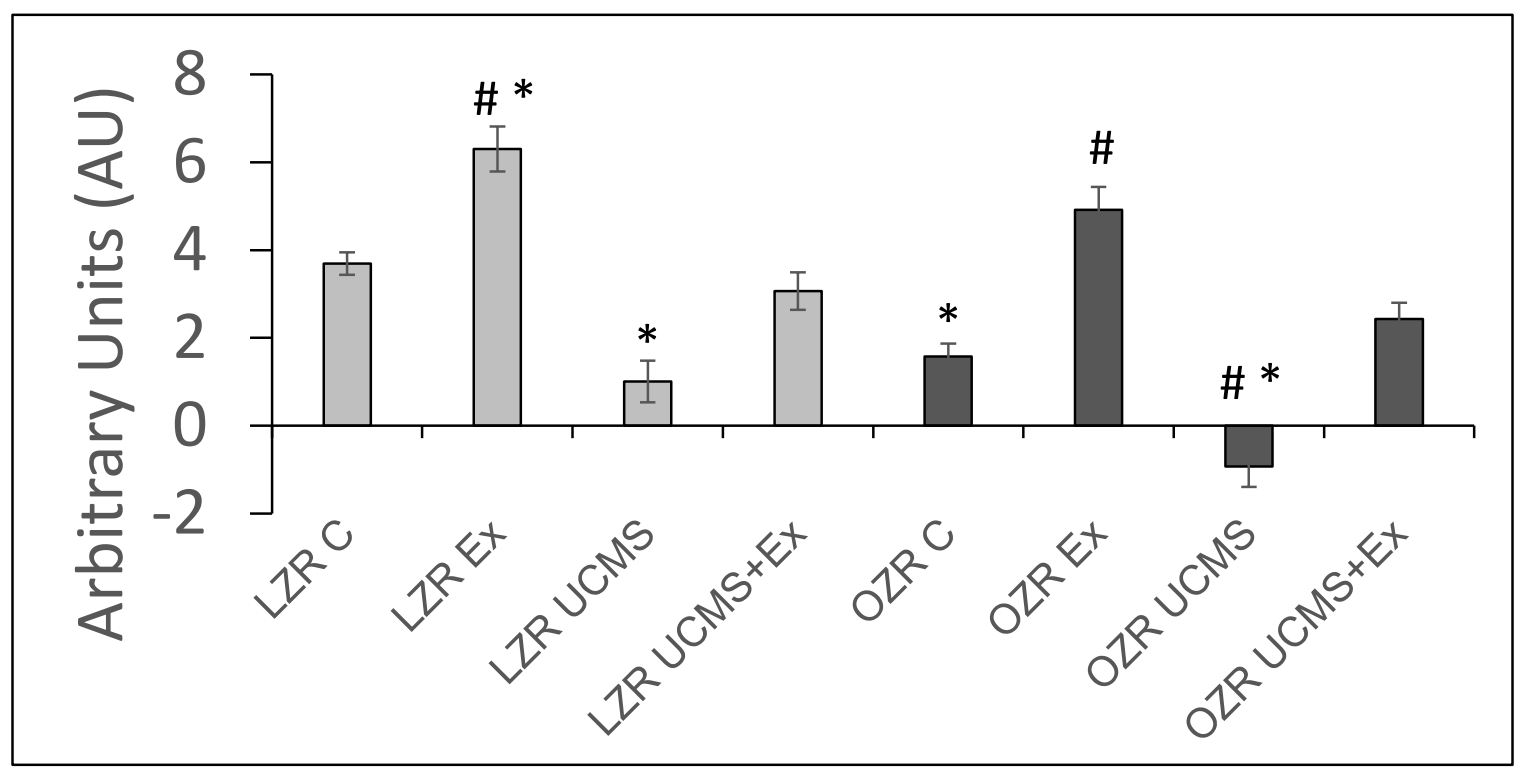

Figure 12. Nitric oxide (NO) bioavailability of the thoracic aorta. Unpredictable chronic mild stress (UCMS) reduced bioavailability from control in both lean Zucker rats (LZR) and obese Zucker rats (OZR). Exercise (Ex) increased bioavailability compared to controls. Ex was able to rescue diminished bioavailability caused by UCMS. Values: mean \pm SEM. ${ }^{*}=p<0.05$ to LZR C. $\#=p<0.05$ to OZR C.

\section{Discussion}

The comorbid state of MetS and depression is one of great complexity and growing prevalence. Our model, utilizing the OZR and UCMS protocol, provides a controlled parallel to a human suffering from these two afflictions. In our study we observed significant weight loss in all LZR cohorts when compared to control. However, OZR animals did not show the same results, with OZR UCMS animals being the only group to show a significant decrease from OZR C. We speculate the lack of weight loss in the two OZR exercise-trained groups can be attributed to an increase in muscle mass, resulting 
in increased weight. However, muscle samples were not taken from these animals, rendering our data to be speculative only (Table 1).

Insulin and glucose are common parameters measured to verify MetS. However, as our rats were not fasted we elected not to include non-fasting glucose data. Additionally, the laboratory used to determine insulin levels did not have sensitive enough equipment to quantify insulin in our rat blood. Although these two markers are common and important in determining MetS we remain confident in the OZR as a suitable model of MetS based on previous literature (Stanley, et al., 2014; Frisbee, et al., 2006; Zucker, et al., 1972).

As anticipated OZR animals aid significantly more food than LZR animals (Table 1). The alteration to the OZR's leptin receptor results in an inability to gain satiety and mass food consumption (Zucker, 1972). As a result these animals exhibit markedly increased food intake and weight gain. However, the UCMS protocol has been shown to decrease body weight in rodents (Willner, 1994). These results were confirmed by our study, as UCMS animals observed an increase in weight loss, paired with an increase in food consumption, indicating the UCMS of inducing changes in metabolism.

We observed an increase in work in OZR UCMS+Ex animals and a decrease in work in LZR UCM+Ex animals (Figure 1). These results indicate our method of matching work to be inaccurate. However, exercise training was able to show significant improvements or trends toward vascular improvement in to UCMS+Ex groups when compared to their respective controls. Given these results we remain confident in our exercise training model as being viable in this study. 
No differences were observed among LZR cohorts compared to LZR C or OZR cohorts compared to OZR C. OZR animals were shown to have higher MAP than LZR C, however only OZR C and UCMS groups were significantly different. This implies exercise training was able to trend towards an improvement in MAP in OZR animals (Table 1). Eight weeks of exercise training has been shown to be ineffective in significantly altering blood pressure in humans, indicating our lack of robust MAP changes to be similar to occurrences in a human study (Donley, et al., 2014).

Coat scores and corticosterone concentrations were used to quantify stress in rodents (Figures 2 and 3). Corticosterone ELISA showed LZR C to have markedly decreased corticosterone concentration compared to all other groups. UCMS was effective in increasing corticosterone concentration in LZR animals. Exercise training also increased corticosterone concertation in LZR animals. OZR animals showed a marked increase in corticosterone levels, with UCMS and Ex both increasing corticosterone above control levels. Corticosterone concentrations fluctuate diurnally in rodents, resulting in time of blood draw being extremely important when determining corticosterone levels (Weitzman, et al., 1971). Blood samples were taken at different times throughout the day, making it difficult to rely on corticosterone as an accurate measure of stress.

Coat scores were also used as a marker of stress. Coat score data showed LZR C animals to have markedly better scores than all. UCMS and UCMS+Ex in LZR animals increased coat score values significantly. All OZR coat scores were higher than LZR C, but all other OZR groups showed higher scores than OZR C. In the exercise trained animals this is likely due to lesions suffered from the running protocol, due to their fat 
pads rubbing on the treadmill. When coat score locations OZR animals could not reach (i.e. back and neck) were removed a decrease in coat scores in OZR animals was observed, but scores were still much higher than OZR C. As a result a differentiation should be made in the future between injury and a dirty coat.

Exercise training was able to dramatically improve endothelial-dependent vasoreactivity in the aortas of LZR, OZR, and UCMS animals. Our findings are supported by previous data that exercise improves vasodilation in obese rats and rats exposed to UCMS see detriments to vessel health (Graham, et al., 2003; Delp, et al., 1993; Stanley, et al., 2014). LZR Ex animals saw a marked increase in dilation ability compared to all groups, as did OZR Ex compared to controls. These results exemplify exercise as a great benefit to vascular health. UCMS caused a decrease in methacholine-induced vasodilation, verifying UCMS to be capable of causing endothelial dysfunction and providing further knowledge of the parallels between UCMS and depression in humans.

This study is the first to show exercise as capable of improving endothelial dysfunction caused by MetS and depressive-like symptoms. Both LZR UCMS+Ex and OZR UCMS+Ex groups saw vasodilation return to their respective control values (Figure 11). This finding highlights the potential benefit of exercise training for the vasculature of individuals suffering stress, anxiety, and depression, in addition to the multiple other benefits exercise has been shown to have in the depressed (Moylan, et al., 2013). Exercise training can overcome detriments elicited by UCMS and MetS, suggesting an individual suffering from depression and MetS could see improvements in vascular health, in addition to the mental and overall health benefits of exercise (Teri, et al., 2003; Nabkasorn, et al., 2006). 
Aortic NO bioavailability was also dramatically altered by MetS and the UCMS protocol. OZR C animals showed a significant decrease in NO bioavailability when compared to LZR C animals. In both LZR and OZR animals exposed to UCMS a marked decrease in NO bioavailability was shown. The combination of MetS and UCMS resulted in complete irradiation of NO bioavailability. Exercise training was able to increase NO bioavailability in both LZR and OZR animals, bringing OZR Ex levels to that of LZR C animals. Exercise training was able to rescue NO levels in UCMS animals, notably and significantly improving bioavailability (Figure 12). Our results regarding the ability of exercise to increase NO bioavailability (Graham, et al., 2004; Green, et al., 2004) and for UCMS to decrease NO levels (d'Audiffret, 2010) were in accordance with previous studies. However, this study is the first to show exercise as capable of returning NO bioavailability to basal levels in the presence of UCMS. The increase in NO bioavailability with exercise and decrease with depressive-like symptoms provides rationale for the observations regarding the changes in endothelial-dependent dilation. 
Chapter 5:

Discussion 
The present studies demonstrate the effectiveness of exercise as a treatment for vascular dysfunction, in the conduit arteries, in the presence of MetS, depression, and the two as comorbidities. Our data show the detrimental effects of MetS, chronic stress/depressive-like symptoms, and the comorbid state of these two afflictions on vascular function. Additionally, our data also show the finding that exercise training is capable of stimulating positive vessel remodeling and improving endothelial function and NO bioavailability. Our data also show the protective effect of exercise to be independent of MAP changes. The observation of positive changes to the conduit vessels and NO bioavailability, without significant changes in MAP, leads us to hypothesize the mechanism through which exercise combats vascular dysfunction in MetS and depressive-like symptoms to be one centered on its anti-inflammatory effect.

The UCMS protocol is a verified and acceptable model for inducing depressivelike symptoms in rodents (Willner, 1997). Our data confirm the effectiveness of the UCMS protocol in causing depressive-like symptoms, with stressed animals showing markedly higher coat scores, and verify the UCMS as viable in causing vascular dysfunction (Stanley, et al., 2014). To bolster coat score data, plasma was collected and subjected to an ELISA to determine corticosterone concentration. LZR C animals showed markedly lower corticosterone concentrations than all other groups, which was to be expected. UCMS increased corticosterone concentrations is both LZR and OZR animals (Figure 2). However, exercise training also showed an increase in corticosterone. Cortisol has been shown to increase during exercise training, with more dynamic increases with more intense and longer duration exercise (Kinderman, et al., 1982). However, cortisol has been shown to return to baseline several hours post exercise (Few, 1974). A potential 
answer for this corticosterone conundrum lies within the relationship between cortisol and glucose/insulin. Following a meal cortisol has been shown to increase in circulation, accompanying an increase in glucose metabolism and insulin secretion (Van Cauter, et al., 1992). Our rats were not fasted prior to terminal surgery and blood draw, potentially explaining the unexpected cortisol values in exercise-trained rats. Corticosterone/cortisol is a difficult reading to accurately achieve given its diurnal cycle and release in situations of stress, such as being transported from the animal facility to the laboratory (Putignano, et al., 2001). Given this information we remain confident our use of the UCMS protocol was effective in inducing depressive-like symptoms and exercise did not cause a depressive-like state in our rodents, based on vascular reactivity and remodeling data.

Our results also further confirm the OZR as a viable model for MetS (Frisbee, et al., 2005; de Artinano, et al., 2009). Our finding that MAP did not significantly decrease in most groups with exercise is in conjunction with previous studies utilizing treadmill running and Zucker rats (Peterson, et al., 2008; Frisbee, et al., 2006; Xiang, et al., 2004). The slight decrease in MAP in OZR UCMS+Ex animals could be due to the elevated work the animals performed compared to other exercise groups (Table 1). In addition, the lack of robust changes in LZR UCMS+Ex animals may be explained by the decreased work performed by these animals (Figure 1). Future studies are needed to determine appropriate work load for animals to maximize training effect.

Exercise training was shown to be robustly effective in improving endothelial function and NO bioavailability and moderately effective in structural remodeling changes. These results, paired with our lack of significant findings in MAP, lead us to believe the vascular protective effects of exercise are driven through changes in endothelium function 
and health. As blood pressure is a key determinant of vascular remodeling (Chantler \& Frisbee, 2014) the lack of stark changes in the structural remodeling on the carotid artery (Figures 4-9) is not a surprise. MetS and UCMS did cause deleterious changes the structural remodeling of the carotid, indicating these diseases activate blood pressureindependent pathways, such as inflammation and immune infiltration, to cause hypertrophic remodeling.

Exercise training dramatically improved endothelium-dependent dilation and NO bioavailability in OZR and LZR animals, and was able to recover these indices from the harmful effects of chronic stress (Figures 10-12). Given these robust changes it is clear the protective and beneficial effects of exercise on the conduit arteries, in this model, are an endothelium and NO-driven event. UCMS significantly impacted the ability of the conduit arteries to dilate and produce NO in both LZR and OZR groups (Figures 11-12). This negation of NO-driven events can likely be attributed to a decrease in vascular stem cell proliferation and increases in inflammation/ROS (Kaur, 2014).

In humans, exercise training was not shown to make significant blood pressure changes. However, exercise was shown to improve arterial health (Donley, et al., 2014). Our results further confirm the ability of exercise to improve vascular health, even in the absence of changes in MAP (Table 1). The phenomenon of improvements in vascular health, without improving blood pressure or significantly reducing weight loss, which are key components of MetS, shows exercise to be capable of treating the underlying causes of cardiovascular disease without directly addressing MetS risk factors. It is possible if we extended the duration of our study we would see significant reductions in blood pressure. Additionally, we cannot definitively say we did not improve risk factors associated with 
MetS, as we do not have glucose or lipid data. It is possible our study induced improvements in glucose and lipid metabolism, effectively improving MetS risk factors. Based on our data we feel confident our study demonstrates the ability of exercise training to improve endothelial dysfunction, nitric oxide bioavailability, and structural remodeling in the conduit arteries in healthy controls, rodents with MetS, and rodents going through the UCMS protocol.

\section{Limitations}

Our study was not without limitations. Small sample sizes were present in some carotid remodeling groups. However, we are confident in our results given the replicability of inner and outer diameter values measured among the different animals. Additionally, the minimal variance in the smaller sample groups aided the results in being statistically significant. As previously mentioned the amount of work performed by the exercise training groups was inconsistent. OZR UCMS+Ex animals performed significantly more work than other groups, with LZR UCMS+Ex performing significantly less work. The discrepancy in work between groups may explain the more robust changes in OZR animals than LZR, as the OZR animals performed more work, suggesting their exercise training would be more beneficial in altering the vasculature. However, the vascular health of OZR animals was shown to be far worse than LZR animals, providing rationale that exercise intervention would have a more drastic impact on the health of the vasculature in obese, diseased animals.

Another limitation is the non-differentiating nature of how coat scores were performed. Animals were rated as a " 0 " for clean or "1" for dirty. However, no 
differentiation was made between injury and dirt accumulation associated with lack of grooming. Rodents exposed to UCMS protocol were observed to partake in self-mutilating behavior, through the biting and scratching of different areas of the body. These actions are typically included when coat scoring stressed animals, as they are an indication of stress and depressive-like activities. However, exercise animals also were found to have sores and injuries. These ailments were the result of treadmill running and not self-harm. When coat scoring animals details of treadmill versus self-harm injuries were not considered. As a result, exercise animals likely have higher coat scores than they should. This discrepancy takes away from the level of stress UCMS animals were undergoing. OZR Ex and OZR UCMS+Ex animals had coat scores near OZR UCMS animals. If a differentiation was made it is possible a clearer image of the deleterious effects of chronic stress paired with MetS would be apparent.

A final limitation deals with the metabolic parameters of the study. Animals were not fasted before glucose analyzation, providing an unclear image of basal glucose levels in these animals. Additionally, insulin measurements were performed by clinical laboratories at WVU. Insulin measurements did not yield readable numbers, potentially due to the lower circulating levels of insulin in rat blood. As a result these two important metabolic parameters are not accurately depicted in this experiment. However, we feel confident in stating the OZR is an adequate model of MetS due to its decreased NO bioavailability, negative vascular remodeling, and impaired vasoreactivty to pharmacological agents. 


\section{Future Research}

With these data, and other data affiliated with this study, future research experiments appear promising More extensive laboratory methods are needed to fully understand the molecular events occurring in the conduit arteries. Although western blots were attempted they were not clear enough to present in this thesis. Four targets were selected for protein quantification; MMP-9, SOD-1, TIMP-1, and Collagen-I. MMP-9 was selected due to its role in vascular remodeling, and known relationship with inflammation and vascular injury (Galis and Khatri, 2002). TIMP-1 was selected due to its role in regulating MMPs, and because an imbalance in the two often leads to hypertrophic remodeling (Rodriguez, et al., 2010). Collagen-I was selected as it is pivotal to arterial structure and function, and increase in collagen indicates a stiffer vessel undergoing remodeling. Lastly, SOD-1 was selected due to its known upregulation in exercise and potent ability to scavenge ROS, thereby improving vascular health. (Chantler \& Frisbee, 2014).

Of these targets, westerns for MMP-9 and SOD-1 yielded visible results. However, an inability to develop $\beta$-actin as a control leveled the results unusable. The lack of ability to see $\beta$-actin could stems from a problem with the application of the secondary anti-body, however primary and secondary antibodies were remade multiple times in attempt to troubleshoot. Another potential cause was the amount of times the membranes were stripped before $\beta$-actin administration. Collagen-I has a very high molecular weight, requiring a longer transfer period from gel to membrane. Our inability to quantify collagenI on our membrane is most likely due to an inadequate time spent in transfer (Wang, et al., 2006). Lastly, TIMP-1 requires a lower dilution factor than the other targets assessed 
in this study. TIMP-1 antibody was diluted to a far greater degree than in other literature, resulting in an inability to detect TIMP-1 on our membrane (Wei, et al., 2015).

The kidney presents an interesting role in the development of MetS and depression. Angiotensin II, part of the renin-angiotensin-aldosterone system (RAAS) plays a pivotal role in controlling blood pressure. However, angiotensin II upregulation can result in initiating pathologies such as MetS and become exacerbated by depression. This increase in angiotensin II circulating and binding stimulates inflammation and ROS generation, MMP activation, decreases in eNOS expression, vascular smooth muscle constriction, immune infiltration, and vascular dysfunction. This cascade of events provides rationale for investigating the relationship between renal function and MetS and depressive-like symptoms (Brown, 2013).

Kidney injury molecule-1 (KIM-1) is a relatively newly discovered marker of renal damage, specifically proximal tubular damage (Han, et al., 2002). KIM-1 has been shown to be upregulated in in nearly all proteinuric, toxic, and ischemic kidney diseases providing rationale to study its activity in a variety of diseased states. KIM-1 is expressed early in renal disease, which is often difficult to assess during its beginning stages (Waanders, et al., 2010). As such, KIM-1 may be a potential target for understanding and treating endstage renal disease (ESRD), which commonly accompanies MetS and diseases of the CV system. The relationship between exercise and KIM-1 has not been adequately studied, however exercise has been shown to beneficial in the rehabilitation of individuals suffering from ESRD (Painter, 1994). 


\section{Conclusions}

Exercise training was shown to be effective in reducing the vascular dysfunction associated with MetS and depressive-like symptoms. Exercise was able to marginally improve functional remodeling in the carotid artery and was shown to have dynamic effects on endothelial function and NO bioavailability in the aorta, suggesting the beneficial effects of exercise to be more prevalent in the endothelium than in altering the structural components of the arterial wall. 
Acknowledgments

This study was supported by American Heart Association Grants IRG 14330015, PRE 16850005, and EIA 0740129N and National Institutes of Health Grants 1P20 GM-109098, RR-2865AR, and P20 RR-016477. This research was supported by the National Institute of General Medical Sciences Grant U54 GM-104942. 


\section{References}

Bouvet, C., Belin de Chantemele, E., Guihot, A. L., Vessieres, E., Bocquet, A., Dumont, O., et al. (2007). Flow-induced remodeling in resistance arteries from obese zucker rats is associated with endothelial dysfunction. Hypertension, 50(1), 248-254. doi:HYPERTENSIONAHA.107.088716 [pii]

Brown, N. J. (2013). Contribution of aldosterone to cardiovascular and renal inflammation and fibrosis. Nature Reviews Nephrology, 9(8), 459-469.

Cacicedo, J. M., Gauthier, M. S., Lebrasseur, N. K., Jasuja, R., Ruderman, N. B., \& Ido, Y. (2011). Acute exercise activates AMPK and eNOS in the mouse aorta. American Journal of Physiology.Heart and Circulatory Physiology, 301(4), H1255-65. doi:10.1152/ajpheart.01279.2010 [doi]

d'Audiffret, A. C., Frisbee, S. J., Stapleton, P. A., Goodwill, A. G., Isingrini, E., \& Frisbee, J. C. (2010). Depressive behavior and vascular dysfunction: A link between clinical depression and vascular disease? Journal of Applied Physiology (Bethesda, Md: 1985), 108(5), 1041-1051. doi:10.1152/japplphysiol.01440.2009 [doi]

de Artiñano, A. A., \& Castro, M. M. (2009). Experimental rat models to study the metabolic syndrome. British Journal of Nutrition, 102(09), 1246-1253.

Delp, M. D., McAllister, R. M., \& Laughlin, M. H. (1993). Exercise training alters endothelium-dependent vasoreactivity of rat abdominal aorta. Journal of Applied Physiology (Bethesda, Md.: 1985), 75(3), 1354-1363.

Donley, D. A., Fournier, S. B., Reger, B. L., DeVallance, E., Bonner, D. E., Olfert, I. M., et al. (2014). Aerobic exercise training reduces arterial stiffness in metabolic syndrome. Journal of Applied Physiology (Bethesda, Md.: 1985), 116(11), 13961404. doi:10.1152/japplphysiol.00151.2014 [doi]

Dunbar, J. A., Reddy, P., Davis-Lameloise, N., Philpot, B., Laatikainen, T., Kilkkinen, A., et al. (2008). Depression: An important comorbidity with metabolic syndrome in a general population. Diabetes Care, 31(12), 2368-2373. doi:10.2337/dc08-0175 [doi]

Few, J. D. (1974). Effect of exercise on the secretion and metabolism of cortisol in man. The Journal of Endocrinology, 62(2), 341-353.

FRISBEE, J. C. (2005). Hypertension-independent microvascular rarefaction in the obese zucker rat model of the metabolic syndrome. Microcirculation, 12(5), 383-392.

Frisbee, J. C., Samora, J. B., Peterson, J., \& Bryner, R. (2006). Exercise training blunts microvascular rarefaction in the metabolic syndrome. American Journal of Physiology. Heart and Circulatory Physiology, 291(5), H2483-92. doi:00566.2006 [pii] 
Galis, Z. S., \& Khatri, J. J. (2002). Matrix metalloproteinases in vascular remodeling and atherogenesis: The good, the bad, and the ugly. Circulation Research, 90(3), 251262.

Graham, D. A., \& Rush, J. W. (2004). Exercise training improves aortic endotheliumdependent vasorelaxation and determinants of nitric oxide bioavailability in spontaneously hypertensive rats. Journal of Applied Physiology (Bethesda, Md.: 1985), 96(6), 2088-2096. doi:10.1152/japplphysiol.01252.2003 [doi]

Green, D. J., Maiorana, A., O'Driscoll, G., \& Taylor, R. (2004). Effect of exercise training on endothelium-derived nitric oxide function in humans. The Journal of Physiology, 561(1), 1-25.

Heinonen, I., Rinne, P., Ruohonen, S., Ruohonen, S., Ahotupa, M., \& Savontaus, E. (2014). The effects of equal caloric high fat and western diet on metabolic syndrome, oxidative stress and vascular endothelial function in mice. Acta Physiologica, 211(3), 515-527.

Intengan, H. D., \& Schiffrin, E. L. (2001). Vascular remodeling in hypertension: Roles of apoptosis, inflammation, and fibrosis. Hypertension, 38(3 Pt 2), 581-587.

Kindermann, W., Schnabel, A., Schmitt, W., Biro, G., Cassens, J., \& Weber, F. (1982). Catecholamines, growth hormone, cortisol, insulin, and sex hormones in anaerobic and aerobic exercise. European Journal of Applied Physiology and Occupational Physiology, 49(3), 389-399.

Kiuchi, T., Lee, H., \& Mikami, T. (2012). Regular exercise cures depression-like behavior via VEGF-flk-1 signaling in chronically stressed mice. Neuroscience, 207, 208-217.

Olijhoek, J. K., van der Graaf, Y., Banga, J. D., Algra, A., Rabelink, T. J., Visseren, F. L., et al. (2004). The metabolic syndrome is associated with advanced vascular damage in patients with coronary heart disease, stroke, peripheral arterial disease or abdominal aortic aneurysm. European Heart Journal, 25(4), 342-348. doi:10.1016/j.ehj.2003.12.007 [doi]

Painter, P. (1994). The importance of exercise training in rehabilitation of patients with end-stage renal disease. American Journal of Kidney Diseases : The Official Journal of the National Kidney Foundation, 24(1 Suppl 1), S2-9; discussion S31-2. doi:S0272638694001447 [pii]

Pattyn, N., Cornelissen, V. A., Eshghi, S. R. T., \& Vanhees, L. (2013). The effect of exercise on the cardiovascular risk factors constituting the metabolic syndrome. Sports Medicine, 43(2), 121-133.

Peterson, J. M., Bryner, R. W., Sindler, A., Frisbee, J. C., \& Alway, S. E. (2008). Mitochondrial apoptotic signaling is elevated in cardiac but not skeletal muscle in the 
obese zucker rat and is reduced with aerobic exercise. Journal of Applied Physiology (Bethesda, Md.: 1985), 105(6), 1934-1943. doi:10.1152/japplphysiol.00037.2008 [doi]

Pinheiro, A. R., Cunha, A. R., Aguila, M. B., \& Mandarim-de-Lacerda, C. A. (2007). Beneficial effects of physical exercise on hypertension and cardiovascular adverse remodeling of diet-induced obese rats. Nutrition, Metabolism and Cardiovascular Diseases, 17(5), 365-375.

Putignano, P., Dubini, A., Toja, P., Invitti, C., Bonfanti, S., Redaelli, G., ... \& Cavagnini, F. (2001). Salivary cortisol measurement in normal-weight, obese and anorexic women: comparison with plasma cortisol. European Journal of Endocrinology, 145(2), 165-171.

Rodríguez, D., Morrison, C. J., \& Overall, C. M. (2010). Matrix metalloproteinases: what do they not do? New substrates and biological roles identified by murine models and proteomics. Biochimica et Biophysica Acta (BBA)-Molecular Cell Research, 1803(1), 39-54.

Schillaci, G., Pirro, M., Vaudo, G., Mannarino, M. R., Savarese, G., Pucci, G., et al. (2005). Metabolic syndrome is associated with aortic stiffness in untreated essential hypertension. Hypertension, 45(6), 1078-1082. doi:01.HYP.0000165313.84007.7d [pii]

Scuteri, A., Naijar, S. S., Muller, D. C., Andres, R., Hougaku, H., Metter, E. J., et al. (2004). Metabolic syndrome amplifies the age-associated increases in vascular thickness and stiffness. Journal of the American College of Cardiology, 43(8), 1388-1395.

Seldenrijk, A., van Hout, H. P., van Marwijk, H. W., de Groot, E., Gort, J., Rustemeijer, C., et al. (2011). Depression, anxiety, and arterial stiffness. Biological Psychiatry, 69(8), 795-803.

Shimbo, D., Chaplin, W., Crossman, D., Haas, D., \& Davidson, K. W. (2005). Role of depression and inflammation in incident coronary heart disease events. The American Journal of Cardiology, 96(7), 1016-1021.

Stanley, S. C., Brooks, S. D., Butcher, J. T., d'Audiffret, A. C., Frisbee, S. J., \& Frisbee, J. C. (2014). Protective effect of sex on chronic stress- and depressive behaviorinduced vascular dysfunction in BALB/cJ mice. Journal of Applied Physiology (Bethesda, Md.: 1985), 117(9), 959-970. doi:10.1152/japplphysiol.00537.2014 [doi]

Stepp, D. W., Pollock, D. M., \& Frisbee, J. C. (2004). Low-flow vascular remodeling in the metabolic syndrome X. American Journal of Physiology.Heart and Circulatory Physiology, 286(3), H964-70. doi:10.1152/ajpheart.00836.2003 [doi] 
Tanaka, H., Seals, D. R., Monahan, K. D., Clevenger, C. M., DeSouza, C. A., \& Dinenno, F. A. (2002). Regular aerobic exercise and the age-related increase in carotid artery intima-media thickness in healthy men. Journal of Applied Physiology, 92(4), 1458-1464.

Van Cauter, E., Shapiro, E. T., Tillil, H., \& Polonsky, K. S. (1992). Circadian modulation of glucose and insulin responses to meals: Relationship to cortisol rhythm. The American Journal of Physiology, 262(4 Pt 1), E467-75.

Waanders, F., van Timmeren, M. M., Stegeman, C. A., Bakker, S. J., \& van Goor, H. (2010). Kidney injury molecule-1 in renal disease. The Journal of Pathology, 220(1), 7-16.

Wang, X., LeMaire, S. A., Chen, L., Shen, Y. H., Gan, Y., Bartsch, H., ... \& Wang, X. L. (2006). Increased collagen deposition and elevated expression of connective tissue growth factor in human thoracic aortic dissection. Circulation, 114(1 suppl), I-200.

Wei, H., Wang, S., Zhen, L., Yang, Q., Wu, Z., Lei, X., ... \& Xue, R. (2015). Resveratrol attenuates the blood-brain barrier dysfunction by regulation of the MMP-9/TIMP-1 balance after cerebral ischemia reperfusion in rats. Journal of Molecular Neuroscience, 55(4), 872-879.

Willner, P. (1997). Validity, reliability and utility of the chronic mild stress model of depression: A 10-year review and evaluation. Psychopharmacology, 134(4), 319329.

Xiang, L., Naik, J., \& Hester, R. L. (2005). Exercise-induced increase in skeletal muscle vasodilatory responses in obese zucker rats. American Journal of Physiology.Regulatory, Integrative and Comparative Physiology, 288(4), R987-91. doi:00702.2004 [pii]

Yaffe, K., Kanaya, A., Lindquist, K., Simonsick, E. M., Harris, T., Shorr, R. I., et al. (2004). The metabolic syndrome, inflammation, and risk of cognitive decline. Jama, 292(18), 2237-2242.

Zucker, L. M., \& Antoniades, H. N. (1972). Insulin and obesity in the zucker genetically obese rat "fatty". Endocrinology, 90(5), 1320-1330. 